\title{
DESIGNING A ROBUST SUPPLY CHAIN NETWORK AGAINST DISRUPTIONS
}

\author{
A Dissertation \\ presented to \\ the Faculty of the Graduate School \\ at the University of Missouri-Columbia
}

In Partial Fulfillment

of the Requirements for the Degree

Doctor of Philosophy

by
MAHMOOD PARIAZAR

Dr. Mustafa Y. Sir, Dissertation Supervisor

MAY 2013 
(C) Copyright by Mahmood Pariazar 2013

All Rights Reserved 
The undersigned, appointed by the Dean of the Graduate School, have examined the dissertation entitled:

\section{DESIGNING A ROBUST SUPPLY CHAIN NETWORK AGAINST DISRUPTIONS}

presented by Mahmood Pariazar, a candidate for the degree of doctor of philosophy and hereby certify that, in their opinion, it is worthy of acceptance.

Dr. Mustafa Y. Sir

Dr. Esra Sisikoglu

Dr. Luis Occena

Dr. James Noble

Dr. Timothy C. Matisziw

Dr. Sarah Root 


\section{DEDICATION}

This dissertation is dedicated to my wife, Shaghayegh Parhiz, for all of her support, inspiration, and love. 


\section{ACKNOWLEDGMENTS}

I would like to express my deepest appreciation toward my family, professors, colleagues, and friends for their persistent help and encouragement.

First of all, I would like to express the deepest gratitude to my committee chair Dr. Sir, who provided me guidance and insights to break through the problems. This dissertation would not have been possible without his advice, help, and support. Getting an opportunity to work with Dr. Sir, was an honor and a privilege indeed.

Also, I would like to acknowledge with much appreciation the crucial role of Dr. Sisikoglu and Dr. Root for their inputs and useful comments on this dissertation. Furthermore, I would like to gratefully and sincerely thank my other committee members, Dr. Occena, Dr. Nobel, and Dr. Matisziw for their useful comments, remarks and engagement on my dissertation.

Further, I would also like to thank all my instructors and teachers specially Dr. Sir, Dr. Chang, Dr. Jang, Dr. Occena, and Dr Sisikoglu for their support and encouragement and invaluable guidance over the past 4 years.

In addition, I would like to thank "Center for Excellence in Logistics and Distribution (CELDi)" which provided me the opportunity to work on three supply chain projects namely "Ensuring continuity of care: A quantification of risk in the healthcare supply chain, sponsored by National Science Foundation, Grant IIP-0815195" and "Sign production and inventory logistics study, sponsored by Missouri Department of Transportation, award \# TRyy1122", and "Bayer on demand logistics network design, sponsored by Bayer CropScience, award \#0033623”.

Last but not least, my very special thanks to my parents for their unconditional love, support, and faith in my abilities. 


\section{TABLE OF CONTENTS}

ACKNOWLEDGMENTS ................ ii

LIST OF FIGURES $\ldots \ldots \ldots \ldots \ldots \ldots$ vi

LIST OF TABLES $\ldots \ldots \ldots \ldots \ldots \ldots$ viii

ABSTRACT ...................

\section{CHAPTER}

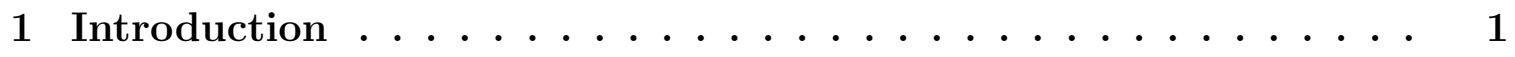

1.1 Significance and Motivation _. . . . . . . . . . . . . 1

1.2 Specific Aims $\ldots \ldots \ldots \ldots \ldots \ldots \ldots \ldots \ldots$

1.3 Innovation $\ldots \ldots \ldots \ldots \ldots \ldots \ldots \ldots \ldots \ldots \ldots$

2 Robust supply chain design considering correlated failures and inspection .................... 9

2.1 Introduction and motivation $\ldots \ldots \ldots \ldots \ldots$

2.2 Model . . . . . . . . . . . . . . . . . . . . 17

2.2 .1 Sets and Parameters . . . . . . . . . . . . . . 20

2.2.2 Stochastic Programming Formulation . . . . . . . . . . . 21

2.2.3 Scenario Generation for Correlated Facility Failures . . . . . 25

2.2.4 Solution Methodology . . . . . . . . . . . . . . . . 30

2.3 Computational Experiments . . . . . . . . . . . . . . 30

2.3 .1 Effect of Capacity . . . . . . . . . . . . . . . . . 36

2.3.2 Effect of Correlation . . . . . . . . . . . . . . 37

2.3 .3 Effect of Inspection . . . . . . . . . . . . . . . . . . 39

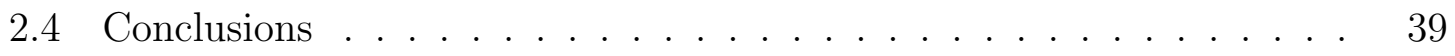


3 A multi-objective approach for designing a robust supply chain .

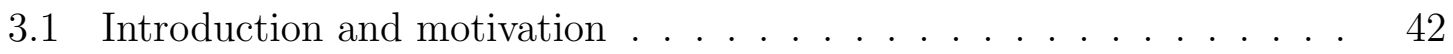

3.2 A Multi-objective supply chain model . . . . . . . . . . . . . 51

3.2.1 Mathematical formulation of the model: . . . . . . . . . 54

3.2 .2 Disruption scenario generation $\ldots \ldots \ldots \ldots \ldots$

3.3 Solution approach . . . . . . . . . . . . . . . . . . . . . . . . 59

3.3.1 Data Envelopment Analysis. . . . . . . . . . . . . . . . . . 59

3.3 .2 Hybrid Genetic Algorithm. . . . . . . . . . . . . . . . 60

3.4 Numerical Experiments . . . . . . . . . . . . . . . . . . . . 65

3.4.1 An example of a medium scale problem . . . . . . . . . 66

3.4.2 An example of a large scale problem . . . . . . . . . 75

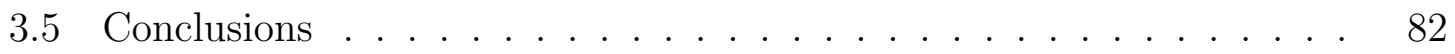

4 A Dynamic programming approach for optimal inspection scheduling ...................... 84

4.1 Introduction and motivation . . . . . . . . . . . . . 84

4.1 .1 Literature review . . . . . . . . . . . . . . . 86

4.2 Problem description and notation $\ldots \ldots \ldots \ldots$

4.2.1 Dynamic Programming Formulation . . . . . . . . . . . . 92

4.3 Single supplier . . . . . . . . . . . . . . . . . . . . . . . . . . . 98

4.4 Linear programming formulation of DP model . . . . . . . . . 107

4.5 An approximate dynamic programming model . . . . . . . . . 108

4.5.1 Action reduction-Constraints reduction . . . . . . . . . . 109

4.5.2 State reduction-Variables reduction . . . . . . . . . . 111

4.5.3 Extreme states method - State and action reduction . . . . . . 114

4.5.4 An extended of the Extreme-states method . . . . . . . . . 116 
4.6 Numerical Results . . . . . . . . . . . . . . . . . . . . . . . . . 117

4.7 Conclusions . . . . . . . . . . . . . . . . . . . 123

5 Summary and concluding remarks . . . . . . . . . . . 124

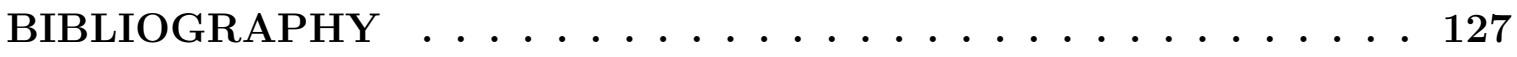

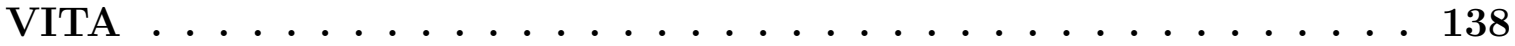




\section{LIST OF FIGURES}

Figure

2.1 Two-tier supply supply chain. . . . . . . . . . . . . . . . 27

2.2 Outline of scenario generation procedure for correlated facility failures. 29

2.3 Candidate facility locations, raw material supplier locations, and cus-

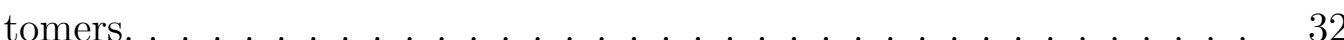

2.4 Effect of capacity on (a) expected total cost and (b) expected cost of shipped tainted materials. Radius determines the level of correlation: radius $=5$ represents no correlation, radius $=9$ medium correlation, and radius $=12$ high correlation. In the experiments, the marginal mean of failure is 0.02 for all facilities. . . . . . . . . . . . .

2.5 Effect of correlation on (a) expected total cost and (b) expected cost of shipped tainted materials. Radius determines the level of correlation: radius $=5$ represents no correlation, radius $=9$ medium correlation, and radius $=12$ high correlation. In the experiments, the marginal mean vector of facility failures is set to $\left[\begin{array}{llllll}0.025 & 0.02 & 0.015 & 0.06 & 0.05 & 0.015\end{array}\right] .38$

2.6 Effect of inspection option on (a) expected total cost and (b) expected cost of shipped tainted materials. In the experiments, radius of facility circles is set to 9 and the marginal mean vector of facility failures is set to $\left[\begin{array}{llllll}0.025 & 0.02 & 0.015 & 0.06 & 0.05 & 0.015\end{array}\right]$ while capacity is varied. . . . 40 
2.7 Effect of inspection on (a) expected total cost and (b) expected cost of shipped tainted materials. In the experiments, capacity is set to $30 \%$ more than the total demand and the marginal mean vector of

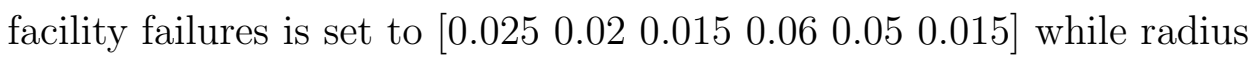
of facility circles is varied. . . . . . . . . . . . . . 40

3.1 Framework for hybrid genetic algorithm. . . . . . . . . . . . 61

3.2 An example of chromosome representation . . . . . . . . . . 61

3.3 An example of the replacement operation. . . . . . . . . . . 65

3.4 Potential facilities and raw material suppliers $\ldots \ldots \ldots \ldots$

3.5 Two by two comparison between $g_{1}, g_{2}, g_{3}, \theta \ldots \ldots \ldots \ldots$

3.6 Comparison between two approaches $\ldots \ldots \ldots \ldots \ldots$

3.7 GA results for a given population number, crossover rate, and mutation rate where required iterations are equal to $50 \ldots \ldots \ldots 78$

3.8 GA results for a given population number, crossover rate, and mutation rate where required iterations are equal to $100 \ldots \ldots \ldots$. . . . 79

3.9 Reliable vs. unreliable solution _ . . . . . . . . . . . . . . . . 82

4.1 Supply Inspection $\ldots \ldots \ldots$

4.2 Markov process for a given supplier without inspection . . . . . . 90

4.3 Decision tree (IC: in compliance, OC: out of compliance) $\ldots \ldots \ldots 91$

4.4 State transition diagrams . . . . . . . . . . . . . . . . . 96

4.5 An example of threshold policy for a single-supplier (DI: Do not inspect, I: Inspect $) \quad \ldots \ldots \ldots \ldots$ 


\section{LIST OF TABLES}

Table Page

1.1 Summary of proposed chapters $\ldots \ldots \ldots \ldots \ldots$

2.1 Example of a two-tier supply chain. . . . . . . . . . . . . 28

2.2 Correlation matrix of the two-tier supply chain in Table $\ldots \ldots .28$

2.3 Candidate facility locations. . . . . . . . . . . . . . . . 31

2.4 Correlation matrix $($ radius $=5) \ldots \ldots \ldots \ldots \ldots \ldots$

2.5 Correlation matrix $($ radius $=9) \ldots \ldots \ldots \ldots \ldots \ldots \ldots$

2.6 Correlation matrix $($ radius $=12) \ldots \ldots \ldots \ldots \ldots \ldots$

2.7 Cases for marginal means of facility failures. . . . . . . . . . . 34

2.8 Facility Capacities. . . . . . . . . . . . . . . . . . . . . . . 34

3.1 Potential facilities . . . . . . . . . . . . . . . . . 66

3.2 Correlation matrix $\ldots \ldots \ldots \ldots \ldots$

3.3 Some examples of possible solutions . . . . . . . . . . 70

3.4 GA parameters . . . . . . . . . . . . . . 73

3.5 Potential facilities . . . . . . . . . . . . . . . . 75

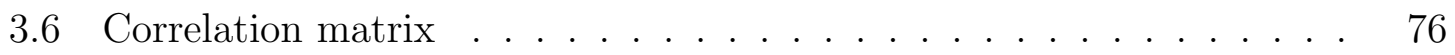

3.7 GA parameters $\ldots \ldots \ldots \ldots \ldots \ldots \ldots \ldots \ldots \ldots$

3.8 Extreme results $\ldots \ldots \ldots \ldots \ldots$

3.9 Reliable vs. unreliable solution $\ldots \ldots \ldots \ldots \ldots$

4.1 Possible states and actions for single supplier . . . . . . . . . 107

4.2 Problem size for a given $k, \rho \ldots \ldots \ldots \ldots \ldots \ldots$

4.3 Action reduction process $\ldots \ldots \ldots \ldots$ 
4.4 Dual and Slack values in small example . . . . . . . . . . . . . . . . 116

4.5 Exact method vs. Approximation method . . . . . . . . . . . . 118

4.6 Comparison between approximation approaches changing $\bar{s}$. . . . . . 119

4.7 Comparison between approximation approaches changing $r$. . . . 120

4.8 Comparison between approximation approaches changing $h$. . . . . . 120

4.9 Simulation results: Approximation method vs. Baseline policy . . . . 122 


\title{
DESIGNING A ROBUST SUPPLY CHAIN NETWORK AGAINST DISRUPTIONS \\ Mahmood Pariazar \\ Dr. Mustafa Y. Sir, Dissertation Supervisor
}

\begin{abstract}
Supply chains are vulnerable to disruptions at any stage of the distribution system. These disruptions can be caused by natural disasters, production problems, or labor defects. The consequences of these disruptions may result in significant economic losses or even human deaths. Therefore, it is important to consider any disruption as an important factor in strategic supply chain design. Consequently, the primary outputs of this dissertation include insights for designing robust supply chains that are neither significantly nor adversely impacted by disruptions.

The impact of correlated supplier failures is examined and how this problem can be modeled as a variant of a facility location problem is described. Two main problems are defined, the first being the design of a robust supply chain, and the second being the optimization of operational inspection schedules to maintain the quality of an already established supply chain. In this regard, both strategic and operational decisions are considered in the model and (1) a two-stage stochastic programming model; (2) a multi-objective stochastic programming model; and (3) a dynamic programming model are developed to explore the tradeoffs between cost and risk.

Three methods are developed to identify optimal and robust solutions: an integer L-shaped method; a hybrid genetic algorithm using Data Envelopment Analysis; and an approximate dynamic programming method. Several sensitivity analyses are performed on the model to see how the model output would be affected by uncertainty.

The findings from this dissertation will be able to help both practitioners designing supply chains, as well as policy makers who need to understand the impact of different disruption mitigation strategies on cost and risk in the supply chain.
\end{abstract}




\section{CHAPTER 1. INTRODUCTION}

\subsection{Significance and Motivation}

A supply chain is a network of suppliers, production centers, warehouses, and distribution centers. It is through supply chains that raw materials are acquired, transformed,

produced, and delivered to the customer [1]. The performance of a supply chain is dependent on the performance of each component in the network, and the ability of those components to be organized and integrated.

Due to the complicated interrelations and interactions between a highly diverse set of suppliers, production centers, distribution centers, and customers, supply chains are subject to variety of risk factors. Coordination between those entities is typically an important and difficult activity. A relatively small disruption and failure in one component can lead to a massive negative impact across the entire network.

A disaster is an unforeseen and often sudden event that brings about damage, loss, and destruction to life and property. Common causes of supply chain disruption include natural disasters, production problems, accidents, labor availability, terrorist attacks, unexpected and sudden shocks, political/economic disasters, and war.

In this dissertation, two types of supply chain disruptions are considered. The first type of disruption is one that leads to a quality problem with the product. Failure in this context is a function of low-quality raw materials that a facility receives and consequently that facility will produce some materials that are tainted. The second type of disruption is one that affects the quantity (availability) of the product.

For example, in Japan, the March 11, 2011 earthquake (magnitude 9.0) and subsequent tsunami, nuclear crisis, and infrastructure damage created a significant influence on many companies. Disrupted supply chains affected a broad range of manufacturing industries such as car manufacturing, consumer electronics, and data process- 
ing. These disrupted industries were located not just within Japan but also around the world. Automotive companies such as Honda, Toyota, and Nissan had to shut down production upon being faced with lack of component availability [2, 3]. Clearly, lack of necessary materials creates serious issues for global manufacturing.

Another example: Heparin, a widely used anticoagulant, is produced from the mucosal tissue of pigs. In 2008, when blue ear pig disease swept through China, tainted heparin was produced and distributed [4]. Contaminated heparin caused 81 deaths and several allergic reactions in the United States and around the world. Thereafter, further testing and product recalls of heparin have occurred on order of the U.S. Food and Drug Administration (FDA) [5].

These examples indicate the danger of failures in supply chains. These examples also point to the importance of considering risk in supply chain design. Disruption in supply chains creates significant risk with life and death implications. For example, distributing tainted drugs and/or the lack of availability of drugs can have significant impact on patients' health, a circumstance that can lead to death.

One of the issues raised by the examples is that of correlated supplier failure. These failures are not independent. Such correlation in supply failures can occur for different reasons, including inclement weather, political unrest, or labor issues such as strikes affecting multiple facilities. As a result, it is critical to consider the correlation between suppliers when considering their failure probabilities, since supplier failures are often not independent - as is typically assumed in this type of research.

Moreover, in some supply chains, inventory shortages and unavailability of products are as problematic as the shipment of low quality products. For example, unavailability of heparin can cause serious complications for surgery patients since substitutions for this drug cannot be easily made. Therefore, care should be used when selecting suppliers, so that products are available even in the face of failures and disruptions. 
Furthermore, although the suppliers typically produce high-quality products, they can fail and produce (at least partially) a low-quality product. Such failures require the building-in of redundant capacity that can be used to obtain quality product in the face of supplier failures. Another issue raised by these supply chain disruptions is that of supplier inspection. Inspection can be an effective method to ensure that only high-quality products reach customers when a disruption occurs.

Considering all the points mentioned above, this dissertation studies how supply chains should be designed to mitigate the effects of supplier failures, and explores how integrating inspection policy decisions into facility location and supply chain design can impact the solutions that are recommended.

In general, in addressing the risk of supplier unreliability in the supply chain, three optimization models and algorithms are developed in order to:

- explore the tradeoffs between costs and risk in the supply chain;

- investigate the effect of correlated failures in a supply chain;

- consider different failure scenarios and examine their effects on inspection strategies;

- develop insights into when/where redundant capacity should be installed in the supply chain;

- incorporate the ability to inspect the goods that are produced from failed locations to see if they can be shipped to the customer;

- understand when and where inspections should be performed to prevent lowquality product from reaching customers;

- study various supply chain configurations resulting from these models on the overall performance of the supply chain; 
- investigate solution methods for this class of computationally challenging largescale problems.

The findings from this dissertation can help practitioners while designing supply chains, as well as helping policymakers to understand the impact of different disruption mitigation strategies on cost and risk in the supply chain.

Table 1.1 represents a summary of proposed chapters considering decision, problem, model, and solution approaches in each chapter.

Table 1.1: Summary of proposed chapters

\begin{tabular}{|c|c|c|c|c|}
\hline Chapter & Decision & Problem & Model & Solution approach \\
\hline $\begin{array}{c}\text { Chapter } \\
2\end{array}$ & $\begin{array}{c}\text { Long-term } \\
\text { (Strategic) }\end{array}$ & $\begin{array}{c}\text { Designing a } \\
\text { robust supply } \\
\text { chain }\end{array}$ & $\begin{array}{c}\text { Two-stage } \\
\text { stochastic } \\
\text { programming }\end{array}$ & $\begin{array}{c}\text { Integer } \\
\text { L-Shaped } \\
\text { method }\end{array}$ \\
\hline $\begin{array}{c}\text { Chapter } \\
3\end{array}$ & $\begin{array}{c}\text { Long-term } \\
\text { (Strategic) }\end{array}$ & $\begin{array}{c}\text { Designing a } \\
\text { robust supply } \\
\text { chain }\end{array}$ & $\begin{array}{c}\text { Multi-objective } \\
\text { stochastic } \\
\text { programming }\end{array}$ & $\begin{array}{c}\text { Hybrid } \\
\text { genetic } \\
\text { algorithm }\end{array}$ \\
\hline $\begin{array}{c}\text { Chapter } \\
4\end{array}$ & Short-term & $\begin{array}{c}\text { Inspection } \\
\text { (Operational) } \\
\text { decision }\end{array}$ & $\begin{array}{c}\text { Dynamic } \\
\text { programming } \\
\text { model }\end{array}$ & $\begin{array}{c}\text { Approximate } \\
\text { DP } \\
\text { algorithm }\end{array}$ \\
\hline
\end{tabular}

In Chapter 2 and Chapter 3 of this dissertation, a supply chain that is both reliable and cost-efficient is designed. Designing a robust supply is a long-term strategic decision and cannot change quickly due to the influence of supply chain infrastructure. Supplier sourcing is considered in two-tiered supply chains. First-tier suppliers are those who produce a product from raw materials or sub-assemblies. Second-tier suppliers harvest or produce the raw materials or sub-assemblies that are ultimately shipped to the first-tier suppliers. It is assumed that customers must partner with one or more first-tier suppliers to obtain the required demand for quality products. Therefore, "opening a facility" in the models is equivalent to entering a long-term contractual agreement with a first-tier supplier. The expected costs are minimized considering disruption scenarios. Operational decisions, such as optimizing the inspection schedules, are considered in the model as well. Both strategic supply chain 
design and operational inspection scheduling are important to ensure availability and quality in supply chains in order to mitigate disruption impacts. A two-stage stochastic programming model is developed and solved with integer L-Shaped method and a hybrid genetic algorithm.

In Chapter 4, the operational inspection decision is examined for a given supply chain with multiple unreliable supply sources. Disruption directly affects the quality

of products. The quality of products can be improved by considering inspection of the units received from the suppliers. Even for a robust supply chain, it is essential to optimize inspection decisions to ensure quality. A dynamic programming model is developed to indicate which set of suppliers need inspection at various periods, according to the various cost terms. An approximate DP algorithm is developed to solve the model.

\subsection{Specific Aims}

In the following parts, goals or aims, are explained for each chapter.

Aim 1: Robust supply chain design considering correlated failures and inspection. In Chapter 2, a robust supply chain design is studied considering unreliable suppliers, correlated failures, and the option to inspect. A comprehensive two-stage stochastic programming model is developed to explore the tradeoffs between costs and risk when designing a supply chain. The first-stage decisions represent strategic decisions such as location and capacity of suppliers. In the second stage, operational decisions related to transportation and inspection are determined. To determine an optimal solution, the integer L-shaped method is used and a sensitivity analysis is applied to the model to see how the model output is affected by uncertainty.

Aim 2: A multi-objective approach for designing a robust supply. In Chapter 3, the single objective version of the problem is extended which is described in Chapter 2, and a multi-objective stochastic model is developed to explore the 
tradeoffs between costs and risk. "Operating cost", "unsatisfied cost", and "tainted and inspection cost" are explicitly considered as three conflicting goals that must be minimized simultaneously. The first objective (operational cost) minimizes the supply chain configuration cost under normal circumstances without disruptions or failures. The second and third objective attempt to minimize the effect of disruption in quantity and quality aspect.

It is important to consider these objectives simultaneously to gain a better understanding of disruption in the supply chain. Since this is a NP-hard problem, multiple Pareto-optimal solutions are generated by using the genetic algorithm, in which data envelopment analysis is used to calculate the fitness value of supply chain configurations. The hybrid approach removes dominated individuals and yields to desirable efficient frontiers.

\section{Aim 3: A Dynamic programming approach for optimal inspection} scheduling. In Chapter 4, operational inspection decisions are investigated in multiperiod supply chain with multiple unreliable supply sources. Even if the supply chain design is robust, it is still important to inspect suppliers to ensure quality. Therefore, Inspection of suppliers is critical to ensure that only high-quality products reach customers. Also, by preventing potential failure, the disruption cost would be cut out. FDA requires inspection every two years. This may not be the most effective and efficient policy because of the limited number of inspection resources and differences in supplier characteristics.

A dynamic programming (DP) model is performed to minimize the total inspection and corrective costs as well as the cost of disruption caused by missed detection in the supply chain. The current FDA inspection policy is evaluated; numerical experiments indicate that proposed DP models result in significantly lower costs than the FDA's current inspection policy. 


\subsection{Innovation}

In this dissertation, an extension of the capacitated facility location problem is proposed that considers correlated supplier failures and integrates the operational decision of whether or not to inspect suppliers while making the long-term decision of which supplier should be selected. This adds to the growing body of literature that integrates tactical-level decisions when making strategic-level facility location decisions. Furthermore, the operational inspection decision is studied for a given supply chain with multiple unreliable supply sources. This dissertation makes a number of contributions to the literature.

First, it relaxes the assumption traditionally made that the failure of suppliers is independent. In practice, this is often not the case, as illustrated by the heparin example above. It is explained how correlated failures can be incorporated into the model via the generation of scenarios that are used in the stochastic program. The computational results show that this has a significant impact both on the cost of the obtained solutions, and the solutions themselves.

Second, this dissertation integrates the strategic decisions of where facilities should be opened with operational level decisions of whether or not inspection should be instituted at each facility.

Third, the assumption that supplier failures are absolute is relaxed - that is, partial failures of suppliers are allowed in the model (i.e., some of the supplies produced from a facility are damaged whereas others are not). This is an important consideration when the underlying cause of failures is due to the second-tier suppliers. In this case, the quality problems may or may not appear in all of the first-tier suppliers depending on their quality practices and the second-tier suppliers with whom they work.

Fourth, a comprehensive two-stage stochastic program is developed. This adds to the growing body of research on robust facility location by considering correlated 
scenarios and incorporating inspection decisions in the second stage. This model is exercised to provide insights about how key parameters (capacity, correlation, inspection) impact the obtained solutions and the expected total cost of solutions.

Fifth, a multi-objective stochastic model is developed to explore the tradeoffs between cost and risk in the supply chain when a disruption occurs.

Sixth, two types of disruption are considered in the model. The first type of disruption is one that leads to a quality problem of the product (low-quality raw materials that a facility receives). This helps us to develop insights about tainted cost and inspection policy. The quality failures are simulated by considering the supplier's correlation. The disruptions that affect the quantity (availability) of the product are considered to be the second type of disruption. This helps us to develop insights about capacity and unsatisfied demand.

Seventh, a hybrid genetic algorithm is developed to solve the multi-objective stochastic model. In this algorithm, data envelopment analysis is used to calculate the fitness value of supply chain configurations. The hybrid approach removes dominating individuals and yields to desirable efficient frontiers faster than methods based on only genetic algorithm.

Eighth, in the last chapter, the operational inspection decisions are examined for a given supply chain. However, the same concept can be used in a variety of application areas such as machine replacement problems, maintenance optimization problems, and bridge inspection problems.

Finally, a dynamic programming model is developed that finds an inspection schedule for a group of suppliers in a infinite planning horizon. In addition, an efficient approximate dynamic programming algorithm is developed to solve the model. 


\title{
CHAPTER 2. ROBUST SUPPLY CHAIN DESIGN CONSIDERING CORRELATED FAILURES AND INSPECTION
}

\begin{abstract}
This chapter studies the impact of correlated supplier failures as well as inspection to detect these failures in the context of a supplier selection problem. Also, this chapter explains explains how this problem can be modeled as a variant of a facility location problem, and develops a two-stage stochastic programming model to explore the tradeoffs between costs and risk when designing a supply chain. The first-stage decisions represent strategic decisions such as location and capacity of suppliers while in the second stage, operational decisions related to transportation and inspection are determined. In the computational results, the effect of supplier correlation and inspection on supplier selection, transportation, and inspection strategies are examined. A sensitivity analysis is also performed to explain the effect of key parameters (capacity, correlation, and inspection) on expected total cost and expected cost of shipped tainted materials.
\end{abstract}

\subsection{Introduction and motivation}

Issues in supply chain are of both practical and theoretical importance, as demonstrated both by the amount of research in this area and the increasing prevalence of supply chain issues in the popular media $[6,7,8]$. The supply chain comes under particular scrutiny when there are failures that either prevent necessary items from being supplied, or cause damaged goods to reach consumers. This dissertation studies how supply chains should be designed to mitigate the effects of supplier failures, and explores how integrating inspection policy decisions into facility location and supply chain design can impact the solutions that are obtained. 
This dissertation is motivated by several recent examples of supply chain failures that occurred in the pharmaceutical and food supply chains. In 2008, tainted heparin - a widely-used anticoagulant produced from the mucous membranes of pig intestines - was widely distributed and administered to patients. The contaminated heparin was responsible for 81 patient deaths and hundreds of allergic reactions in the United States alone. Tainted heparin also affected patients in an additional eleven countries [4]. In 2011, lettuce, cucumbers, and tomatoes were recalled in Germany before ultimately determining that E. coli-contaminated sprouts were responsible for the deaths of 31 people and making more than 3,000 gravely ill [9]. Similarly in the United States in 2010, more than 500 million eggs were recalled after salmonella-tainted eggs made more than 1,500 people ill [10]. These examples illustrate how serious supply chain failures can be, particularly when compromised goods reach consumers.

Motivated by these cases, this dissertation considers supplier sourcing in twotiered supply chains. First-tier suppliers are those who produce a product from raw materials or subassemblies. In the case of heparin, for example, this dissertation assumes that pharmaceutical manufacturers such as Baxter are the first-tier suppliers. Second-tier suppliers harvest or produce the raw materials or subassemblies that are ultimately shipped to the first-tier suppliers. In the heparin example, pig farmers who sell their pigs to pharmaceutical companies are examples of second-tier suppliers. This dissertation assumes that customers (typically healthcare or group purchase organizations) must partner with one or more first-tier suppliers to obtain the required demand for quality products. Therefore, "opening a facility" in these models is equivalent of entering a long-term contractual agreement with a first-tier supplier. Although the suppliers typically produce high-quality product, they can fail and produce (at least partially) tainted product. Such failures require customers to build in redundant capacity that can be used to obtain quality product in the face of supplier failures. 
One of the issues raised by the motivating examples is the issue of correlated supplier failure. In the case of heparin, quality problems were ultimately traced to the contaminated pig intestines used to produce the drug. Blue pig ear disease, a highly-contagious reproductive and respiratory disease that affects droves of pigs, swept through the region in China where pigs were raised to produce heparin. As a result of consuming tainted raw material, at least 12 Chinese companies produced and shipped tainted heparin throughout the world [11].

These failures were not independent - rather, they were all the result of a disease outbreak that affected an entire region. Similar correlated failures were responsible for the food contamination. E. coli contamination in produce, such as sprouts, is caused when animal waste is used either as a fertilizer or contaminates groundwater. When groundwater becomes contaminated, it can affect many farms in an area. Both of these examples illustrate how supply chain disruptions are often not independent, but instead the result of a shared underlying cause for the disruption. Such correlation in supply failures can also occur for other reasons such as inclement weather, political unrest in a region, or labor issues such as strikes that affect multiple facilities. As a result, it is critical to consider the correlation between suppliers when considering their failure probabilities since supplier failures are often not independent as is typically assumed in this type of research [12].

Another issue raised by these supply chain disruptions is that of supplier inspection. Prior to the deaths and illnesses of patients, heparin plants were rarely inspected by regulatory agencies such as the FDA. However in the aftermath of the heparin incident, an inspection of the Changzhou SPL facility that produced heparin did not have "adequate systems for evaluating the suppliers of crude heparin materials, or the crude materials themselves, to ensure that these materials are acceptable for use" [13].

Subsequent testing was developed to detect tainted heparin. Similarly, food can 
be tested to ensure that it is free of contaminants such as salmonella or E. coli. Had these inspections and tests been performed prior to the shipment of tainted produce, perhaps lives could have been saved and illnesses prevented.

As this example clearly shows, inspection can be an effective method to ensure that only high-quality products reach to customers when a disruption occurs. There is a vast literature on inspection in the context of production systems and supply chains. David et al. [14] study inspection decisions in a production-inventory system with multiple unreliable supply sources. They assume that the units supplied by each supply source have a certain defect rate. They consider two related, sequential decisions. The first set of decisions is the amount of material they should order from each supplier to satisfy the customers' demand. The second set of decisions is whether to inspect some part of units received from each source and consequently repair any identified defective items. Hariga and Azaiez [15] consider inspection scheduling for a continuous production process which is subject to random failure. The failures are not visible and only revealed by inspection. At any inspection, if failure is detected then repair action is applied to restore the process. A non-Markovian failure process with increasing failure rate is used to formulate the problem and three cost-effective heuristic procedures are developed. Chun [16] designs a Bayesian inspection procedure for a production process, which is subject to a random failure. The model simultaneously determines how often to inspect items on the production line, develops a procedure to identify how to search economically to detect more defective items, and finally decides when to stop the search process and salvage the remaining items. A stochastic dynamic programming approach is used to formulate the renewal-reward process as a profit-maximization model considering several cost factors.

In some supply chains, inventory shortages and unavailability of products are as problematic as the shipment of tainted products. For example, the unavailability of heparin can cause serious complications for surgery patients since substitutions for 
this drug cannot be easily made. Therefore, care should be when selecting suppliers so that products are available even in the face of failures and disruptions. There are several papers that study the effect of product unavailability. For example, Berger et al. [17] developed a decision tree approach to investigate the risk of unavailability of suppliers. Ruiz-Torres and Mahmoodi [18] extend this model by considering various levels of supplier failure probability. Sawik [19] developed single and bi-objective mixed integer programming models for the supplier selection and order allocation problem considering disruption risks. He assumes that supplies are subject to random local disruptions as a result of local labor strike or equipment breakdowns. He also considers global disasters that render supplies from all suppliers disrupted. In his model, disruption risk is controlled through the use of (conditional) value-at-risk approach.

This dissertation falls into the broad category of supply chain design that considers risk. Several typologies have been proposed to categorize risks in the supply chain $[20,21]$. The research problem of this dissertation is best categorized as one that designs supply chains that are robust against supply side disruptions - that is, this research wants locate facilities so that systems are able to perform their intended functions well when the supply chain is transformed due to the failure of suppliers. This research relaxes the assumption that suppliers fail independently of each other, and simultaneously consider whether or not inspection should be instituted at each facility when determining which facilities should be opened. This research shows how this problem can be modeled as a facility location problem under uncertainty. As explained before, opening a facility is entering a long-term contractual agreement with a first-tier supplier. The fixed cost of opening a facility therefore is not the cost of building the facilities, but the legal fees and other costs associated with signing a long-term contract.

For a research overview of facility location under uncertainty, the reader is referred 
to a survey by Snyder [12]. The initial research in this area focused on demand uncertainty - specifically, facility congestion that results from uncertain demand $[e . g$. , $22,23,24,25,26,27]$. Most of this research was motivated by the placement of emergency facilities. More recently, researchers have focused on uncertainty that results from the availability of suppliers. For example, Snyder and Daskin [28] propose an extension of the uncapacitated p-median problem to ensure the robustness of the solutions obtained in the face of supplier failures. In particular, their work that considers not only the operating cost when all facilities are functioning normally, but also the operating cost when facilities fail. In their proposed framework customers are assigned to both a primary facility and to backup facilities in case closer facilities have failed. They propose a Lagrangian relaxation algorithm to solve this problem, and explore the tradeoff between the cost to construct the network and the operating cost when the network fails.

Motivated by areas that are particularly prone to disruption (e.g., Gulf Coast states during hurricane season), Cui et al. [29] extend this work to consider sitedependent failure probabilities. They propose a Lagrangian Relaxation algorithm to solve this problem, and a continuous approximation algorithm to predict the total system cost without explicitly obtaining solutions, which is particularly valuable for large-scale instances of this problem. Gade and Pohl [30] introduce a capacitated facility location problem considering supplier failure. They model this problem as a two-stage stochastic program, and solve the problem using sample average approximation. Additionally, Peng et al. [31] propose a heuristic to solve the p-robustness problem, which ensures that the operating cost under disruption is within an acceptable factor of the normal operating costs. Their work generalizes the uncapacitated facility design problem in that it considers multiple echelons of the supply chain (e.g., determining transshipment nodes in addition to supply nodes).

Additional related research has been completed by Lee [32] who develops heuristics 
to solve the p-median problem with supplier unreliability; Bundschuh et al. [33] who considers an n-tiered supply chain with sourcing limits; Gaonkar and Viswanadham [20] who develop two quadratic integer linear programs for supplier selection problems that minimize the total expected cost or that minimize the expected shortfall; and Iakovou et al. [21] which considers a variation of the news vendor problem that maximizes total profit and captures the tradeoff between optimal inventory policy and supply chain protection level.

Although these are the most closely-related research papers to our own, there is a large and diverse literature on problems that require joint strategic and operational decisions, mostly considering multiple objectives, that are relevant to our problem. Lin and Kwok [34], Caballero et al. [35], Yildiz et al. [36] present metaheuristics for multi-objective location routing problems. Yildiz et al. [37] present joint transportation decision making among multiple customers and suppliers along with a strategic crossdock location decision. The model of this research can be modified to incorporate several practical considerations included in these papers.

This research considers an extension of the capacitated facility location problem. Also, it considers correlated supplier failures and integrates the operational decision of whether or not to inspect suppliers while making long-term decision of supplier should be selected. This adds to the growing body of literature that integrates tactical level decisions when making strategic level facility location decisions (e.g., Erlebacher and Meller [38], Daskin et al. [39] which consider the integration of facility location and inventory decisions; Min et al. [40], Nagy and Salhi [41] which offer surveys of location-routing problems).

The previous research on correlated supplier failures is limited. For example, Li and Ouyang [42] studied spatial correlation among facility disruptions in the context of the reliable uncapacitated fixed charge location problem. They developed a model to minimize the costs under normal and failure scenarios by considering different 
structure of the spatial correlation. They use the continuum approximation method to solve their model. Liberatore et al. [43] present a tri-level formulation to optimize the protection plan for a capacitated median problem considering disruptions. The objective is to reduce the impact of the worst-case loss of non-fortified facilities, by fortifying facilities in the present system where the disruption can occur in large areas. They analyze the impact of partial or complete disruptions based on the correlation effects between the facilities. They proposed an exact solution approach based on a tree-search procedure.

This dissertation makes a number of contributions to the literature. First, it relaxes the assumption traditionally made that the failure of suppliers is independent. In practice, this is often not the case as illustrated by the heparin and food examples shared above. It is explained how correlated failures can be incorporated into the model via the generation of scenarios that are used in the stochastic program. The computational results show that this has a significant impact both on the cost of the obtained solutions, and the solutions themselves. Second, this dissertation integrates the strategic decisions of where facilities should be opened with operational level decisions of whether or not inspection should be instituted at each facility. Third, this research relaxes the assumption that supplier failures are absolute - that is, partial failures of suppliers are allowed in the proposed model (i.e., some of the supplies produced from a facility are damaged whereas others are not). This is an important consideration when the underlying cause of failures is due to the second-tier suppliers as demonstrated by the motivating examples shared above. In this case, the quality problems may or may not appear in all of the first-tier suppliers depending on their quality practices and the second-tier suppliers they work with. To the best of our knowledge, partial failures have not been considered in the literature before. Finally, a comprehensive two-stage stochastic program is developed, which adds to the growing body of research on robust facility location by considering correlated scenarios and 
incorporating inspection decisions in the second stage. This model is exercised to provide insights about how key parameters (capacity, correlation, inspection) impact on the obtained solutions and the expected total cost of these solutions.

The remainder of the chapter is outlined as follows. Section 2.2 explains the problem and detail of mathematical model and the solution methodology is used to solve this problem. Section 2.3 exercises this model and analyzes the effect of capacity, supplier quality, correlation, and inspection both on the selected suppliers, and the total cost of the obtained solutions. Finally Section 2.4 offers conclusions.

\section{$2.2 \quad$ Model}

A two-stage stochastic program is developed to model the Robust Supply Chain Design with Correlated Failures and Inspection (RSCDCFI). As described in the introduction, this research considers supplier sourcing in two-tiered supply chains. Recall that first-tier suppliers produce a product from raw materials or subassemblies, whereas second-tier suppliers are those who supply the raw materials to the first-tier suppliers. This research assumes that a customer (e.g., group purchase organizations in a pharmaceutical supply chain) have multiple sites and must determine which first-tier suppliers to partner with to fulfill demand at its sites. In this framework, the customer determines which first-tier suppliers should be selected to fulfill the demand across all customer sites during the first stage. After these relationships are established, it is assumed that failures may occur at each of the selected suppliers. When the failures occur, the customer must determine at the start of the second stage how to assign customer sites to suppliers.

This research assumes that the failure of first-tier suppliers occurs when secondtier material suppliers supply them with contaminated raw materials as occurred in the heparin outbreak. If this occurs, then it is assumed that all first-tier suppliers receiving tainted raw materials will produce damaged product. Because in this research 
failures arise as a function of tainted raw materials, it is assumed that the degree of failure is proportional to the amount of tainted raw material that they receive instead of binary - that is, a facility can be open and producing some materials that are good and some which are tainted. The framework is used to generate and describe these failures is described in detail in Section 2.2.3.

The scenario generation requires three types of information: a marginal mean vector, a correlation matrix, and cumulative density function (cdf) for tainted production. The marginal mean is a measure of quality at the facility, and represents the long term percentage that some of its production is tainted. The correlation between two facility failures is proportional to the number of common second-tier suppliers they use. The cumulative density function for tainted production determines the extent of a random disruption. The data for these parameters can be obtained from historical data. In the case of pharmaceutical supply chains, for example, the Food and Drug Administration (FDA) requires inspection of pharmaceutical suppliers every two years to ensure that only high-quality drugs reach to patients [44]. An inspection can reveal that a supplier is in an out-of-compliance state for a number of reasons including operator errors, machine errors, or defective raw materials. The outcome of these inspections can be used to estimate the marginal mean. Moreover, the cdf for tainted production can be calculated from defect rates calculate by quality assurance procedures.

This research assumes that in addition to determining which first-tier suppliers to build relationships with, customers can also decide whether or not to institute inspection at each facility. If inspection is instituted, it is assumed that this inspection will detect and discard a proportion of the tainted material that is produced at the facility. If the customer forgoes the opportunity to inspect the materials at a facility, it is assumed that all of the tainted materials produced at that facility reach the customer. If inspection is not instituted, customers will likely be assigned to facilities 
that are producing damaged goods since tainted materials cannot be detected. If, however, inspection is instituted then the model will determine which customers to assign to which site and may determine that some demand should go unsatisfied.

The objective of proposed model is to minimize total expected cost. In the model, five types of costs are considered:

1. Fixed costs - these costs are one-time costs that represent the costs required to establish a formal relationship with the first-tier suppliers (e.g., initial inspection and legal costs to establish a contractual relationship). These costs are analogous to those required to open a facility in a facility location framework. It is assumed that the fixed costs are proportional to the capacity of the facilities (i.e., higher capacity leads to a higher fixed cost).

2. Variable costs - these costs represent the cost of producing and transporting material to fulfill customer orders. Note that it is assumed that these variable costs must be paid whether the material produced is quality product or tainted.

3. Shortage costs - these costs represent the costs that are incurred for each unit of customer demand is not satisfied (e.g., lost sales). Shortage costs are very high, as the unavailability of product can cause serious supply chain issues (e.g., the unavailability of heparin may result in significant health complications).

4. Cost of tainted material - these costs are incurred when damaged material reaches the customer. Tainted material costs are very high, as they cause significant damage to consumers or the reputation of the company (e.g., damaged heparin reaching a patient).

5. Inspection cost - this represents the cost of instituting inspection at a supplier facility. If the decision to inspect products is made, it must be paid whether or not tainted material is detected at supplier facilities. However, if tainted mate- 
rials are found, most of the materials detected and discarded prior to reaching the customer.

Since the objective of the problem is to minimize the total expected cost, the mathematical model must effectively trade off the costs of installing capacity, shorting customers, shipping tainted materials, and inspecting material against each other.

In the following subsections, notation is defined first, which is followed by the two-stage stochastic formulation of the Robust Supply Chain Design with Correlated Failures and Inspection.

\subsubsection{Sets and Parameters}

- $I$ is the set of customers $(i \in I)$.

- $L$ is the set of candidate facility locations $(l \in L)$. Included in the set $L$ is a dummy facility indexed by $m$ (with no fixed cost: $f_{m}=0$ ) which can satisfy all demand.

- $f_{l}$ is the fixed cost of opening a facility at candidate location $l$.

- $c_{l}$ is the capacity at candidate location $l$.

- $n_{l}$ is the fixed cost of inspection at candidate location $l$.

- $b_{i}$ is the total demand of customer $i$.

- $d_{i l}$ is the per unit cost of satisfying demand from customer $i$ from location $l$.

- $u_{i}$ is the per unit cost of customer $i$ 's unsatisfied demand (i.e., $d_{i m}=u_{i}$ ).

- $t_{i}$ is the cost per unit of "tainted" material shipped to customer $i$.

- $S$ is the set of scenarios $(s \in S)$. A scenario is defined as an event where facilities that belong to a subset $J \subseteq L$ of candidate facility locations that have failed. It is assumed that plausible $S$ are known in advance. 
$-s^{\prime} \in S$ represents the scenario where all facilities are working normally.

$-p_{s}$ is the probability of scenario $s$ occurring.

- $q_{l s}$ represents the fraction of tainted material produced at facility $l$ under scenario $s$ (one can also consider this as the probability that an item produced is tainted). Note that $q_{m s}=0$, i.e., the dummy facility $m$ never fails.

- $r_{l s}$ represents the fraction of tainted material produced at facility $l$ under scenario $s$ after inspection, i.e., the proportion of tainted material that is not detected even after inspection and still reaches the customer. Note that if inspection is used $100\left(q_{l s}-r_{l s}\right) \%$ of material produced is discarded.

- To make the definitions of $q_{l s}$ and $r_{l s}$ clearer, consider the following example. Suppose that under scenario $s$, the extent of failures at unreliable facility $l$ is given by $q_{l s}=0.2$ and $r_{l s}=0.05$. This means that for every 100 units of material produced at facility $l, 20$ of them will be tainted. In the event of no inspection, all of these 20 tainted units will be shipped to customers. If inspected, 15 of these 20 tainted units will be detected and discarded while 5 of them will go undetected and be shipped to customers.

\subsubsection{Stochastic Programming Formulation}

The following decision variables are defined as part of the formulation:

- $x_{l}= \begin{cases}1, & \text { if the facility } l \text { is opened } \\ 0, & \text { otherwise }\end{cases}$

- $y_{i l} \in[0,1]$ is the proportion of production capacity at facility $l$ assigned to customer $i$.

- $w_{i l} \in[0,1]$ is the proportion of demand of customer $i$ satisfied by facility $l$. 
- $v_{i l} \in[0,1]$ is the tainted proportion of the demand of customer $i$ supplied by facility $l$.

- $z_{l}= \begin{cases}1, & \text { if inspection is used at facility } l \\ 0, & \text { otherwise }\end{cases}$

A two-stage stochastic program for making facility location and facility-to-customer assignments that minimizes overall expected costs can be written as follows:

$$
\begin{aligned}
\min & \sum_{l \in L} f_{l} x_{l}+E[Q(\mathbf{x}, \tilde{s})] \\
\text { subject to } & x_{m}=1 \\
& x_{l} \in\{0,1\}, \quad \forall l \in L
\end{aligned}
$$

where $\sum_{l \in L} f_{l} x_{l}$ is the fixed cost of opening facilities. Also, $E[\cdot]$ is the expectation function taken with respect to random scenario $\tilde{s}$ and $Q(\mathbf{x}, \tilde{s})$ is the optimal solution of the second stage problem: 


$$
\begin{aligned}
\min \quad & \sum_{i \in I} \sum_{l \in L} b_{i} d_{i l} w_{i l}+\sum_{i \in I} b_{i} u_{i} w_{i m}+\sum_{i \in I} \sum_{l \in L} b_{i} t_{i} v_{i l}+\sum_{l \in L} n_{l} z_{l} \\
\text { subject to } \quad & \sum_{i \in I} y_{i l} \leq x_{l}, \quad \forall l \in L \\
& \sum_{l \in L} w_{i l}=1, \quad \forall i \in I \\
& b_{i} w_{i l} \leq c_{l} y_{i l}, \quad \forall i \in I, l \in L \\
& b_{i} w_{i l} \leq\left(1-\left(q_{l \tilde{s}}-r_{l \tilde{s}}\right)\right) c_{l} y_{i l}+c_{l}\left(1-z_{l}\right), \quad \forall i \in I, l \in L \\
& b_{i} v_{i l} \leq q_{l \tilde{s}} c_{l} y_{i l}, \quad \forall i \in I, l \in L \\
& b_{i} v_{i l} \leq r_{l \tilde{s}} c_{l} y_{i l}+q_{l \tilde{s}} c_{l}\left(1-z_{l}\right), \quad \forall i \in I, l \in L \\
& v_{i l} \geq q_{l \tilde{s}} w_{i l}-z_{l}, \quad \forall i \in I, l \in L \\
& v_{i l} \geq r_{l \tilde{s}} w_{i l}-\left(1-z_{l}\right), \quad \forall i \in I, l \in L \\
& y_{i l}, w_{i l}, v_{i l} \in[0,1], \quad \forall i \in I, l \in L \\
& z_{l} \in\{0,1\}, \quad \forall l \in L .
\end{aligned}
$$

Here $x_{l}$ are first and $y_{i l}, w_{i l}, v_{i l}$, and $z_{l}$ are second stage decision variables respectively. Part of the data in the second stage problem depends on random scenario $\tilde{s}$, which has the discrete distribution $\operatorname{Prob}\{\tilde{s}=s\}=p_{s}$. Since the set $L$ includes a dummy facility $m$ (the expected cost of unsatisfied demand is calculated by considering dummy facility) which can satisfy all the demand and never fails, for every $\mathbf{x}$ in the set $\left\{\mathbf{x}: x_{m}=1, x_{l} \in\{0,1\}, l \in L\right\}$, the feasible set of the second stage problem (2.4)-(2.14) is nonempty for all $s \in S$. Therefore, the recourse is relatively complete (i.e., every possible solution $x$ in the first stage has a feasible completion in the second stage) and, for any realization $\tilde{s}=s, Q(\mathbf{x}, s)<\infty$. This implies that the following expectation always exists: $E[Q(\mathbf{x}, \tilde{s})]=\sum_{s \in S} p_{s} Q(\mathbf{x}, s)$.

The Objective (2.1) in the first stage problem is the sum of fixed cost of opening facilities and overall expected costs. The first stage Constraint (2.2) ensures that the 
dummy facility with infinite capacity is always open. The other first stage constraint (2.3) restricts the location variables, $x_{l}$, to be binary. Given a feasible first stage solution vector $\mathbf{x}$, the Objective (2.4) of the second stage problem for random scenario $\tilde{s}$ minimizes the sum of the transportation cost $\left(d_{i l}\right)$, the cost of unsatisfied demand $\left(u_{i}\right)$, the cost of supplying tainted material $\left(t_{i}\right)$, and the cost of inspection $\left(z_{l}\right)$. Constraint (2.5) requires a facility to be open if any portion of customer demand is served from the facility. In addition, it ensures that the total customer demand $\left(b_{i}\right)$ assigned to any facility $(l)$ cannot exceed the facility's capacity. Constraint (2.6) requires that the entire demand of every customer $(i)$ is met, possibly with some portion satisfied by the dummy facility $m$. Constraints (2.7) and (2.8) calculate the amount of product that is shipped to customer $i$ from facility $l$ to satisfy a customer's demand. In particular, if inspection is not implemented, $z_{l}=0$ and Constraint (2.8) becomes inactive. In this case, Constraint (2.7) becomes binding and ensures that the amount shipped is the proportion of facility l's capacity dedicated to customer $i$. However, if inspection is implemented, $z_{l}=1$ and Constraint (2.8) becomes binding. This will adjust the amount of product shipped to account for the tainted product that is caught in inspection and discarded. Constraints (2.9) and (2.10) together trade the amount of tainted material that is shipped to the customer. Without inspection, Constraint (2.9) implies that all of the tainted goods will reach the customer. With inspection, Constraint (2.10) implies that only material passing inspection will be shipped to the customer. Constraints (2.11) and (2.12) together ensure that the tainted proportion of the demand of customer $i$ supplied by facility $l$ is higher than total amount of tainted material send from facility $l$ to customer $i$, with or without implementing the inspection. Constraints (2.13) and (2.14) indicate the continuous and binary decision variables in the second stage. 


\section{Scenario-based Formulation}

This part presents the deterministic equivalent (i.e., RSCDCFI) of the formulation (2.1)-(2.14). Note that the second stage decision variables are now indexed by a scenario index, s. The objective and constraints are similar to those presented in Section 2.2.2.

$$
\begin{array}{ll}
\min & \sum_{l \in L} f_{l} x_{l}+\sum_{s \in S} p_{s}\left(\sum_{i \in I} \sum_{l \in L} b_{i} d_{i l} w_{i l s}+\sum_{i \in I} b_{i} u_{i} w_{i m s}+\sum_{i \in I} \sum_{l \in L} b_{i} t_{i} v_{i l s}+\sum_{l \in L} n_{l} z_{l s}\right) \\
& x_{m}=1 \\
& \sum_{i \in I} y_{i l s} \leq x_{l}, \quad \forall l \in L, s \in S \\
& \sum_{l \in L} w_{i l s}=1, \quad \forall i \in I, s \in S \\
& b_{i} w_{i l s} \leq c_{l} y_{i l s}, \quad \forall i \in I, l \in L, s \in S \\
& b_{i} w_{i l s} \leq\left(1-\left(q_{l s}-r_{l s}\right)\right) c_{l} y_{i l s}+c_{l}\left(1-z_{l s}\right), \quad \forall i \in I, l \in L, s \in S \\
& b_{i} v_{i l s} \leq q_{l s} c_{l} y_{i l s}, \quad \forall i \in I, l \in L, s \in S \\
& b_{i} v_{i l s} \leq r_{l s} c_{l} y_{i l s}+q_{l s} c_{l}\left(1-z_{l s}\right), \quad \forall i \in I, l \in L, s \in S \\
& v_{i l s} \geq q_{l s} w_{i l s}-z_{l s}, \quad \forall i \in I, l \in L, s \in S \\
& v_{i l s} \geq r_{l s} w_{i l s}-\left(1-z_{l s}\right), \quad \forall i \in I, l \in L, s \in S \\
& y_{i l s}, w_{i l s}, v_{i l s} \in[0,1], \quad \forall i \in I, l \in L, s \in S \\
& z_{l s} \in\{0,1\}, \quad \forall l \in L, s \in S \\
& x_{l} \in\{0,1\}, \quad \forall l \in L
\end{array}
$$

\subsubsection{Scenario Generation for Correlated Facility Failures}

As explained in the introduction, many real-world problems have correlation between facility failures. Although such correlated failures can have a substantial impact on the operations of a supply chain, they have not been considered previously. One straightforward way of accounting for correlation in robust supply chain design is 
to incorporate it into scenario generation. Although there are several other models which utilize scenario-based approaches that could be adapted to do this [e.g., 19, 31], they have not explored the effect of this important real-world phenomenon previously. As mentioned earlier, failure at a first-tier supplier is not absolute. In other words, after a failure a facility may still be producing materials; some of these will be highquality, whereas others will be tainted. It is assumed that main cause of failures is low-quality raw materials from the second-tier suppliers but the amount of tainted material produced as a result of failure is ultimately determined by a facility's internal quality practices. Therefore, first correlated binary variables to indicate failure must be generated and then the fractions of tainted products (i.e., the extend of a failure) must be simulated given that a failure has occurred. This scenario generation method is comprised of the following two steps, as described in detail below:

1. Simulate correlated binary variables representing whether a facility has failed or not.

2. If a facility has failed, simulate the fraction tainted of production at that facility.

\section{Step 1: Simulation of Correlated Binary Variables:}

In order to simulate correlated failures in Step 1 above, a method described by Qaqish [45] for generating $L$ binary correlated variables $Y_{1}, Y_{2}, \ldots, Y_{L}$ is adapted. Here, $Y_{l}$, $l=1,2, \ldots, L$, is a Bernoulli random variable, which takes the value of 1 if some portion (not necessarily 100\%) of production at facility $l$ is tainted; 0 otherwise. Qaqish's method [45] was selected for its flexibility in terms of not requiring equal marginal means and allowing unpatterned correlation and its computational efficiency. Qaqish's method [45] requires two inputs: a marginal mean vector, and a correlation matrix.

It is assumed that marginal mean of facility $l$, denoted by $\mu_{l}$, represents the longrun percentage that some fraction of its production is tainted. In order words, a high 
marginal mean implies that a facility has low quality standards therefore frequently produces tainted materials. It is also assumed that the marginal means are available through historical data and expert knowledge.

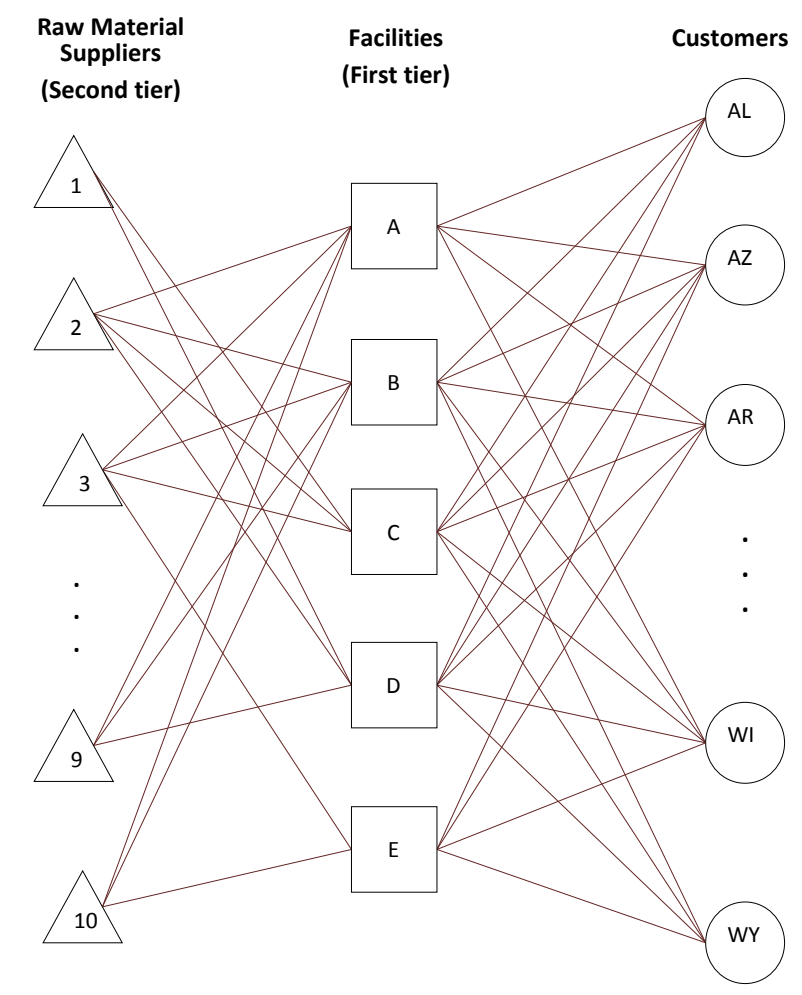

Figure 2.1: Two-tier supply supply chain.

Motivated by the heparin example, a two-tier supply chain is assumed in order to obtain the correlation matrix (see Figure 2.1). The facilities (e.g., heparin manufacturers) represent the first-tier and the raw material suppliers (e.g., pig farms) represent the second-tier. Correlation between two facilities is determined by a simple ratio of number of common raw material suppliers that they share to the total number of raw material suppliers that they use. As an example, consider the two-tier supply chain in Table 2.1 and its corresponding correlation matrix in Table 2.2. If a facility works with a particular raw material supplier, the corresponding entry in Table 2.1 is 1 ; 0 otherwise. For instance, according to this simple approach for cal- 
culating correlation, the correlation between Facility A and Facility B is $6 / 8=0.75$ since there are six common raw material suppliers that both facilities work with and the total number of raw material suppliers used by either of the two facilities is eight. Despite its simplicity, this formula is reasonable for many real-world situations, where facilities prefer working with raw material suppliers that are geographically close and a natural disaster or a pandemic can affect many neighboring raw material suppliers simultaneously.

Table 2.1: Example of a two-tier supply chain.

\begin{tabular}{c|ccccc}
\hline Raw Material Suppliers & Facility A & Facility B & Facility C & Facility D & Facility E \\
\hline 1 & 0 & 0 & 1 & 1 & 0 \\
2 & 1 & 1 & 1 & 1 & 0 \\
3 & 1 & 1 & 1 & 0 & 1 \\
4 & 1 & 0 & 0 & 1 & 0 \\
5 & 0 & 0 & 0 & 1 & 1 \\
6 & 1 & 1 & 1 & 0 & 0 \\
7 & 1 & 1 & 0 & 0 & 0 \\
8 & 1 & 1 & 0 & 0 & 0 \\
9 & 1 & 0 & 1 & 0 & 0 \\
10 & 1 & 1 & 0 & 0 & 1 \\
\hline
\end{tabular}

Table 2.2: Correlation matrix of the two-tier supply chain in Table

\begin{tabular}{c|ccccc}
\hline & $A$ & $B$ & $C$ & $D$ & $E$ \\
\hline$A$ & 1 & 0.750 & 0.444 & 0.200 & 0.222 \\
$B$ & 0.750 & 1 & 0.375 & 0.111 & 0.286 \\
$C$ & 0.444 & 0.375 & 1 & 0.285 & 0.143 \\
$D$ & 0.200 & 0.111 & 0.285 & 1 & 0.167 \\
$E$ & 0.222 & 0.286 & 0.143 & 0.167 & 1 \\
\hline
\end{tabular}

Given the marginal means and correlation matrix, Qaqish [45] describes an efficient procedure to calculate conditional probabilities $\operatorname{Prob}\left\{Y_{l}=1 \mid \mathbf{X}_{l}=\mathbf{x}_{l}\right\}, l=2,3, \ldots, L$, where $\mathbf{X}_{l} \equiv\left[Y_{1}, Y_{2}, \ldots, Y_{l-1}\right]^{\top}$. Using these conditional probabilities, the Bernoulli random variables $Y_{1}, Y_{2}, \ldots, Y_{L}$ representing facility failures can be easily simulated. 


\section{Step 2: Simulation of Tainted Production at Failed Facilities:}

Let $Q_{l}$ be a random variable representing the fraction of tainted material produced at facility $l$. When $Y_{l}=0$ (i.e., facility $l$ is operating under normal conditions), the value of $Q_{l}$ is also zero, which means that facility $l$ produces $100 \%$ high quality material. However, when $Y_{l}=1$ (facility $l$ has failed), facility $l$ produces tainted material, the quantity of which is determined by random variable $T_{l}$ having a known cumulative density function $(\mathrm{CDF}) F_{l}$ with support $[0,1]$. Therefore,

$$
Q_{l}=Y_{l} \times T_{l}
$$

It is assumed that the CDF $F_{l}$ of random variable $T_{l}$ can be obtained from historical data, quality inspections, and expert knowledge.

Here $R_{l}$ denotes the random variable representing the fraction of tainted material produced at facility $l$ after inspection. It is assumed that $R_{l}$ is a function of $Q_{l}$ :

$$
R_{l}=g_{l}\left(Q_{l}\right)
$$

Figure 2.2 gives an outline of the scenario generation procedure for correlated facility failures.

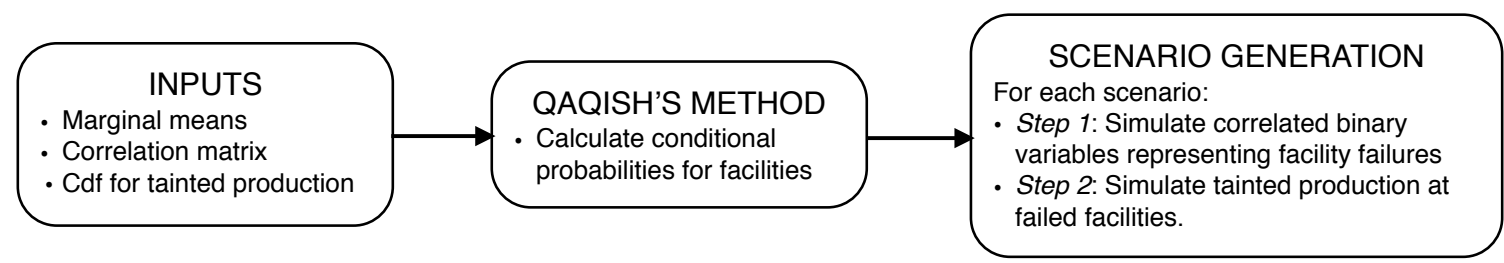

Figure 2.2: Outline of scenario generation procedure for correlated facility failures. 


\subsubsection{Solution Methodology}

RSCDCFI is a two-stage mixed-binary stochastic program. To solve it, the integer L-shaped method for stochastic integer programs developed by Laporte and Louveaux [46] is used. It is a general branch-and-cut procedure similar to the well-known Lshaped method for classical two-stage linear stochastic programs developed by Slyke and Wets [47], but it allows first stage decision variable to be binary and the second stage decision variables to be discrete or continuous. In numerical experiments, it was observed that the running time of the integer L-shaped method increases dramatically as the number of candidate facility locations increases. To improve solution time, the improved optimality cuts described by Laporte and Louveaux [46] are implemented. Even though improved optimality cuts reduced the solution time, it is believed that the recently proposed integer stochastic programming algorithms [e.g., 48, 49, 50, 51] may better be able to solve large-scale instances of RSCDCFI, and should be considered in future research. The objective in this dissertation is to gain insights about how key parameters (e.g., capacity, correlation, inspection) impact the solutions and the expected total cost of these solutions. The ability to solve large-scale instances is left for future study.

\subsection{Computational Experiments}

In this section, several computational experiments are conducted to gain insights into the effect of capacity, correlation, and inspection on supplier selection and total cost. A workstation with two $3.066 \mathrm{GHz}$ Quad-Core Intel Xeon processors and $32 \mathrm{~GB}$ memory is used to run all experiments. Both the scenario generation procedure (see Section 2.2.3) and the integer L-shaped method (see Section 2.2.4) are implemented in Python. Gurobi Optimizer 4.5.1 is used to solve both the master and subproblem in the the integer L-shaped method.

In the experimental design, six US cities listed in Table 2.3 consider as candidate 
facility locations and the capitals of all continental US states as customers. Although it could be argued that it would have been preferable to apply the model (i.e., RSCDCFI) to a large-scale realistic supply chain design problem, the main goal in this dissertation is to clearly show the effect of supplier correlation and inspection on supplier selection, transportation, and inspection strategies. As a result, a small but nontrivial example is used. A relatively large number of scenarios are also considered to capture the effect of correlated random supplier failures. Specifically, 500 scenarios are considered while large-scale examples of two-stage stochastic mixed-binary programs reported recently use only up to 100 scenarios [50, 51, 52]. The deterministic equivalent of the proposed two-stage stochastic mixed-binary with random recourse model includes 432,000 continuous variables, 3,006 binary variables and 1,326,007 constraints.

150 points were randomly generated in the US as locations of raw material suppliers. If a raw material supplier falls within a pre-specified distance from a particular facility, it is assumed that raw material supplier is assigned to that facility (see Figure 2.3); further details are discussed below.

\begin{tabular}{cc}
\hline Facility & Location \\
\hline 1 & Seattle \\
2 & Los Angeles \\
3 & Denver \\
4 & Chicago \\
5 & Atlanta \\
6 & Boston \\
\hline
\end{tabular}

Table 2.3: Candidate facility locations. 


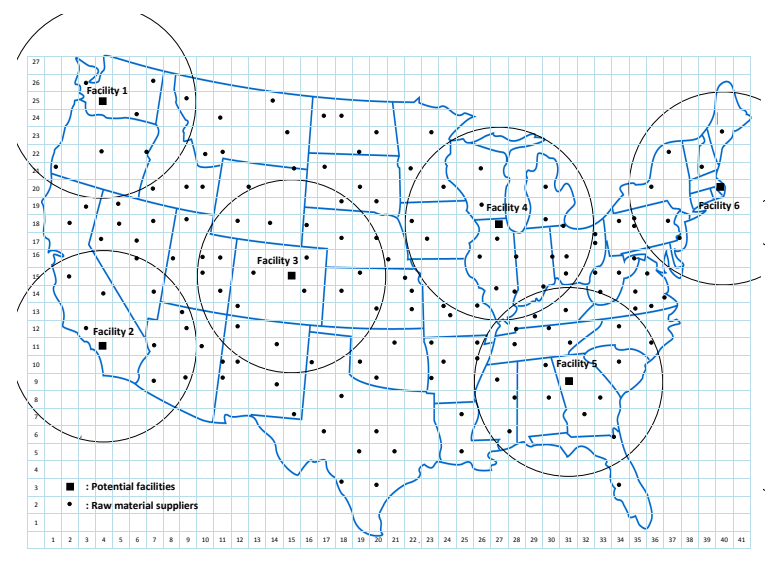

(a) radius $=5$

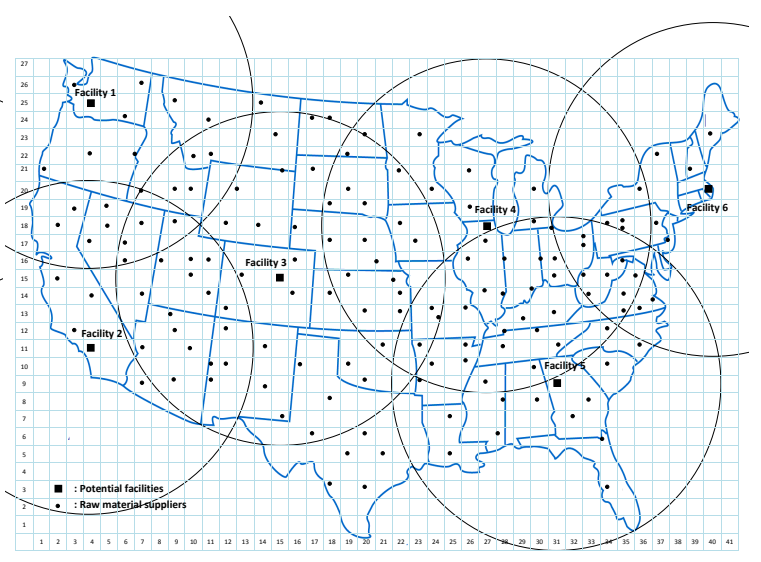

(b) $\quad$ radius $=9$

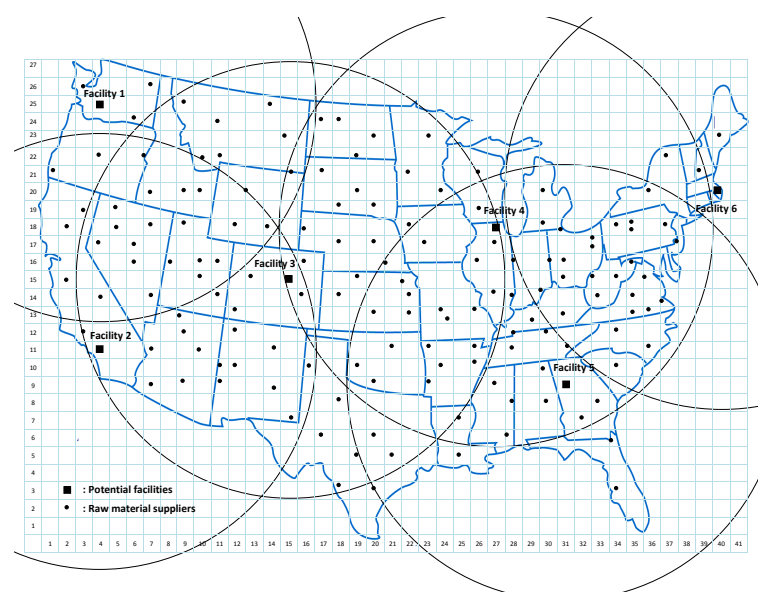

(c) radius $=12$

Figure 2.3: Candidate facility locations, raw material supplier locations, and customers.

In computational experiments, the following factors are varied to study the effect of capacity, supplier quality, correlation, and inspection on supplier selection and total cost:

a) Radius of circular regions centered at candidate facility locations.

The circular regions shown in Figure 2.3 are used to determine the raw material suppliers of a facility. As the radius of the circles increase, facilities will share larger numbers of common raw material suppliers. This implies that larger radii result in higher correlation (recall the simple correlation formula defined 
in Section 2.2.3). Three different radii are used as shown in Figure 2.3. The resulting correlation matrices are shown in Tables 2.4, 2.5, and 2.6.

Table 2.4: Correlation matrix (radius $=5$ ).

\begin{tabular}{c|cccccc}
\hline $\mathrm{R}=5$ & facility 1 & facility 2 & facility 3 & facility 4 & facility 5 & facility 6 \\
\hline facility 1 & 1 & 0 & 0 & 0 & 0 & 0 \\
facility 2 & 0 & 1 & 0 & 0 & 0 & 0 \\
facility 3 & 0 & 0 & 1 & 0 & 0 & 0 \\
facility 4 & 0 & 0 & 0 & 1 & 0 & 0 \\
facility 5 & 0 & 0 & 0 & 0 & 1 & 0 \\
facility 6 & 0 & 0 & 0 & 0 & 0 & 1 \\
\hline
\end{tabular}

Table 2.5: Correlation matrix (radius $=9$ ).

\begin{tabular}{c|cccccc}
\hline $\mathrm{R}=9$ & facility 1 & facility 2 & facility & facility 4 & facility 5 & facility 6 \\
\hline facility 1 & 1 & 0.19 & 0.08 & 0 & 0 & 0 \\
facility 2 & 0.19 & 1 & 0.27 & 0 & 0 & 0 \\
facility 3 & 0.08 & 0.27 & 1 & 0.13 & 0.01 & 0 \\
facility 4 & 0 & 0 & 0.13 & 1 & 0.44 & 0.14 \\
facility 5 & 0 & 0 & 0.01 & 0.44 & 1 & 0.17 \\
facility 6 & 0 & 0 & 0 & 0.14 & 0.17 & 1 \\
\hline
\end{tabular}

Table 2.6: Correlation matrix (radius $=12$ ).

\begin{tabular}{c|cccccc}
\hline $\mathrm{R}=12$ & facility 1 & facility 2 & facility & facility 4 & facility 5 & facility 6 \\
\hline facility 1 & 1 & 0.45 & 0.28 & 0 & 0 & 0 \\
facility 2 & 0.45 & 1 & 0.37 & 0 & 0 & 0 \\
facility 3 & 0.28 & 0.37 & 1 & 0.28 & 0.13 & 0 \\
facility 4 & 0 & 0 & 0.28 & 1 & 0.66 & 0.34 \\
facility 5 & 0 & 0 & 0.13 & 0.66 & 1 & 0.39 \\
facility 6 & 0 & 0 & 0 & 0.34 & 0.39 & 1 \\
\hline
\end{tabular}

b) Marginal means of failure.

As mentioned above, the marginal mean of a facility represents the long-run percentage that some fraction of its production will be tainted. Thus, a high 
marginal mean implies that a facility has low quality standards, and vice versa. Two cases for marginal means are considered as shown in Table 2.7. In Case 1 , the means of failure for facilities over the range $[0.015,0.06]$ were arbitrarily selected. In Case 2, the marginal means for all facilities are set to 0.02 .

Table 2.7: Cases for marginal means of facility failures.

\begin{tabular}{c|cccccc}
\hline & $\mu_{1}$ & $\mu_{2}$ & $\mu_{3}$ & $\mu_{4}$ & $\mu_{5}$ & $\mu_{6}$ \\
\hline Case 1 & 0.025 & 0.02 & 0.015 & 0.06 & 0.05 & 0.015 \\
Case 2 & 0.02 & 0.02 & 0.02 & 0.02 & 0.02 & 0.02 \\
\hline
\end{tabular}

c) Capacity of candidate facilities.

48 random demands were generated over the range of [100,300] and assigned these demands to customers based on their population. The same demands were used for all experiments.

Four cases for facility capacities were considered as shown in Table 2.8. In all four cases, the capacity of all candidate facilities $\left(c_{l}\right)$ are equal for all locations l. In Case 1, the total capacity is equal to the total demand. In Cases 2, 3 and 4 , the total capacity is $5 \%, 30 \%$, and $80 \%$ higher than the total demand, respectively.

Table 2.8: Facility Capacities.

\begin{tabular}{lcc}
\hline & Description & Capacity $\left(c_{l}\right)$ \\
\hline Case 1 & capacity $=$ demand & 1,608 \\
Case 2 & capacity $=(1.05)$ demand & 1,688 \\
Case 3 & capacity $=(1.30)$ demand & 2,090 \\
Case 4 & capacity $=(1.80)$ demand & 2,894 \\
\hline
\end{tabular}

d) Availability of inspection option.

To study the benefits of the inspection option, two cases are considered. In the first case, inspection is not an option. That is, all second stage variables $z_{l s}$ (see 
Section 2.2.2) are set to zero so that inspection is not implemented anywhere in the supply chain. In the second case, the regular stochastic programming formulation (2.15)-(2.27) is used when optimizing the inspection decisions.

Aside from the factors listed above, it is assumed the values of other parameters in the experiments were fixed. These fixed values are given below.

- Fixed cost of opening a facility. Clearly fixed cost of opening a facility depends both on its marginal mean of failure (i.e., quality level) and capacity. Since the facility capacities are set to be equal to each other in all experiments, the fixed cost of a facility by the following simple function of marginal mean:

$$
f=1,150,000-7,500,000 \mu
$$

The fixed cost is further adjusted according to the capacity. For example, if the capacity of a facility is increased by $30 \%$, its fixed cost is also increased $30 \%$.

- Cost of inspection. Inspection cost is assumed to be $\$ 100,000$ for each facility if inspection is instituted.

- Transportation cost. There are $48 \times 6$ arcs in the supply chain from each facility to each customer. $48 \times 6$ random transportations costs were generated over the range $[\$ 100, \$ 1,000]$ and assigned to arcs based on the geographical distances.

- Cost of unsatisfied demand. The per unit cost of unsatisfied demand is $\$ 1,000,000$ for all customers.

- Cost of shipping tainted materials. The cost per unit of tainted material shipped to customers is sampled from a uniform distribution over the range $[\$ 10,000$, $\$ 20,000]$. 
- Tainted Production at Failed Facilities. In the numerical experiments (see Section 2.3), $T_{l}$ for all facilities, $l=1,2, \ldots, L$, is assumed to be a discrete random variable with the following probability mass function:

$$
T_{l}= \begin{cases}0.2, & \text { with probability } 0.2 \\ 0.3, & \text { with probability } 0.5 \\ 0.4, & \text { with probability } 0.3\end{cases}
$$

Furthermore, it is assumed here that there is a simple linear relationship between $R_{l}$ and $Q_{l}$ :

$$
R_{l}=0.05 \times Q_{l},
$$

which means that inspection is able to detect $95 \%$ of tainted materials while $5 \%$ of tainted materials go undetected.

- Number of scenarios. In all experiments, 500 scenarios are used.

\subsubsection{Effect of Capacity}

As described above, fixed cost of opening a facility is proportional to its capacity. Figure 2.4 shows the effect of capacity on expected total cost and expected cost of shipped tainted materials. As expected, it was observed that higher capacity levels for each facility result in fewer opened facilities. As can be seen from Figure 2.4(b), the less redundant capacity there is with respect to total demand, the higher the expected cost of tainted material is. In the extreme case when available capacity equals demand, tainted material is shipped to satisfy demand since the cost of unsatisfied demand is higher than that of shipping tainted material. Even a small increase in capacity (e.g., $5 \%$ more than total demand) results in a substantial reduction in the expected cost of shipped tainted materials. The same trend can be observed for expected total cost in Figure 2.4(a) when capacity is 5\% and 30\% higher than the total demand. 


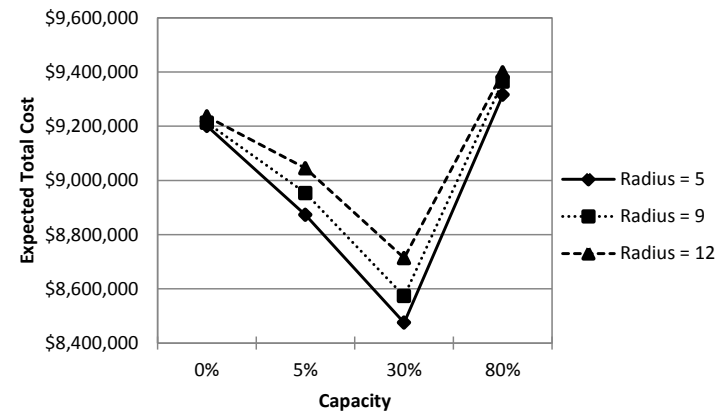

(a) Expected total cost.

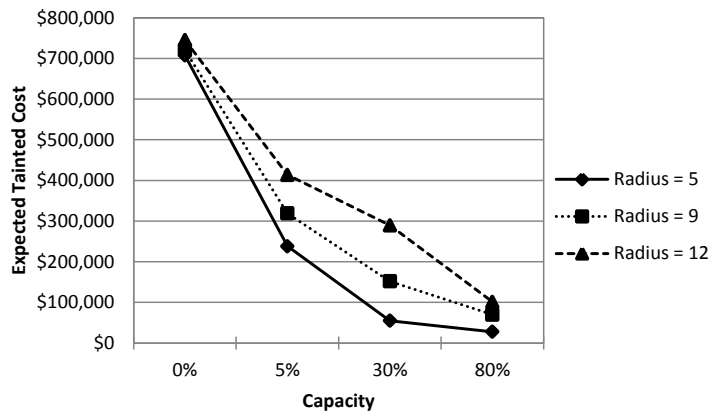

(b) Expected Tainted Cost

Figure 2.4: Effect of capacity on (a) expected total cost and (b) expected cost of shipped tainted materials. Radius determines the level of correlation: radius $=5$ represents no correlation, radius $=9$ medium correlation, and radius $=12$ high correlation. In the experiments, the marginal mean of failure is 0.02 for all facilities.

However, when capacity increased $80 \%$ with respect to total demand, it results in the highest expected total cost in all four cases. This is because it is cheaper to have a modest amount of excess capacity but implement inspection than it is to have a substantial amount of excess capacity. Beyond a certain point, the fixed cost paid for extra capacity outweighs the savings from the tainted material cost. In conclusion, investing in a small amount of redundant capacity while instituting inspection may reduce the cost of shipping tainted materials substantially in the face of facility failures.

\subsubsection{Effect of Correlation}

In order to understand the effect of correlation in facility failures on the design of a supply chain, several failure scenarios with different levels of correlation were generated using the scenario generation method described in Section 2.2.3. Recall that smaller radii imply that the generated failures have little or no correlation between suppliers, whereas larger radii cause facilities to share raw material suppliers and, hence, have correlated failures.

Figures 2.5(a) and 2.5(b) show that higher correlation results in both higher ex- 


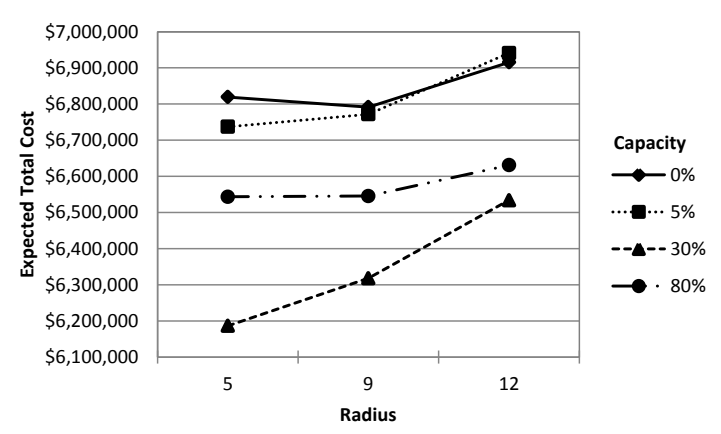

(a) Expected total cost.

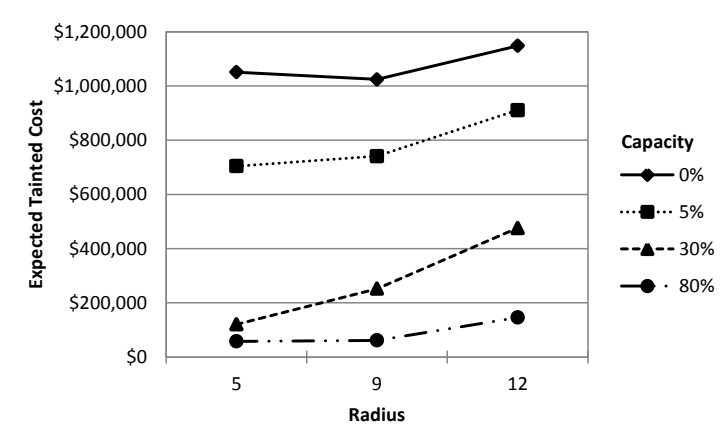

(b) Expected Tainted Cost

Figure 2.5: Effect of correlation on (a) expected total cost and (b) expected cost of shipped tainted materials. Radius determines the level of correlation: radius = 5 represents no correlation, radius $=9$ medium correlation, and radius $=12$ high correlation. In the experiments, the marginal mean vector of facility failures is set to [0.025 0.020 .0150 .060 .050 .015$]$.

pected total cost and expected cost of shipped tainted materials. One interesting observation is that even though the same marginal mean vector is used in these experiments, highly correlated facility failures always lead to higher cost solutions than independent failures (e.g., in Figure 2.3 when radius is 5 , the facility failures are essentially independent; compare to the case when radius is 12 where correlation is very high). In addition, high correlation may lead to different facilities being opened. For example, in the experiments with capacity $30 \%$ more than the demand, while facility 2 was closed in the optimal solutions when radius is 5 and 9 , it was open when radius is 12. A simple explanation for this observation is that more facilities are opened to hedge against the risk of shortage/supply failure when the correlation is high. Hence, it is crucial to take correlations into accounts when designing a supply chain since they may lead to substantially different decisions and higher costs, especially when the correlation is high.

This has important design implications. Models traditionally assume that the failures of facilities are independent. In practice, this is not always true for the reasons described in Section 2.1. However, ignoring this correlation can both underestimate the cost of the solutions obtained and alter the characteristics of the solutions them- 
selves. For this reason, it is critical to account for the correlation of facility failures when determining which facilities to open.

\subsubsection{Effect of Inspection}

Previous work on facility location under uncertainty has not considered widely the operational level decision of whether or not inspection should be instituted at a facility in the face of failure [12]. In order to understand benefits of inspection, two cases are compared. In the first case, inspection is not available. That is; all second stage variables $z_{l s}$ (see Section 2.2.2) are set to zero so that inspection is not implemented anywhere in the supply chain. In the second case, the regular stochastic programming formulation (2.15)-(2.27) is used and optimized the operational level inspection decisions after a random failure scenario is realized.

Figures 2.6 and 2.7 clearly show that inspection of goods in the face of failures substantially reduces expected total cost and expected cost of shipped tainted materials. When capacity is equal to the total demand (i.e., capacity $=0 \%$ ), inspection is not instituted under any scenario (see Figures 2.6(a) and 2.6(b)). This is because inspection discards a certain portion of the tainted materials and high cost of unsatisfied demand prevents this when there is no extra capacity. Therefore, inspection is effective only when there is redundant capacity.

As can be seen from Figures 2.7(a) and 2.7(b)), inspection is also effective in reducing costs at different levels of correlation in facility failures.

\subsection{Conclusions}

In this chapter, a two-stage stochastic model was developed to study the problem of supply chain design with unreliable suppliers, correlated failures, and the option to inspect. Computational results suggest that inspection of goods is critical, as it is much cheaper to inspect and discard tainted material than it is to ship damaged 


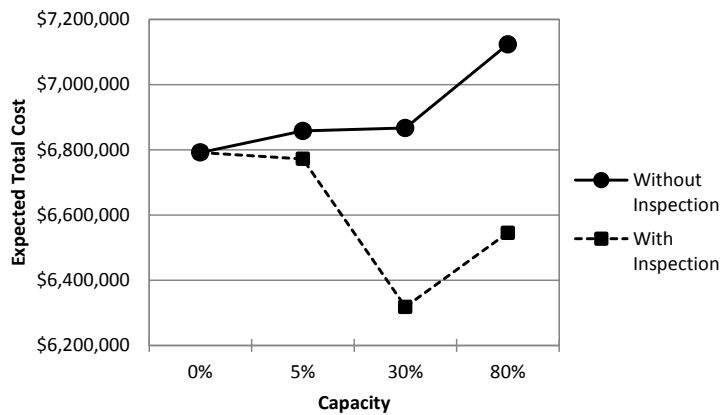

(a) Expected total cost.

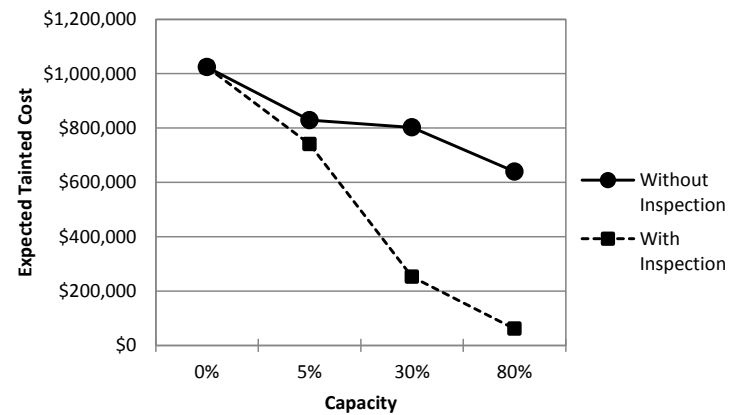

(b) Expected Tainted Cost

Figure 2.6: Effect of inspection option on (a) expected total cost and (b) expected cost of shipped tainted materials. In the experiments, radius of facility circles is set to 9 and the marginal mean vector of facility failures is set to [0.025 0.020 .0150 .060 .050 .015 ] while capacity is varied.

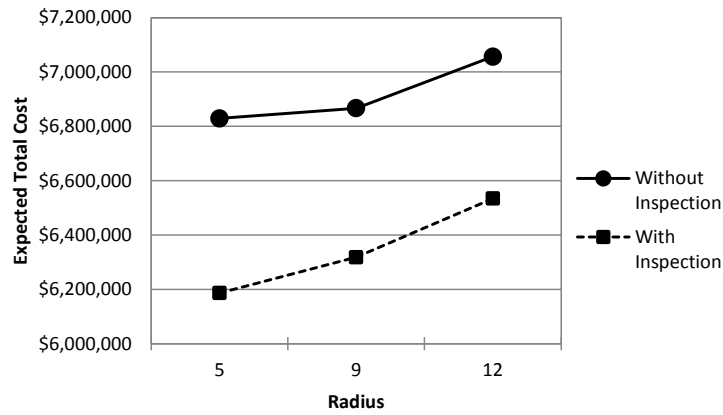

(a) Expected total cost.

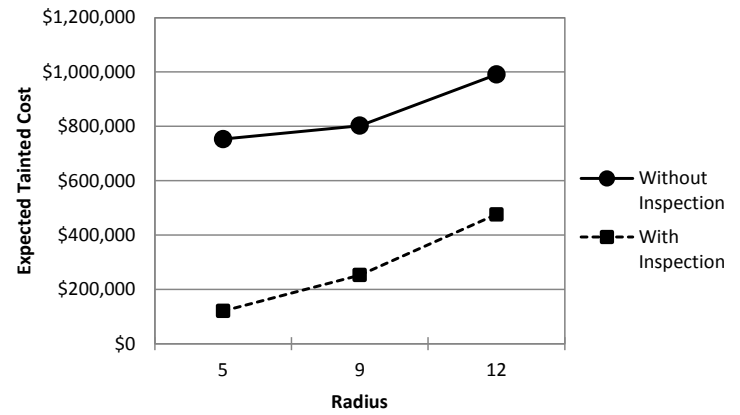

(b) Expected Tainted Cost

Figure 2.7: Effect of inspection on (a) expected total cost and (b) expected cost of shipped tainted materials. In the experiments, capacity is set to $30 \%$ more than the total demand and the marginal mean vector of facility failures is set to [0.025 0.02 0.015 0.06 0.050.015] while radius of facility circles is varied. 
goods to the customer. Further, the results show that when the failure of suppliers is correlated with each other, the total expected cost of supply chain increases relative to cases when the failures are independent. Furthermore, correlated failures can alter the structure of the solutions that are obtained. The numerical experiments show that more facilities are required to hedge against the risk of shortage/supply failure when the correlation is high.

The computational results also indicate that installing redundant capacity is beneficial so that, in the case of disruption, inspection can be instituted to detect and discard a large portion of tainted materials while customers are still able to satisfy their demand. However, redundant capacity can be costly to install - as a result, there is a tradeoff between obtaining enough redundant capacity to recover from supply disruptions, but not too much so that costly capacity goes unused.

In Chapter 3, a multi-objective genetic algorithm will be developed and discussed in details. This algorithm can be easily simplified to handle single objective problem of this chapter for large scale examples. 


\title{
CHAPTER 3. A MULTI-OBJECTIVE APPROACH FOR DESIGNING A ROBUST SUPPLY CHAIN
}

\begin{abstract}
In this chapter, a multi-objective stochastic model is developed to explore the tradeoffs between costs and risk in the supply chain in the event a disruption occurs. Operating cost, unsatisfied cost, tainted and inspection cost are explicitly considered as three conflicting goals to be minimized simultaneously. The first objective (operational cost), minimizes the supply chain configuration cost under normal circumstances without disruptions or failures. The second and third objective minimize the effect of disruption in quantity and quality aspect. Different scenarios for failures of the suppliers are considered and their effect on supplier selection, transportation, unsatisfied demand, tainted materials and inspection strategies is examined. Since this is a NP-hard problem, multiple Pareto optimal solutions are generated by using a genetic algorithm, in which data envelopment analysis is used to calculate the fitness value of supply chain configurations. The proposed hybrid approach removes dominated solutions and yields desirable efficient frontiers.
\end{abstract}

\subsection{Introduction and motivation}

A supply chain is a network of suppliers, production centers, warehouses, and distribution centers. It is through supply chains that raw materials are acquired, transformed, produced, and delivered to the customer [1]. The performance of a supply chain is dependent on the performance of each component in the network, and the ability of those components to be organized and integrated. Due to the complicated interrelations and interactions between a highly diverse set of suppliers, production centers, distribution centers, and customers, supply chains are subject to variety of 
risk factors. Coordination between those entities is typically an important and difficult activity. A relatively small disruption and failure in one component can lead to a massive negative impact across the entire network. Disruption is an unforeseen and often sudden event that brings about damage, loss, and destruction to life and property. Common causes of supply chain disruption include natural disasters, production problems, accidents, labor availability, terrorist attacks, unexpected and sudden shocks, political/economic disasters, and war. Snyder and Daskin [53] listed some actual cases of supply chain disruptions which occurred from 2002 to 2005. For example, in 2005, Hurricane Katrina in the U.S. Gulf Coast region in created a serious impact on all levels of supply chains across different industries and companies. Affected industries included coffee production, oil refining, lumber storage, banana imports, groceries, and retail.

This dissertation is motivated by several real supply chain failures. Heparin, a widely used anticoagulant, is produced from the mucosal tissue of pigs. In 2008, when blue ear pig disease swept through China, tainted heparin was produced and distributed. Contaminated heparin caused 81 deaths and several allergic reactions in the United States and around the world. Thereafter, further testing and product recalls of heparin have occurred on order of the U.S. Food and Drug Administration (FDA) [4]. In addition, in 2008, infant formula was contaminated with melamine in China. China's Administration of Quality Supervision, Inspection and Quarantine (AQSIQ) investigated the issue involving 154 manufacturers. Melamine contamination had been found in $13 \%$ of the sampled manufacturers [54]. Tainted milk impacted over three dozen countries. Thousands of victims (humans, cats, and dogs) fell ill or were killed by the chemical melamine $[55,56]$.

Furthermore, in Japan, the March 11, 2011 earthquake (magnitude 9.0) and subsequent tsunami, nuclear crisis, and infrastructure damage created a significant influence on many companies. Disrupted supply chains affected a broad range of manufac- 
turing industries such as car manufacturing, consumer electronics, and data processing [2]. Automotive companies such as Honda, Toyota, and Nissan had to shut down production upon being faced with lack of component availability. For example, the Toyota factory in Japan (which makes approximately half the vehicles that Toyota sells worldwide [3]) was closed for a while after the earthquake. In another instance, thirty five Nissan suppliers were disrupted by this natural phenomenon. Also, 60\% of the world's silicon wafers are supplied by Japan [2]. Silicon wafers are applied in electronic circuit manufacturing and used in many micro devices. The occurrence of Janpan's earthquake and tsunami led to serious damage in silicon manufacturing. Clearly, lack of necessary materials creates serious issues for global manufacturing.

Motivated by these cases, in this dissertation, two types of supply chain disruptions are considered. The first type of disruption is one that leads to a quality problem with the product (see the heparin or contaminated milk examples above for more clarification). Failure in this context is a function of low-quality raw materials that a facility receives and consequently that facility will produce some materials that are tainted. The second type of disruption is one that affects the quantity (availability) of the product (see the car manufacturing or silicon wafer examples above for more clarification).

Considering these cases, supplier sourcing in two-tiered supply chain is examined. First-tier suppliers are those who produce a product from raw materials. Second-tier suppliers are those who produce the raw materials for the first-tier suppliers. For instance, in the heparin example, pig farmers are second-tier suppliers who produce raw materials for first-tier suppliers, which would include pharmaceutical companies such as Baxter or APP. Here, customers are assumed to be group purchase organizations. "Opening a facility" in this model is equivalent to entering a long-term contractual agreement with a first-tier supplier in order to satisfy the required demand.

Motivated by these cases, this dissertation considers correlated supplier failures. 
For example, in the heparin outbreak, the second-tier suppliers were affected regionally by blue ear pig disease. As a result, more than 12 Chinese first-tier suppliers produced and distributed tainted heparin around the world. A similar correlated failure pattern was seen in other cases. Inclement weather, economic instability, riots, agitation, political unrest, and strikes are other reasons that can cause supplier failure in a region. Previous research $[42,43]$ indicates that correlation among facilities has important impact on the solution and cost. The results presented in Chapter 2 suggest that higher correlation leads to both higher expected total cost and cost of shipped tainted materials. Moreover, correlated failure can modify the structure of the solution.

Motivated by the heparin outbreak and tainted milk cases, this dissertation integrates supply chain design decisions (strategic decisions) with inspection decisions (tactical decisions). For example, in the heparin outbreak, if there had been a proper inspection, tainted heparin would have been detected before reaching customers. Both strategic supply chain design and tactical inspection decision are important to ensure availability and quality in supply chains to mitigate disruption impacts. There is a rich literature $[e . g ., 57,14,15]$ on inspection decisions in supply chain. For example, Rosenkrantz et al. [57] study the placement of inspection stations on networks (such as transportation and communication). They use two different optimization objectives. In the first optimization problem, the objective is to minimize the maximum interval between two successive inspection stations along the path. In the second category, the objective is to minimize the total cost which includes inspection cost and expected penalty cost. A polynomial time algorithm and fully polynomial approximation are presented for analyzing the results.

There are variety of approaches for optimization multi-objective decision-making under uncertainty in supply chain management. For example, Azaron et al. [58] consider several uncertain parameters (such as demands, supplies, processing, transporta- 
tion, shortage and capacity expansion costs) and develop a multi-objective stochastic programming model. The objective is to minimize the variance of the total cost, and the probability of not meeting a certain budget. A goal attainment technique is used to obtain the Pareto-optimal solutions. Guillen et al. [59] construct a two-stage stochastic model to illustrate uncertainty in the production scenario. Three objectives (net present value, demand satisfaction and financial risk) are considered and trade-off between the objectives (Pareto curve) obtained by applying the $\varepsilon$-constraint method. Liu and Nagurney [60] investigate the influence of foreign exchange rate as one of the main uncertainty on supply chain firms who are involved in offshoreoutsourcing activities. They develop a series of simulation to analyze different risk attitudes considering outsourcing and pricing decisions. Franca et al. [61] examine tradeoffs between quality and profit in the multi-objective stochastic modeling framework. A Monte Carlo sampling is used to generate the demand scenarios according to a normal distribution. Financial risk is analyzed considering six sigma measures and finally, a set of Pareto efficient solutions is evaluated. Sabri and Beamon [62] present a multi-objective decision analysis to evaluate cost, customer service levels, and fexibility in supply chain. They consider production, delivery, and demand uncertainty and provide a performance measure to assess network. Matisziw et al. [63] develop a multi-objective linear-integer spatial model to optimize network restoration strategies during disaster recovery. Two objectives (system cost and system flow) are considered and trade-off between the objectives obtained by using the weighting method.

A related branch of literature studies disruption risk in supply chain. For a research overview in this area, the reader is refereed to study Snyder and Daskin [53] and Snyder et al. [64]. Snyder and Daskin [28] develop reliability models based on the P-median problem and fixed charge problem. All facility locations are subject to random disruptions with identical failure probabilities. Models minimize facility location cost and expected transportation cost after failures of facilities. Both mod- 
els are solved using Lagrangian relaxation and a trade-off curve between operating cost and expected failure cost is generated. Qi et al. [65] present a continuous-review inventory problem for a retailer who faces random disruptions both internally and externally. They prove that the cost function is quasi-convex and can therefore be efficiently optimized using standard numerical algorithms. Cui et al. [29] use a mixed integer program formulation and a continuum approximation model to study the reliable uncapacitated fixed charge location problem (RUFL). They use Lagrangian relaxation algorithm and heuristic approach to find near-optimum solutions. Qi et al. [66] consider a nonlinear integer programming model to evaluate the impact of random supply disruptions at the suppliers and retailers side on the retailer location and customer demand allocation decisions. An approximation of the objective function of this model is used to make the model easier to analyze and to solve. Matisziw et al. [67] investigate the sensitivity of network robustness to the network nodes' level of interaction. A mathematical programming model is developed to identify bounds on robustness to arc deletion. Serrano et al. [68] develop a non-dominated sorting genetic algorithm to minimize the impact of supply chain disruption. Disruption is considered by scenarios as unavailabilty of supply side and inaccessibility of path to transport the product. Cost, risk and the place of facilities supporting the supply chain recuperation are considered as different objectives to evaluate algorithm.

The single objective optimization model (2.15)-(2.27) described in Chapter 2 is extend to include a multi-objective two-stage stochastic programming formulation that models disruptions in the supply chain. In Chapter 2, all the expected costs are combined as a single objective to obtain best set of suppliers to use and decide whether or not to inspect their products. In reality, however, the nature of this decision is multi-objective. For ensuring to have a successful result, each objectives need to be considered separately. Therefore in this dissertation, the multi-objective model is solved to obtain an acceptable tradeoff between supply chain configuration 
cost and risk. Operating cost, unsatisfied cost, tainted and inspection cost are explicitly considered as three conflicting goals that have to be minimized simultaneously. Designing a robust supply chain considering all objectives can reduce the significant portion of the costs associated with risk of failure. An effective supply chain not only bring the direct cost of providing customer service down, but it also offer a number of other significant benefits, such as ensuring high quality, high availability, increasing options, and increasing responsiveness.

The proposed model is a multi-objective stochastic mixed-binary model. The model can quickly become intractable for large-scale supply chain since the number of scenarios increases exponentially as the number of candidate facility locations increases. The main problem with the model is having lots of variables, one for each scenario which is computationally intractable by increasing the scales of the problem. Exact solution approaches (that guarantee a provably optimal solution) such as Integer L-Shaped method are not able to solve the single objective version of this problem for more than ten suppliers (see Chapter 2). Approaches based on metaheuristic algorithms, such as Genetic Algorithm (GA), promising alternatives to reduce the high complexity and difficulty of optimization problems under uncertainty. Metaheuristics can search very large spaces of feasible region to find good solutions in a generally smaller computation time. However, there is no guarantee that the solution is optimal [69].

In the solution methodology, multiple Pareto-optimal solutions are generated by using a Genetic Algorithm, in which Data Envelopment Analysis (DEA) is used to calculate the fitness value of supply chain configurations. In the numerical experiments, it is observed that proposed hybrid approach removes dominated solutions and yields desirable efficient frontiers faster than methods based only on GA. A short description of the improved genetic algorithm using data envelopment analysis is described below. 
Genetic Algorithm. A Genetic Algorithm is an iterative algorithm inspired by biological process of natural selection [70]. The search technique starts with a randomly generated population of feasible solutions; each member of the population is equated to a chromosome and represents a possible solution of the optimization problem. In each iteration, the fitness of each chromosome is evaluated with respect to the objective function. For creating the next generation, the current generation will be modified using crossover or mutation operator. The new generation is then a combination of the initial generation and modified chromosomes considering the fact that chromosomes with the higher fitness value have the higher chance of being selected. The iterations will be continued until the termination condition is satisfied.

Data Envelopment Analysis. Data envelopment analysis (DEA) is a powerful non-parametric, linear programming technique to measure the relative efficiency frontier of decision making units (DMUs). The inefficient DMUs are projected onto the best practice frontiers to increase the efficiency of inefficient DMUs [71]. DEA can handle multiple inputs and multiple outputs even with different units. Seiford and Zhu [72] study a DEA model, in the context of undesirable factors. Desirable factors should increase while undesirable factors should decrease. They propose an approach for case where some inputs (outputs) are undesirable and all outputs (inputs) are desirable. Hadi-Vencheh et al. [73] extend the DEA model described in Seiford and Zhu [72] and propose a model considering both of the undesirable factors (inputs/outputs). Bian [74] presents a weighted additive DEA model to evaluate efficiencies without requiring user specified direction vector or any data transformation. Also, their model is designed for any type of desirable/undesirable inputs and outputs.

Integration of $\boldsymbol{G A}$ and $\boldsymbol{D E A}$. The research on combining GA with DEA to solve multi-objective problems is very limited. Yun et al. [75] propose a hybrid GA and DEA method for generating efficient frontiers in multi objective optimiza- 
tion problems. Whittaker et al. [76] develope a hybrid genetic algorithm considering Non-dominated Sorting Genetic Algorithm-II (NSGA-II) for calculating the multiple objective Pareto optimal set and activity analysis (or a proper DEA model) as a local search method. Li et al. [77] propose a two-stage method for solving multiple objective system reliability optimization problems. An improved version of NSGA-II is used to identify the Pareto optimal solutions. Then, a self-organizing map is applied to classify optimal Pareto solutions into several clusters. Also, they use DEA method to reduce the size of Pareto optimal set in each cluster. Wang et al. [78] propose an approach based on GA and DEA to solve multi-stage distribution network expansion planning problems. Lin et al. [79] propose a framework for multi-objective simulation optimization that combine GA and DEA used to evaluate the simulation results.

This dissertation provides a number of important insights and contributions to the literature. First, a multi-objective stochastic model is developed to explore the tradeoffs between costs and risk in the supply chain. It is important to consider these objectives simultaneously to gain a better understanding of disruption effects in the supply chain. Second, this dissertation integrates the supply chain design decision (strategic decision) with inspection decision (tactical decision). Both strategic supply chain design and tactical inspection decision are important to ensure availability and quality in supply chains in order to mitigate disruption impacts. Third, this research considers correlated supplier failures. An approach is adapted to generate correlated binary variables to indicate disruptions, and then, simulate the fractions of tainted products where disruptions occur. Fourth, two types of disruption are considered in the model. The first type of disruption is one that leads to a quality problem of the product (low-quality raw materials that a facility receives). This helps us to develop insights about tainted cost and inspection policy. The quality failures are simulated by considering the supplier's correlation. The disruptions that affect the quantity (availability) of the product are considered to be the second type of 
disruption. This helps us to develop insights about capacity and unsatisfied demand. Finally, a powerful hybrid genetic algorithm is developed, in which data envelopment analysis is used to calculate the fitness value of supply chain configurations to solve the multi-objective stochastic model. In addition, several modifications of the genetic algorithm are proposed. For example, scenario subsampling is considered to evaluate offspring and a simulated annealing approach is used to re-evaluate the acceptance of candidate chromosome. These modifications help to accelerate the GA search process and enhance the search of the feasible region.

The remainder of this Chapter is organized as follows. In Section 3.2, a multiobjective supply chain design model is formulated and a disruption scenario generation approach is discussed. In Section 3.3, a hybrid metaheuristic solution approach is proposed which is based on Data Envelopment Analysis and a Genetic Algorithm. Computational results are presented in Section 3.4. Finally in Section 3.5, the findings are summarized.

\subsection{A Multi-objective supply chain model}

As mentioned previously, the single objective optimization model described in Chapter 2 is extended and a multi-objective optimization model is developed to produce trade-off curves between different types of risks/objectives, with a primary focus on understanding the tradeoff between risk and cost. In this chapter, a supplier sourcing in two-tiered supply chains is considered. First-tier suppliers are those who produce a product from raw materials and second-tier suppliers are those who produce the raw materials for the first-tier suppliers. Also, customers are assumed to be group purchase organizations and "opening a facility" in equates to entering into a longterm contractual agreement with a first-tier supplier. The model determines which subset of a set of candidate locations $L$ should be opened to satisfy the demand of a set of customers in the event of a disruption. It is assumed that each of the selected 
locations can fail and multiple failures may occur simultaneously.

Two types of disruption are considered: failures that affect the quantity of the products and failures that affect the quality of the products. The quality failures are simulated by considering the suppliers' correlation. Also, it is assumed that once a supplier fails, it can still continue supplying products with an additional cost of safety tests/quality control measures. Details of the scenario generation process for disruption are described in Section 3.2.2.

Moreover, the option to inspect is integrated in the model. Disruption directly affects the quality of products. The quality of products can be improved by considering inspection of the units received from the suppliers. Especially in cases such as pharmaceutical supply chains, inspection is critical in ensuring that only high-quality drugs are distributed to patients.

"Operating cost", "unsatisfied cost", and "tainted and inspection cost" are explicitly considered as three conflicting goals that have to be minimized simultaneously. The first objective (operational cost) minimizes the supply chain configuration cost under normal circumstances without disruptions or failures. While, the second and third objectives attempt to minimize the effect of disruption in quantity and quality aspect. It is important to consider these objectives simultaneously to gain a better understanding of disruption in the supply chain.

It is assumed that if demand of customer $i$ is not satisfied, a per unit penalty $u_{i}$ is assessed for the unmet demand of customer $i$. In some cases, it may be less expensive to pay a penalty than to assign a customer to the facility. This is modeled using a dummy "emergency facility", $m$, which has no fixed cost to be opened and never fails. Note that the emergency facility $m$ is included in the set of candidate locations $L$. The transportation cost $d_{i m}$ is simply the penalty for unsatisfied demand, $u_{i}$.

\section{Model notation:}

- $I$ is the set of customers $(i \in I)$. 
- $L$ is the set of candidate facility locations $(l \in L)$. Included in the set $L$ is a dummy facility indexed by $m$ (with no fixed cost: $f_{m}=0$ ) which can satisfy all demand.

- $f_{l}$ is the fixed cost of opening a facility at candidate location $l$.

- $c_{l}$ is the capacity at candidate location $l$.

- $n_{l}$ is the fixed cost of inspection at candidate location $l$.

- $b_{i}$ is the total demand of customer $i$.

- $d_{i l}$ is the per unit cost of satisfying demand from customer $i$ from location $l$.

- $u_{i}$ is the per unit cost of customer $i$ 's unsatisfied demand (i.e., $d_{i m}=u_{i}$ ).

- $t_{i}$ is the cost per unit of "tainted" material shipped to customer $i$.

- $S$ is the set of scenarios, indexed by $s$. A scenario is defined as an event where facilities that belong in a subset $J \subset L$ candidate facility locations have failed, and facilities that belong in the set $L \backslash J$ have not failed.

$-p_{s}$ is the probability of scenario $s$ occurring.

- $q_{l s}$ represents the fraction of tainted material produced at facility $l$ under scenario $s$ (one can also consider this as the probability that an item produced is tainted). Note that $q_{m s}=0$, i.e., the dummy facility $m$ never fails.

- $r_{l s}$ represents the fraction of tainted material produced at facility $l$ under scenario $s$ after inspection, i.e., the proportion of tainted material that is not detected even after inspection and still reaches the customer. Note that if inspection is used $100\left(q_{l s}-r_{l s}\right) \%$ of material produced is discarded. 
- $h_{l s}$ represents the fraction of available material produced at facility $l$ under scenario $s$, i.e., based on some other kind of disruption, some proportion of material will not be available.

\section{Decision variables in the model:}

- $x_{l}= \begin{cases}1, & \text { if a facility is opened at candidate facility location } l, \\ 0, & \text { otherwise. }\end{cases}$

- $y_{i l s} \in[0,1]$ is the proportion of production capacity at facility $l$ assigned to customer $i$ under scenario $s$.

- $w_{i l s} \in[0,1]$ is the proportion of demand of customer $i$ satisfied by facility $l$ under scenario $s$.

- $v_{i l s} \in[0,1]$ is the tainted proportion of the demand of customer $i$ supplied by facility $l$ under scenario $s$.

- $z_{l s}= \begin{cases}1, & \text { if inspection is used at facility } l \text { under scenario } s \\ 0, & \text { otherwise. }\end{cases}$

\subsubsection{Mathematical formulation of the model:}

In this section, a multi-objective supply chain model with unreliable supply sources is presented. To provide insights into the tradeoffs between different types of risks/costs, three objectives are defined as below:

1. Operating cost $\left(g_{1}\right)$ : Let $g_{1}$ be the operating cost under normal circumstances. In other words, $g_{1}$ minimizes supply chain configuration cost when all facilities are working normally without disruptions or failures, which includes the fixed cost of opening facilities and the cost of transportation. Also, no dummy facility 
and scenario index exist.

$$
\min g_{1}=\sum_{l \in L} f_{l} x_{l}+\sum_{i \in I} \sum_{l \in L} b_{i} d_{i l} w_{i l}
$$

2. Unsatisfied cost $\left(g_{2}\right)$ : Let $g_{2}$ be the expected cost of unsatisfied demand when some disruption or failure occurs. In other words, $g_{2}$ minimizes disruption cost that affects the quantity (availability) of the products. Note that the decision variables are indexed by a scenario index and also, the dummy facility is available to use.

$$
\min g_{2}=\sum_{s \in S} p_{s}\left(\sum_{i \in I} b_{i} u_{i} w_{i m s}\right)
$$

After optimizing the second objective, the result of $w_{i m s}$ (the proportion of demand of customer $i$ satisfied by dummy facility under scenario $s$ ) will be used as a parameter for the third objective.

3. The summation of tainted and inspection cost $\left(g_{3}\right)$ : Let $g_{3}$ be the expected cost of tainted material and inspection. In other words, $g_{3}$ minimizes disruption costs that affect the quality of the products when dummy facility decisions $\left(w_{i m s}\right)$ are fixed based on the second objective results.

$$
\min g_{3}=\sum_{s \in S} p_{s}\left(\sum_{i \in I} \sum_{l \in L} b_{i} t_{i} v_{i l s}+\sum_{l \in L} n_{l} z_{l s}\right)
$$

The constraints in the model are as follows:

1. Dummy facility constraint: the Constraint (3.4) refers to availability of the dummy facility with infinite capacity in case of disruption.

$$
x_{m}=1
$$


2. Capacity constraint: Constraint (3.5) prevents demand from being assigned to a facility unless the facility is opened. Besides, it ensures that the total customer demand $\left(b_{i}\right)$ assigned to any facility $(l)$ cannot exceed the facility's capacity.

$$
\sum_{i \in I} y_{i l s} \leq x_{l}, \quad \forall l \in L, s \in S
$$

3. Demand constraint: Constraint (3.6) requires that the entire demand of every customer is satisfied. Note that in case of any disruption, a portion of customer demand may be satisfied by the dummy facility $m$.

$$
\sum_{l \in L} w_{i l s}=1, \quad \forall i \in I, s \in S
$$

4. Shipping constraints: Constraints (3.7) and (3.8) track the amount of product that is shipped to customer $i$ from facility $l$ to satisfy a customer's demand. In particular, if inspection is not implemented, $z_{l s}=0$ and Constraint (3.8) becomes inactive. In this case, Constraint (3.7) becomes binding and ensures that the amount shipped is the proportion of facility l's capacity dedicated to customer $i$. However, if inspection is implemented, $z_{l s}=1$ and Constraint (3.8) becomes binding. This will adjust the amount of product shipped to account for the tainted product that is caught in inspection and discarded.

$$
\begin{aligned}
& b_{i} w_{i l s} \leq c_{l} h_{l s} y_{i l s}, \quad \forall i \in I, l \in L, s \in S \\
& b_{i} w_{i l s} \leq\left(1-\left(q_{l s}-r_{l s}\right)\right) c_{l} h_{l s} y_{i l s}+c_{l} h_{l s}\left(1-z_{l s}\right), \quad \forall i \in I, l \in L, s \in S
\end{aligned}
$$

5. Tainted-capacity constraints: Constraints (3.9) and (3.10) together calculate the amount of tainted material that is shipped to the customer. Without inspection, Constraint (3.9) implies that all of the tainted goods will reach the customer. 
With inspection, Constraint (3.10) implies that only material passing inspection is shipped to the customer.

$$
\begin{aligned}
& b_{i} v_{i l s} \leq q_{l s} c_{l} h_{l s} y_{i l s}, \quad \forall i \in I, l \in L, s \in S \\
& b_{i} v_{i l s} \leq r_{l s} c_{l} h_{l s} y_{i l s}+q_{l s} c_{l} h_{l s}\left(1-z_{l s}\right), \quad \forall i \in I, l \in L, s \in S
\end{aligned}
$$

6. Tainted-demand constraints: Constraints (3.11) and (3.12) together track the amount of tainted material that is shipped to a customer. Without inspection, Constraint (3.11) becomes binding while with inspection, Constraint (3.12) becomes binding.

$$
\begin{aligned}
& v_{i l s} \geq q_{l s} w_{i l s}-z_{l s}, \quad \forall i \in I, l \in L, s \in S \\
& v_{i l s} \geq r_{l s} w_{i l s}-\left(1-z_{l s}\right), \quad \forall i \in I, \quad l \in L, s \in S
\end{aligned}
$$

7. Non-negativity and binary constraints: Constraints (3.13), (3.14) and (3.15) denote the decision variables (continuous and binary) in the model.

$$
\begin{aligned}
& y_{i l s}, w_{i l s}, v_{i l s} \in[0,1], \quad \forall i \in I, l \in L, s \in S \\
& z_{l s} \in\{0,1\}, \quad \forall l \in L, \quad s \in S \\
& x_{l} \in\{0,1\}, \quad \forall l \in L
\end{aligned}
$$

\subsubsection{Disruption scenario generation}

As mentioned earlier, two types of disruption are considered in the model. The first type of disruption is one that leads to a quality problem of the product. Failure in this context is a function of tainted raw materials that a facility receives. In other words, failure of facilities is not complete (absolute) and a facility can be functioning, producing some materials that are good and some that are tainted. This is an 
important point to take into account when the second-tier suppliers are the cause of failures. Therefore, an approach for generating correlated binary variables to indicate disruptions is proposed, and then, the fractions of tainted products where disruptions occur are simulated. In this dissertation, the Qaqish [45] method for generating $L$ binary correlated variables from a given marginal mean vector and correlation matrix is used. The mechanism proposed by Qaqish [45] is computationally efficient for large $L$, while simultaneously permitting unpatterned correlation and non-stationary data. The scenario generation computational procedure for $q_{l s}, r_{l s}$ are implemented as follows.

Step 1: Data preparation

- correlation matrix and marginal mean vector (long-run percentage of tainted production by facilities) are created based on historical data or expert knowledge.

Step 2: conditional probabilities are calculated by following Qaqish [45].

Step 3: scenario generation

- correlated binary variables (representing facility failures) are simulated.

- $q_{l s}$ is simulated by using the cumulative density function (CDF) of tainted production.

- $r_{l s}$ is calculated by $r_{l s}=\mathcal{F}\left(q_{l s}\right)$ where function $\mathcal{F}\left(q_{l s}\right)$ needs to be adjusted based on particular characteristic of applications.

For details of correlated scenario generation failures, the reader is referrd to the Section 2.2.3.

Disruptions that affect the quantity (availability) of the product are considered to be the second type of disruption. It is assumed that some suppliers may lose their ability to supply, such as oil suppliers in the Middle East. This help us to generate 
insights about capacity and unsatisfied demand. In this dissertation, it is assumed that the fraction of available material produced at facility $l$ under scenario $s\left(h_{l s}\right)$ is uniformly distributed over the range $[0.8,1]$.

\subsection{Solution approach}

In the proposed solution methodology, multiple Pareto-optimal solutions are generated by using the GA, in which DEA is used to calculate the fitness value of supply chain configurations. In addition, several modifications in the genetic algorithm are applied. For example, scenario subsampling is considered to evaluate offspring and also simulated annealing approach is used to re-evaluate the acceptance of candidate chromosome. These modifications help to accelerate the GA search process and also extend the visited areas of feasible region. Also, the same experiment is repeated for regular GA-DEA ignoring mentioned modifications (i.e., ignoring scenario subsampling and simulated annealing approach) in order to compare their relative performance. A short description of the improved genetic algorithm using data envelopment analysis is given below.

\subsubsection{Data Envelopment Analysis.}

In this dissertation, the relative efficiency scores are used as a fitness function in the GA process. One input $\left(g_{1}\right)$ and two undesirable outputs $\left(g_{2}, g_{3}\right)$ are considered. The DEA models presented in $[72,73,74,80]$ were investigated for both the CCRInput-oriented version and BCC-Output-oriented version. The CCR-Input-oriented version (as is shown below) of the model presented by Seiford and Zhu [72] provides best improvement in the discriminatory power of DEA and helps differentiating truly

efficient design points from inefficient ones. Therefore, here the DEA model is as 
below.

$$
\begin{aligned}
& \min \theta_{o} \\
& \sum_{l \in L} \lambda_{l} \cdot g_{1, l} \leq \theta_{o} \cdot g_{1, o}, \\
& \sum_{l \in L} \lambda_{l} \cdot\left(-g_{2, l}+\gamma_{1}\right) \geq-g_{2, o}+\gamma_{1}, \\
& \sum_{l \in L} \lambda_{l} \cdot\left(-g_{3, l}+\gamma_{2}\right) \geq-g_{3, o}+\gamma_{2}, \\
& \lambda_{l} \geq 0, \quad l=1,2, \ldots, L .
\end{aligned}
$$

where $\theta_{o}, g_{1, o}, g_{2, o}, g_{3, o}$ represent the relative efficiency score, the input, and the two output vectors of $\mathrm{DMU}_{o}$ under evaluation. It is assumed that

$\gamma_{1}=1+\max \left\{g_{2,1}, g_{2,2}, \ldots, g_{2, L}\right\}, \gamma_{2}=1+\max \left\{g_{3,1}, g_{3,2}, \ldots, g_{3, L}\right\}$ to satisfy the conditions required by the method.

In the case that the problem includes only one objective, instead of using DEA, the actual value of the objective can be used as the fitness value.

\subsubsection{Hybrid Genetic Algorithm.}

The proposed hybrid algorithm is an effective way to provide a set of most preferred alternatives. This way, the decision-maker needs to focus only on limited non-dominated solutions for the final choice. The general framework of the proposed

hybrid GA is illustrated in Figure 3.1. Details of the proposed GA framework are given below. 


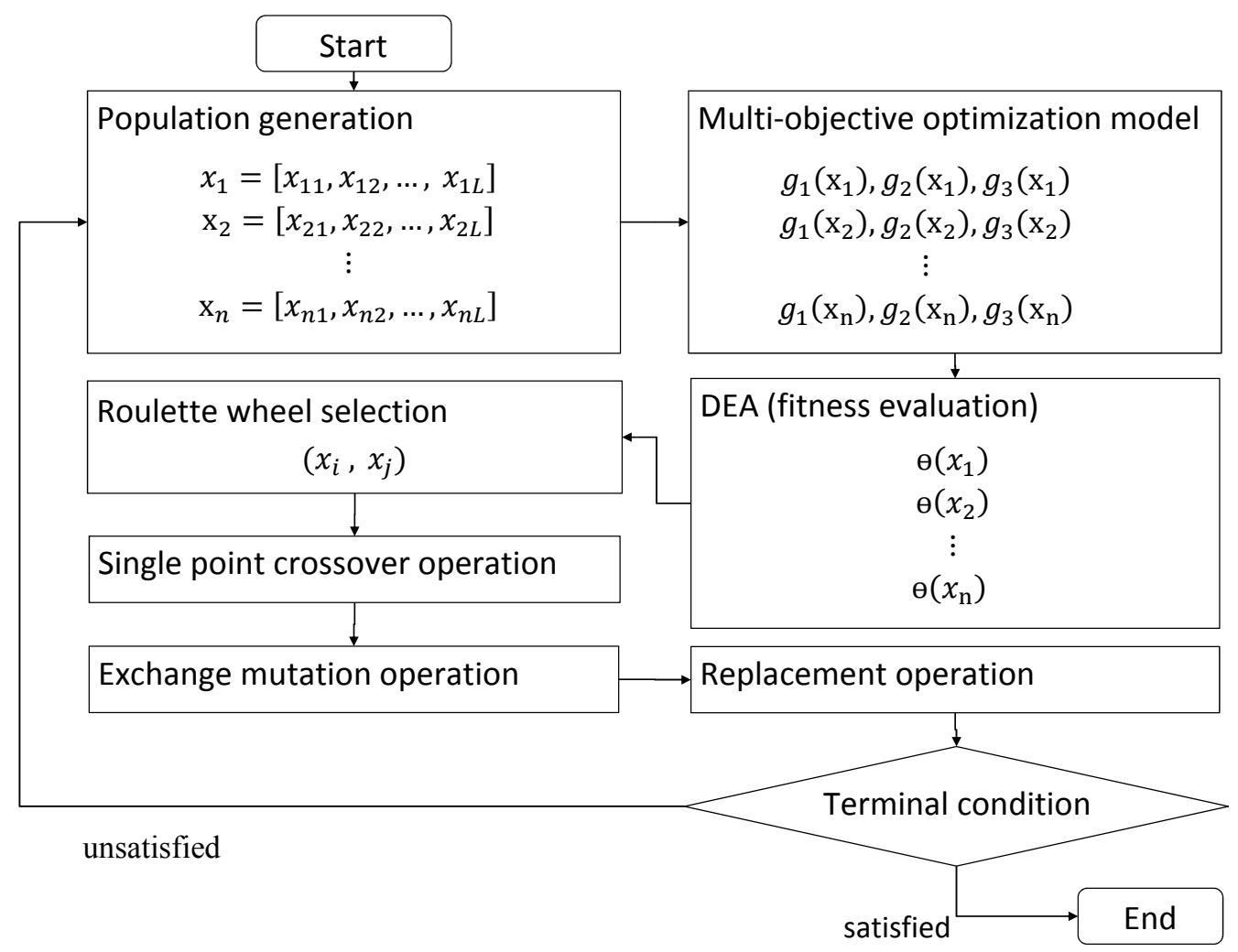

Figure 3.1: Framework for hybrid genetic algorithm.

Chromosome representation. Each chromosome represents a potential supply chain configuration (i.e., the locations of the open facilities). A chromosome is composed of genes. Here, each gene has a binary value which represents whether the facility is chosen to be open or not. The chromosome length is equal to the total number of potential facilities $(L)$ and the location of a gene indicates the number of the facility $(l \in L)$. An example of a chromosome is illustrated in Figure 3.2. Based on the definition of the first objective, the supply chain configuration should satisfy demand under normal circumstances. Therefore, each chromosome needs to satisfy the capacity constraint to make sure that current configuration has the ability to establish a supply chain.

$$
x_{1}=\left[x_{11}, x_{12}, x_{13}, x_{14}, x_{15}, x_{16}, \ldots, x_{1 L}\right]
$$

\begin{tabular}{l|l|l|l|l|l|l|l|}
\hline 1 & 1 & 0 & 0 & 1 & 0 & $\ldots$ & 1 \\
\hline
\end{tabular}

Figure 3.2: An example of chromosome representation 
Initial population. Out of $2^{L}$ possible combination of chromosomes, a pool of $n$ feasible chromosomes is generated randomly as an initial population.

Fitness function. The fitness value evaluates the chromosome structure and returns the relative strength of a chromosome. Chromosomes with larger fitness value are stronger and more desirable. For a given population $\left(x_{1}, x_{2}, \ldots, x_{n}\right)$, the value of $\left\{g_{1}\left(x_{1}\right), \ldots, g_{1}\left(x_{n}\right)\right\},\left\{g_{2}\left(x_{1}\right), g_{2}\left(x_{2}\right), \ldots, g_{2}\left(x_{n}\right)\right\},\left\{g_{3}\left(x_{1}\right), g_{3}\left(x_{2}\right), \ldots, g_{3}\left(x_{n}\right), g_{3}\left(x_{n+1}\right)\right\}$ are calculated and used as DMU's input and outputs in the DEA model. The relative efficiency score $\left(\theta\left(x_{l}\right)\right)$ that is calculated by using the DEA model, will be used as a fitness value for each $x_{l}$. Also for calculating $\theta\left(x_{l}\right)$ in the DEA model, all the efficient frontier points in previous iterations are considered to improve the discriminatory power within DEA. In other words, the global and local impacts are simultaneously modeled to differentiate truly efficient design from inefficient ones.

Selection. The selection operator is selecting the parent (mom and dad) chromosomes for the next crossover process. In this research, the roulette wheel selection method is used to select the parents according to their fitness value. The chromosomes with higher fitness values therefore have a greater chance to be selected.

Crossover operation. The crossover operator is assembling the new chromosome (offspring) from a given parent's chromosome. A single point crossover is used to generate new solutions.

Mutation operation. The purpose of mutation operations is to increase the diversity of the population to avoid solution process from falling into local optimum. The exchange mutation is used to promote diversity of solutions.

Replacement operation. The replacement operation is used to generate a new population. In this dissert-ation scenario sub-sampling is used to evaluate offspring. A simulated annealing approach is used to re-evaluate the acceptance of candidate chromosome. These modifications help to accelerate the GA search process and also extend the areas of feasible region searched. The replacement operation is imple- 
mented as follows given a candidate chromosome $\left(x_{n+1}\right)$.

Step 1: candidate chromosome $\left(x_{n+1}\right)$ has to be distinct from the current population (in iteration $\mathrm{j}$ ). If the condition is satisfied, go to the next step; otherwise, regenerate a new candidate chromosome.

Step 2: for a given candidate chromosome $\left(x_{n+1}\right)$, calculate the values of objectives function $\left(g_{1}\left(x_{n+1}\right), g_{2}\left(x_{n+1}\right), g_{3}\left(x_{n+1}\right)\right)$. In this regard, the scenario sub-sampling method is used to evaluate offspring and decrease computing time. In other words, instead of optimizing the model for all possible scenarios $(|S|)$, the model is optimized by considering some proportion of scenarios. In this way, a quick estimate of the performance of candidate chromosome can be computed.

Step 3: recalculate the fitness values of the current population and new candidate chromosome $\left(x_{1}, x_{2}, \ldots, x_{n}, x_{n+1}\right)$ by using DEA. Assume that $\theta\left(x_{1}\right), \theta\left(x_{2}\right), \ldots$, $\theta\left(x_{n}\right), \theta\left(x_{n+1}\right)$ are new relative efficiency scores (fitness values).

Step 4: compare the $\min \left\{\theta\left(x_{1}\right), \theta\left(x_{2}\right), \ldots, \theta\left(x_{n}\right)\right\}$ and $\theta\left(x_{n+1}\right)$.

- if $\theta\left(x_{n+1}\right) \leq \min \left\{\theta\left(x_{1}\right), \theta\left(x_{2}\right), \ldots, \theta\left(x_{n}\right)\right\}$, then, move to Step 5 .

- otherwise, move to Step 6.

Step 5: use simulated annealing cooling approach to re-evaluate the acceptance of candidate chromosomes. In this way, the candidate chromosome has a greater chance to be part of the new population, which ultimately reduces the computational time. In this step

- set an initial temperature $T$ and a reduction factor $r, 0<r<1$.

- calculate the acceptance probability $(\eta)$. The current solution $x_{n+1}$ with probability $\eta=e^{\frac{\theta\left(x_{n+1}\right)-\min \left\{\theta\left(x_{1}\right), \theta\left(x_{2}\right), \ldots, \theta\left(x_{n}\right)\right\}}{T}}$ is kept. 
- set $T \leftarrow r T$ (it is assumed that the $T$ is same for each generation and reduction factor is implemented after moving to the next generation).

- generate a random number $\in[0,1]$.

- if the random number is bigger than or equal to the $\eta$, ignore this candidate chromosome and regenerate a candidate chromosome and move to Step 1.

- if the random number is less than the $\eta$, move to Step 6.

Step 6: keep the input candidate chromosome as a new candidate for the next population and repeat the previous steps until finding $n$ candidate chromosomes.

Step 7: recalculate the fitness values of the current population $\left(x_{1}, x_{2}, \ldots, x_{n}\right)$ and all the $n$ new candidate chromosomes $\left(x_{n+1}, x_{n+2}, \ldots, x_{2 n}\right)$ by using the DEA model. In this step, assume $\theta\left(x_{1}\right), \theta\left(x_{2}\right), \ldots, \theta\left(x_{n}\right), \theta\left(x_{n+1}\right), \theta\left(x_{n+2}\right) \ldots, \theta\left(x_{2 n}\right)$ are fitness values based on the DEA model.

Step 8: sort $\left\{\theta\left(x_{1}\right), \theta\left(x_{2}\right), \ldots, \theta\left(x_{n}\right), \theta\left(x_{n+1}\right), \theta\left(x_{n+2}\right) \ldots, \theta\left(x_{2 n}\right)\right\}$ and select the top $n$ design points with highest efficiency scores as the next population.

Figure 3.3, shows an example of the replacement operation. 


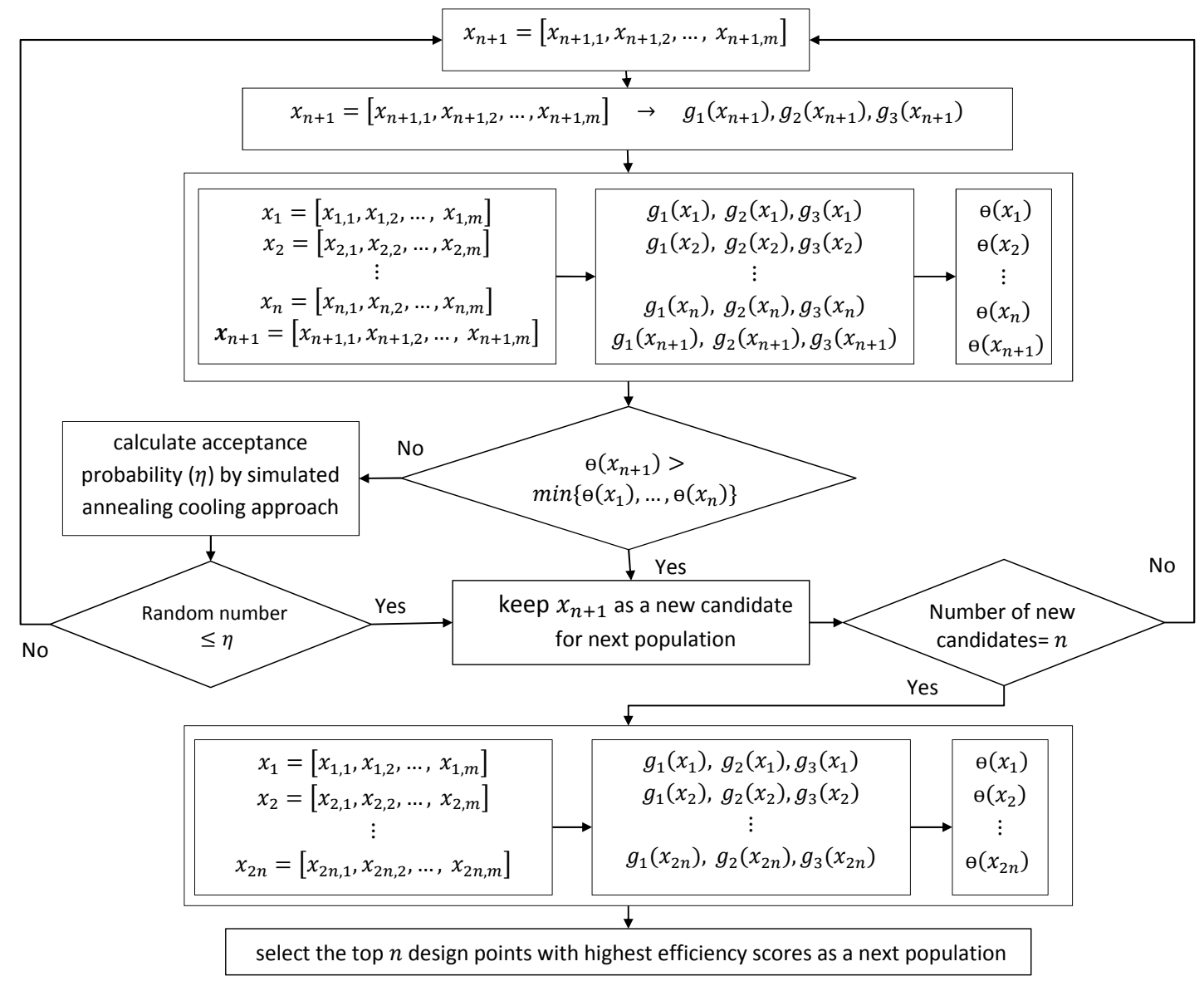

Figure 3.3: An example of the replacement operation.

Terminal condition. All the above-mentioned steps are repeated until a termination condition is reached. A maximum number of generations or a time limit can be used as a stopping criteria. At the end, final solutions are those chromosomes which have the largest fitness values.

\subsection{Numerical Experiments}

In this section, the data used for the experiments and the computational results are summarized. A workstation with two $3.066 \mathrm{GHz}$ Quad-Core Intel Xeon processors and 32 GB memory was used to run all experiments. Both the scenario generation procedure and the improved GA were implemented in the Python programming lan- 
guage. The Gurobi Optimizer 4.5.1 was used to solve all the optimization problem.

\subsubsection{An example of a medium scale problem}

Ten U.S. cities are considered as candidate facility locations (see Table 3.1). All the capitals of all 48 contiguous U.S. states are chosen as customer locations (48 customers).

Table 3.1: Potential facilities

\begin{tabular}{cc|cc}
\hline Potential facilities & Location & Potential facilities & Location \\
\hline F1 & Seattle, WA & F6 & Austin, TX \\
F2 & los Angeles, CA & F7 & St. Louis, MO \\
F3 & Boise, ID & F8 & Chicago, IL \\
F4 & Denver, CO & F9 & Atlanta, GA \\
F5 & Bismarck, ND & F10 & Boston, MA \\
\hline
\end{tabular}

Also, 150 raw material suppliers (second-tier suppliers) are generated randomly in the U.S. map, as shown in Figure 3.4. If a raw material supplier falls within a prespecified distance (circular regions shown in Figure 3.4(a)) from a particular facility, it is assumed that raw material supplier is assigned to that facility. 


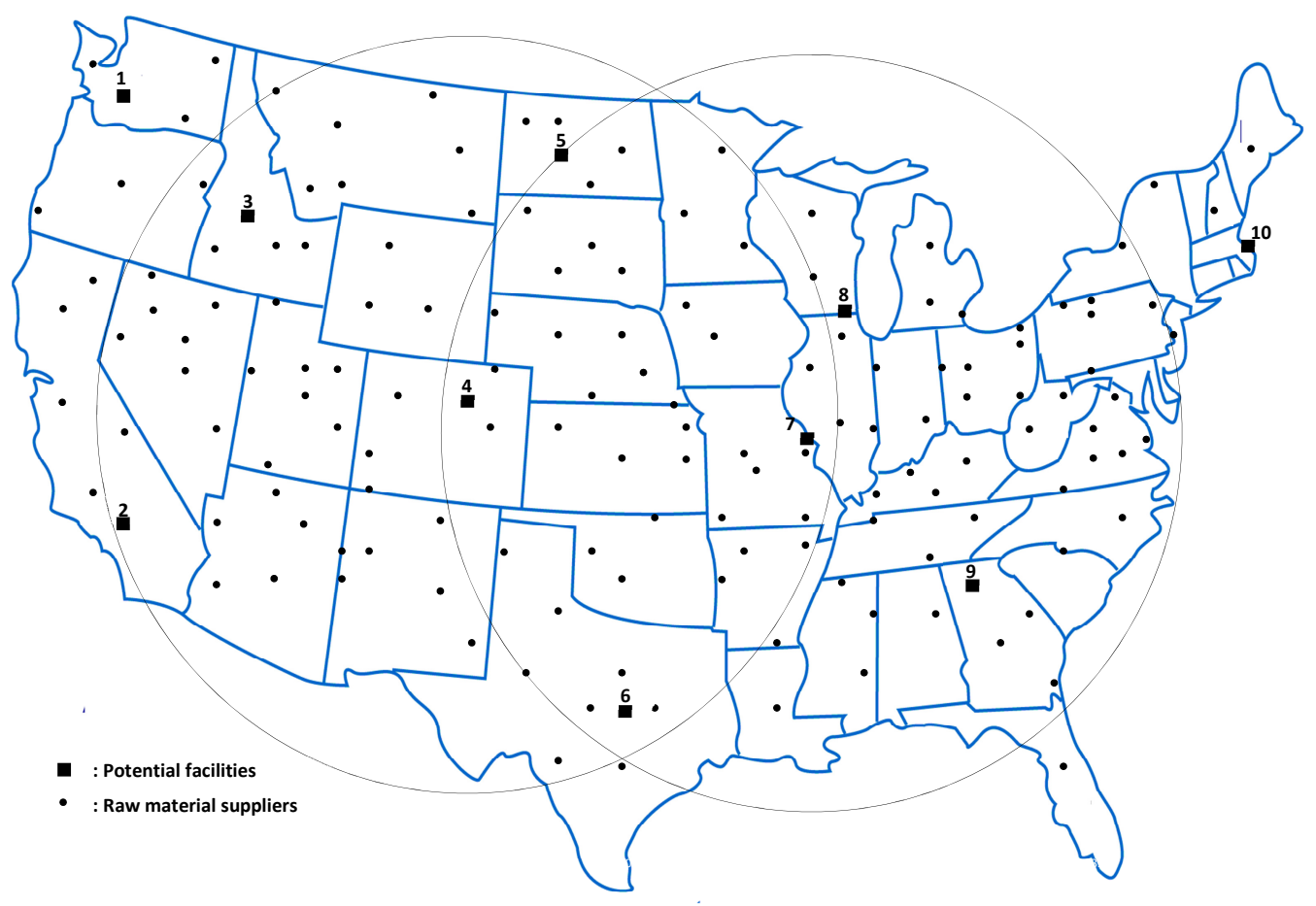

(a) potential facilities and raw material suppliers

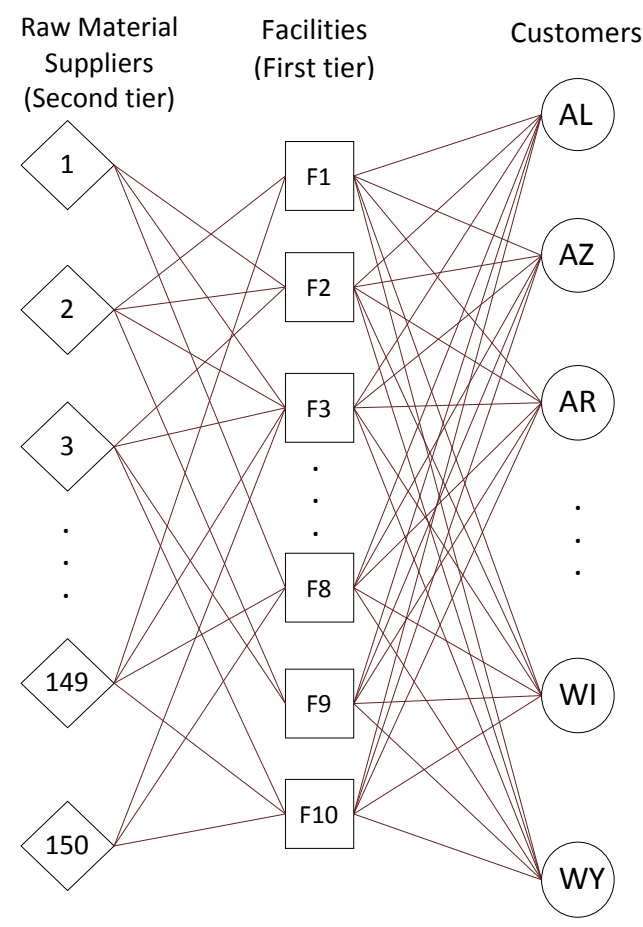

(b) two-tier supply chain

Figure 3.4: Potential facilities and raw material suppliers 
The fixed cost of opening a facility $l$ (or $\left.f_{l}\right)$ is the same for all facilities $\left(f_{l}=\right.$ $\$ 10,000,000)$. The dummy facility has no fixed cost to be opened $\left(f_{m}=0\right)$. The cost of inspection is the same for all facilities $\left(n_{l}=\$ 100,000\right)$. The dummy facility has no inspection cost and never fails $\left(n_{m}=0\right)$. The transportation cost of the facilities (or $d_{i l}$ ), excluding the dummy facility, are normalized over the range $[\$ 100$, \$1000] based on their distance in the U.S. map. The cost of satisfying demand from the dummy facility is equal to the per unit cost of unsatisfied demand (i.e., $\left.d_{i m}=u_{i}\right)$ and assumes to be $\$ 20,000$ for all custmers. The cost per unit of tainted material shipped to customers (or $t_{i}$ ) is $\$ 40,000$ for each customer. The demand of the customers $\left(b_{i}\right)$ are normalized in range $[100,300]$ based on their population. It is assumed that capacity of the facilities ( $c_{l}$, excluding the dummy facility) are all the same $\left(c_{l}=2,145\right)$. Note that the capacity of dummy facility is assumed to be infinite (dummy facility $m$ can satisfy all the demand). The circular regions shown in Figure 3.4(a) are used to determine the raw material suppliers of a facility. The correlation coefficient between two facilities is calculated by considering common raw material suppliers that both facilities work with and the total number of raw material suppliers used by either of the two facilities. For example, the correlation coefficient of $F_{4}, F_{7}$ is calculated as below.

$$
\text { Correlation }\left(F_{4}, F_{7}\right)=\frac{n\left(F_{4} \cap F_{7}\right)}{n\left(F_{4} \cup F_{7}\right)}=\frac{n\left(F_{4} \cap F_{7}\right)}{n\left(F_{4}\right)+n\left(F_{7}\right)-n\left(F_{4} \cap F_{7}\right)}=\frac{40}{86+88-40}=0.299
$$

The result of correlation matrix is shown in Table 3.2.

Table 3.2: Correlation matrix

\begin{tabular}{c|cccccccccc} 
& F1 & F2 & F3 & F4 & F5 & F6 & F7 & F8 & F9 & F10 \\
\hline F1 & 1 & 0.451 & 0.596 & 0.277 & 0.261 & 0 & 0 & 0 & 0 & 0 \\
F2 & 0.451 & 1 & 0.565 & 0.370 & 0.148 & 0.127 & 0 & 0 & 0 & 0 \\
F3 & 0.596 & 0.565 & 1 & 0.505 & 0.410 & 0.082 & 0.058 & 0.077 & 0 & 0 \\
F4 & 0.277 & 0.370 & 0.505 & 1 & 0.562 & 0.364 & 0.299 & 0.280 & 0.134 & 0 \\
F5 & 0.261 & 0.148 & 0.410 & 0.562 & 1 & 0.146 & 0.259 & 0.295 & 0.092 & 0 \\
F6 & 0 & 0.127 & 0.082 & 0.364 & 0.1461 & 1 & 0.356 & 0.282 & 0.292 & 0 \\
F7 & 0 & 0 & 0.058 & 0.299 & 0.259 & 0.356 & 1 & 0.859 & 0.711 & 0.293 \\
F8 & 0 & 0 & 0.077 & 0.280 & 0.295 & 0.282 & 0.859 & 1 & 0.656 & 0.341 \\
F9 & 0 & 0 & 0 & 0.134 & 0.0917 & 0.292 & 0.711 & 0.656 & 1 & 0.386 \\
F10 & 0 & 0 & 0 & 0 & 0 & 0 & 0.293 & 0.341 & 0.386 & 1
\end{tabular}


It is assumed that the marginal mean of a facility (i.e., $\mu_{l}$ : long-run percentage that some fraction of its production will be tainted) is equal to 0.05 for all facilities. One hundred correlated failure scenarios $(s \in\{1,2, \ldots, 100\})$ are simulated where $p_{s}=0.01$. In addition, $q_{l s}=T_{l s} \times($ corresponding correlated binary variable) where, correlated binary variable is simulated in the scenario generation part and $T_{l s}$ is as below.

$$
T_{l s}= \begin{cases}0.2, & \text { with probability } 0.2 \\ 0.3, & \text { with probability } 0.5 \\ 0.4, & \text { with probability } 0.3\end{cases}
$$

Furthermore, it is assumed that $r_{l s}$ is a linear function of $q_{l s}\left(r_{l s}=0.05 \times q_{l s}\right)$ and $h_{l s}$ is uniformly distributed over the range $[0.8,1]$.

\section{Analysis of the entire feasible solution set}

Experiments for all of the possible supply chain configuration (i.e., each configuration is a binary vector which represents which facilities are chosen to be open or not) were conducted. Knowing the results of all possible feasible solutions assisted in validating and understanding the performance of the proposed hybrid GA. Table 3.3 shows the results for some of the possible supply chain configurations and descriptive statistics. For each possible supply chain configuration, the values of $g_{1}, g_{2}, g_{3}$ are calculated. Also considering all feasible solutions, the global efficiency scores $(\theta)$ are calculated using DEA. As is shown in this particular example, the global efficiency scores vary from 0.0827 to 1 . Also, four supply chain configurations with highest global efficiency score (the efficient frontier solutions) where $\theta=1$ are shown in Table 3.3. 
Table 3.3: Some examples of possible solutions

\begin{tabular}{ccccc}
\hline$x$ & $g_{1}$ & $g_{2}$ & $g_{3}$ & $\theta$ \\
\hline$[0,0,1,1,0,0,1,1,1,1]$ & $61,618,657.0$ & 0.0 & $682,105.9$ & 1 \\
{$[0,0,1,1,0,1,0,1,1,1]$} & $61,621,050.0$ & 0.0 & $286,927.1$ & 1 \\
{$[0,0,1,0,1,1,0,1,1,1]$} & $61,667,077.0$ & 0.0 & $167,161.6$ & 1 \\
{$[1,0,1,0,1,1,0,0,1,1]$} & $61,844,253.0$ & 0.0 & $128,702.6$ & 1 \\
{$[0,0,1,1,0,1,1,0,1,1]$} & $61,621,425.0$ & 0.0 & $544,625.5$ & 0.999968591 \\
{$[1,0,0,1,0,1,0,1,1,1]$} & $61,636,645.0$ & 0.0 & $280,731.6$ & 0.99979 \\
{$[1,0,0,1,0,0,1,1,1,1]$} & $61,634,252.0$ & 0.0 & $703,805.0$ & 0.999746975 \\
{$[1,0,0,1,0,1,1,0,1,1]$} & $61,637,020.0$ & 0.0 & $525,977.4$ & 0.999717417 \\
{$[1,1,0,1,0,0,0,1,1,1]$} & $61,679,146.0$ & 0.0 & $195,614.4$ & 0.999627042 \\
{$[0,1,1,0,0,1,1,0,1,1]$} & $61,685,314.0$ & 0.0 & $180,245.2$ & 0.999622841 \\
$\vdots$ & $\vdots$ & $\vdots$ & $\vdots$ & $\vdots$ \\
{$[1,0,1,1,0,1,0,1,1,1]$} & $71,582,567.0$ & 0.0 & $56,565.1$ & 0.8740 \\
$\vdots$ & $\vdots$ & $\vdots$ & $\vdots$ & $\vdots$ \\
{$[1,1,1,1,1,1,1,1,0,0]$} & $82,210,488.0$ & 0.0 & $54,855.9$ & 0.7612 \\
$\vdots$ & $\vdots$ & $\vdots$ & $\vdots$ & $\vdots$ \\
{$[1,1,1,1,0,1,1,1,1,1]$} & $91,502,401.0$ & 0.0 & $14,413.6$ & 0.6882 \\
$\vdots$ & $\vdots$ & $\vdots$ & $\vdots$ & $\vdots$ \\
{$[1,1,1,1,1,1,1,1,1,1]$} & $101,480,611.0$ & 0.0 & $9,360.8$ & 0.6211 \\
$\vdots$ & $\vdots$ & $\vdots$ & $\vdots$ & $\vdots$ \\
{$[1,1,0,0,1,1,0,0,1,0]$} & $52,865,612.0$ & $3,089,880.0$ & $6,114,880.3$ & 0.137225035 \\
{$[0,1,0,1,1,1,0,0,0,1]$} & $52,351,705.0$ & $3,206,400.0$ & $5,787,996.0$ & 0.112014898 \\
{$[1,1,0,1,1,0,0,0,1,0]$} & $53,026,365.0$ & $3,173,880.0$ & $6,017,124.0$ & 0.1089 \\
{$[1,1,0,1,1,1,0,0,0,0]$} & $53,402,702.0$ & $3,226,740.0$ & $5,907,978.0$ & 0.0908 \\
{$[0,1,0,1,1,1,0,0,1,0]$} & $52,579,375.0$ & $3,325,620.0$ & $5,941,122.0$ & 0.0827 \\
\hline global min & $51,706,865.0$ & 0 & $9,360.8$ & 0.0827 \\
global max & $101,480,611.0$ & $3,502,200.0$ & $6,380,832.0$ & 1.0 \\
global average & $62,000,467.8$ & $1,044,342.0$ & $2,370,255.8$ & 0.6742 \\
\hline & & & &
\end{tabular}

In addition, Figure 3.5 represents two by two comparison between $g_{1}, g_{2}, g_{3}, \theta$. This example clearly shows why a multi-objective approach is important for better trade-off between cost and risk. For example from the data in the Figure 3.5(a), it is apparent that higher $g_{1}$ leads to lower $g_{2}$ and lower $g_{1}$ reaches to higher $g_{2}$. In other words, there is a specific value for $g_{1}$ that for any $g_{1}$ larger than that specific value, $g_{2}$ leads to zero. A similar trend is seen between $g_{1}$ and $g_{3}$ in Figure 3.5(b). As is shown in the Figure $3.5(\mathrm{c}), g_{2}$ and $g_{3}$ go together, so that an increase (or decrease) in $g_{2}$ tends to be associated with an increase (or decrease) in $g_{3}$.

Comparing $g_{1}$ and $\theta$ in Figure 3.5(d) indicates the higher value of $\theta$ is associated with a tight range of $g_{1}$ (in this case between $61.6 \mathrm{M}$ and $63.3 \mathrm{M}$ ). In other words, $\theta$ will 
be reduced if the value of $g_{1}$ is out of this range. Also, opening fewer facilities than mentioned range $\left(g_{1}<61.6 \mathrm{M}\right)$ leads to lowest $\theta$. In addition, opening more facilities than mentioned range $\left(g_{1}>63.3 \mathrm{M}\right)$ is not economical and just leads to higher cost and reduced efficiency trend of the supply chain. However, value of $\theta$ associated with $g_{1}>63.3 \mathrm{M}$, is better than value of $\theta$ associated with $g_{1}<61.6 \mathrm{M}$.

A comparison of $g_{2}$ with $\theta$ in Figure 3.5(e) indicates that in general, solutions with lower $g_{2}$ lead to better $\theta$. However, several solutions with $g_{2}=0$ and different $\theta$ values were found. Thus, further investigation is needed to identify possible cofounding variables that may affect $\theta$.

A comparison of $g_{3}$ and $\theta$ in Figure 3.5(f) indicates the higher value of $\theta$ is associated with a tight range of $g_{3}$ (in this case between $118 \mathrm{~K}$ and $1.4 \mathrm{M}$ ). In other words, $\theta$ will be reduced if the value of $g_{3}$ is out of this range. However, value of $\theta$ associated with $g_{3}<118 \mathrm{~K}$, is better than value of $\theta$ associated with $g_{3}>1.4 \mathrm{M}$. Another important point to consider is that those efficient frontiers don't necessarily containing extreme (minimum or maximum) values of $g_{1}, g_{2}, g_{3}$. 


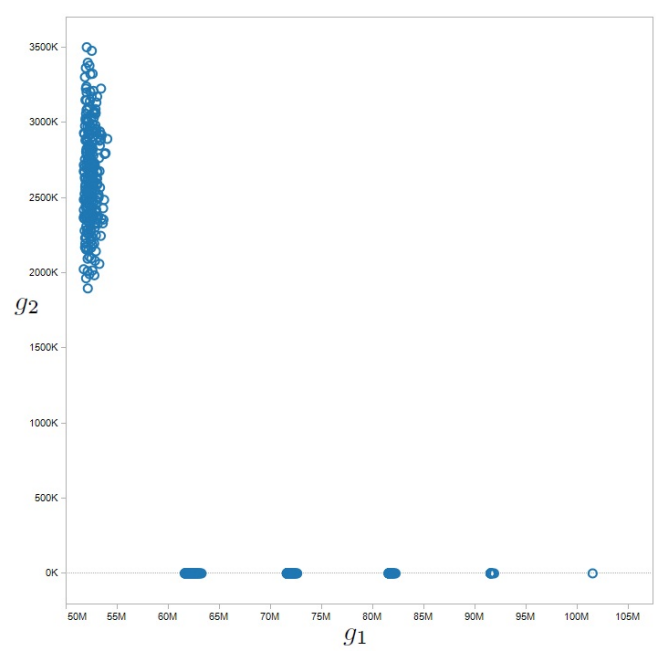

(a) $g_{1}$ vs. $g_{2}$

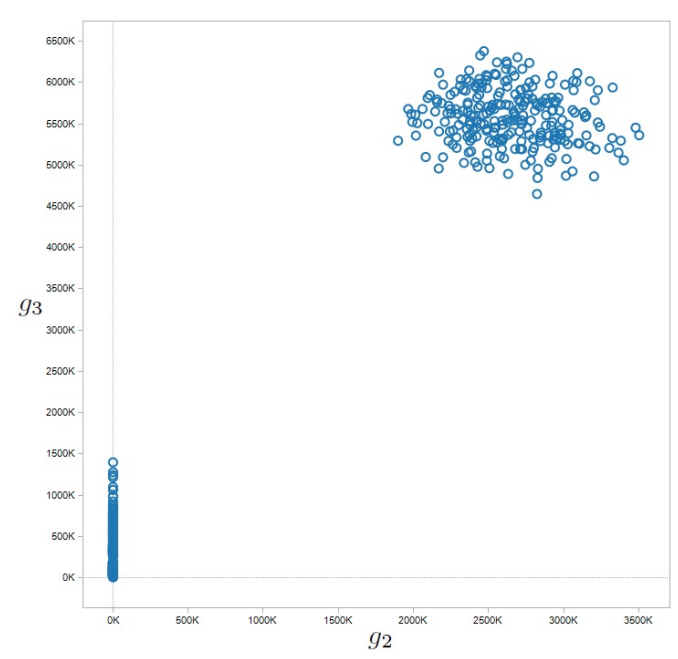

(c) $g_{2}$ vs. $g_{3}$

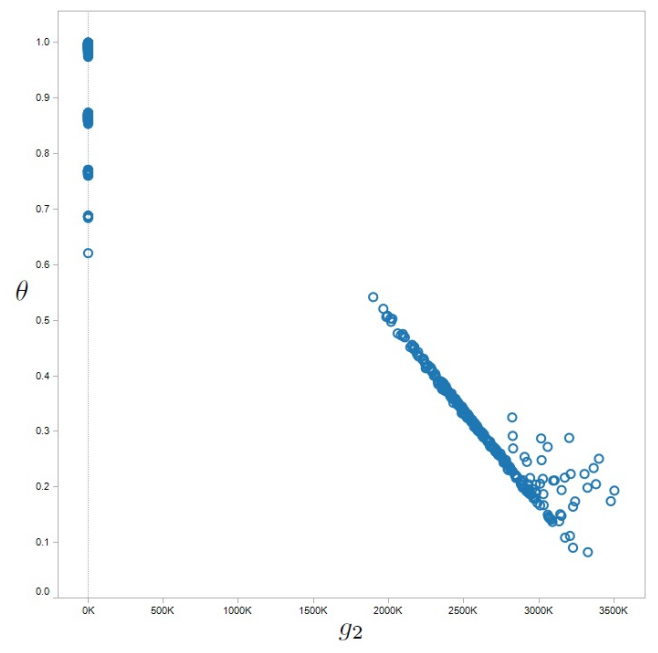

(e) $g_{2}$ vs. $\theta$

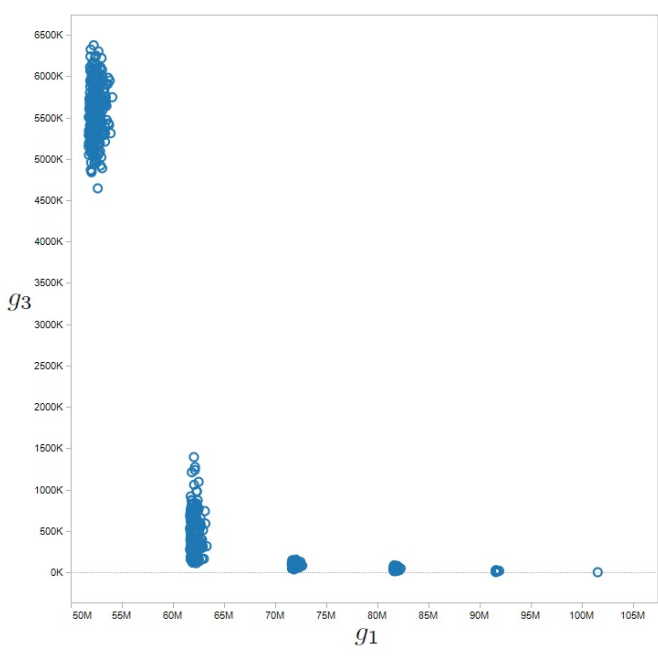

(b) $g_{1}$ vs. $g_{3}$

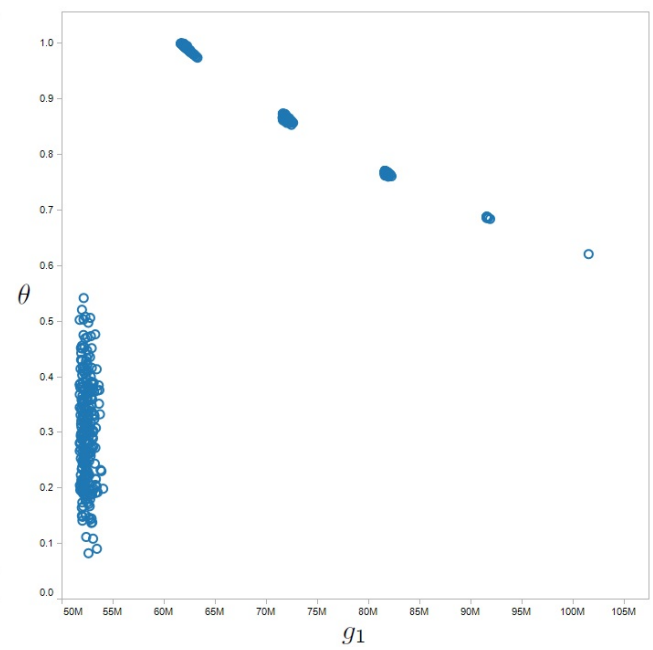

(d) $g_{1}$ vs. $\theta$

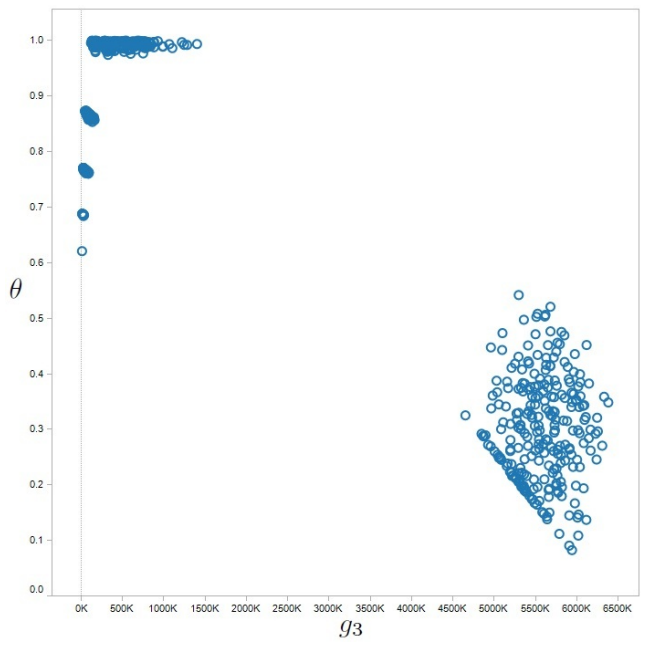

(f) $g_{3}$ vs. $\theta$

Figure 3.5: Two by two comparison between $g_{1}, g_{2}, g_{3}, \theta$. 


\section{Performance and analysis of hybrid GA solutions}

several different GA parameters and time limits are used to evaluate the performance of the proposed solution approach. Table 3.4 summarizes all parameters for config-

uring the GA. Also, the same experiment is repeated for regular GA-DEA, ignoring mentioned modifications (i.e., ignoring scenario sub-sampling and simulated annealing cooling approach) in order to compare their performances. In this dissertation, the first approach is called "Modified GA-DEA", while the second is called "Regular GA-DEA".

Table 3.4: GA parameters

\begin{tabular}{ll}
\hline parameters & values \\
\hline population size & $\{5,10,15\}$ \\
crossover rate & 1 \\
mutation rates & $\{0.1,0.2\}$ \\
scenario subsampling ratio & 0.1 \\
initial temperature & 1 \\
reduction factor & 0.95 \\
running time (sec) & $\{3000,4000,5000,6000\}$ \\
\hline
\end{tabular}

In order to evaluate the performance of both approaches (Modified GA-DEA vs. Regular GA-DEA), the same GA parameters are used. Results of each approach are called local frontier DMUs. In other words, in both approaches, results are the best non-dominated frontier solutions. Since, the value of global efficiency score for each possible feasible solution was already calculated in the section 3.4.1, those values are again used to make a fair comparison of the performance of both approaches. Therefore, for each set of local frontier DMUs, their global efficiency scores and the average of their global efficiency scores (for the given set of local frontier DMUs) are used for final comparison. Figure 3.6 shows a comparison of the two approaches using different GA parameters. 


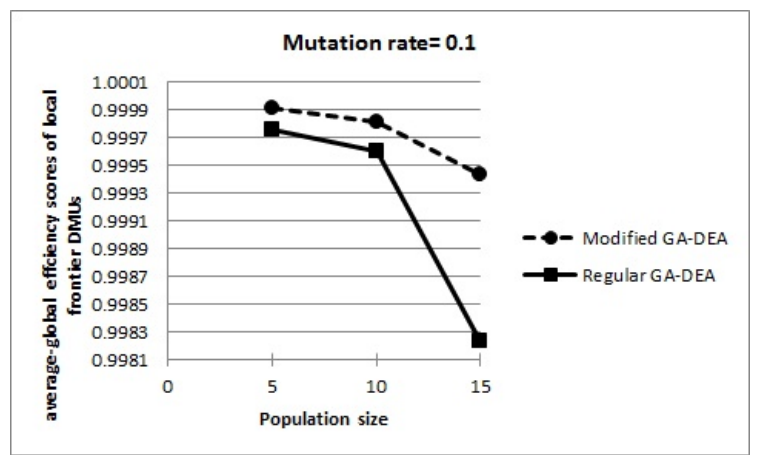

(a) running time $=3000 \mathrm{sec}$

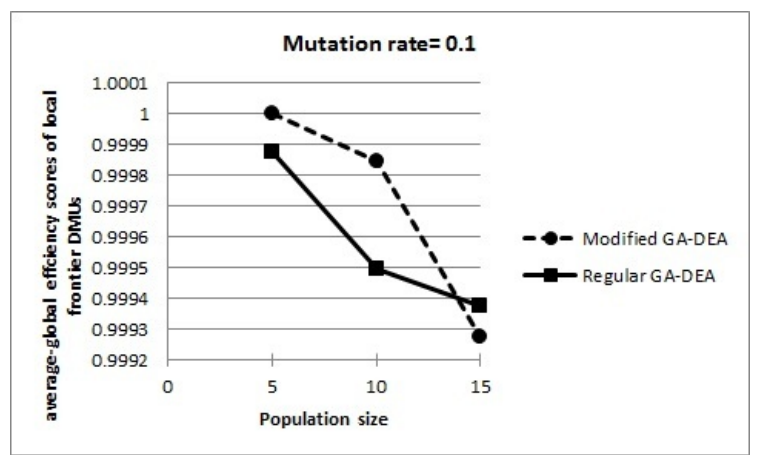

(c) running time $=4000 \mathrm{sec}$

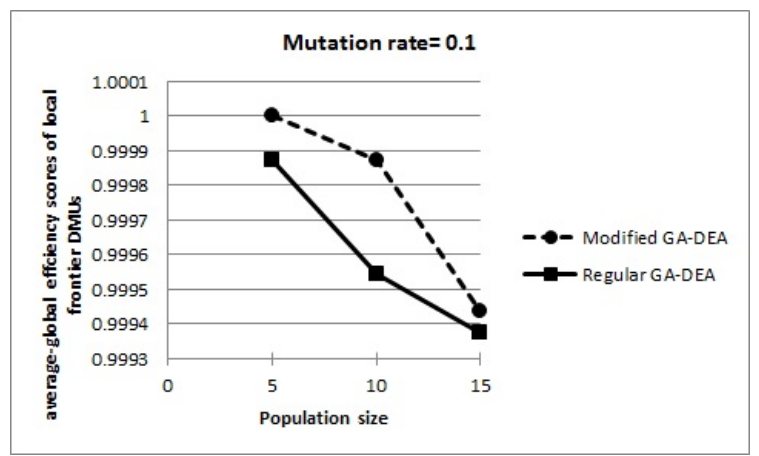

(e) running time $=5000 \mathrm{sec}$

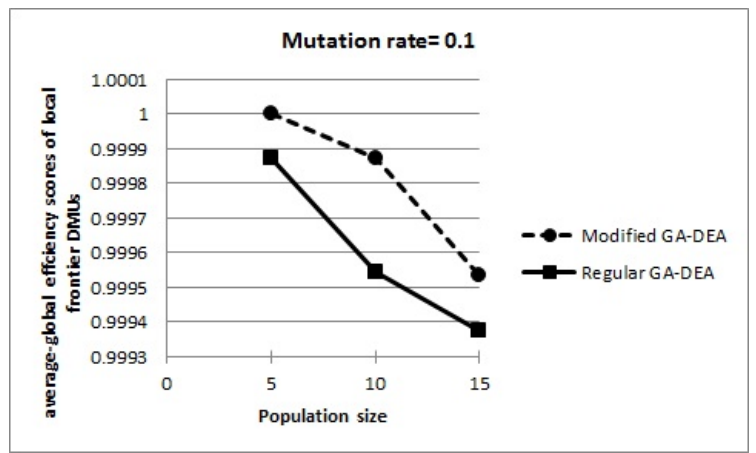

(g) running time $=6000 \mathrm{sec}$

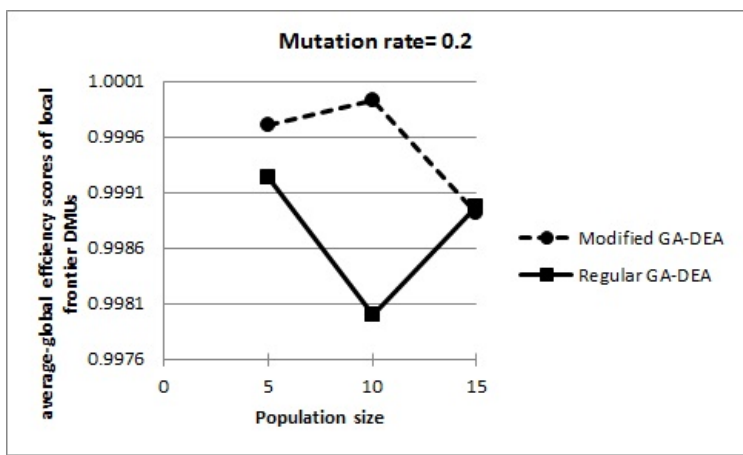

(b) running time $=3000 \mathrm{sec}$

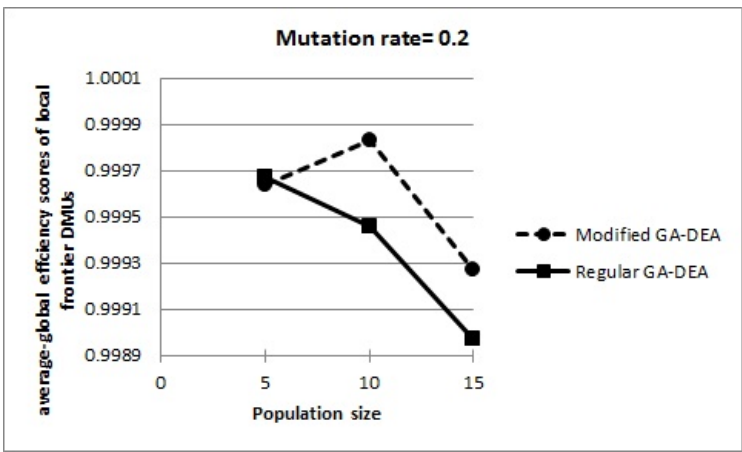

(d) running time $=4000 \mathrm{sec}$

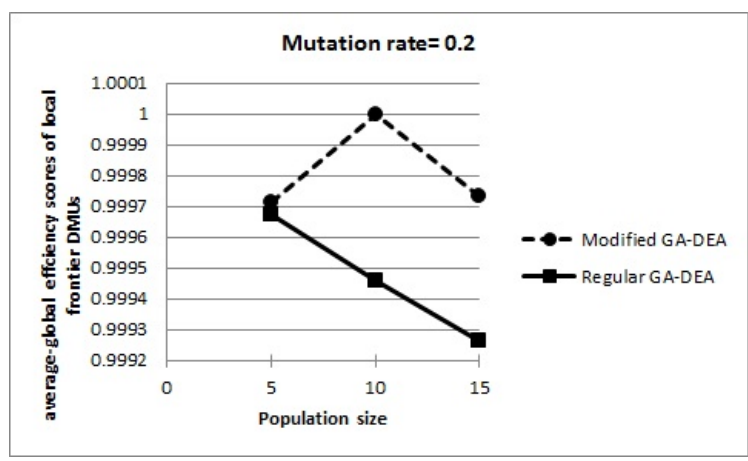

(f) running time $=5000 \mathrm{sec}$

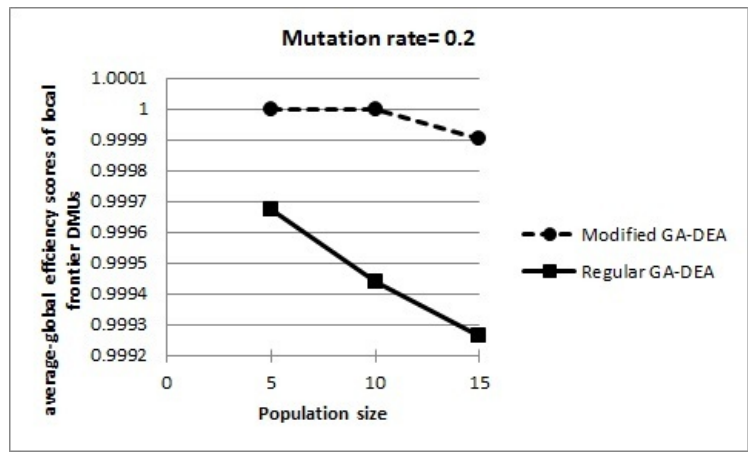

(h) running time $=6000 \mathrm{sec}$

Figure 3.6: Comparison between two approaches 
As mentioned earlier, the Regular GA-DEA does not have scenario sub-sampling and simulated annealing cooling approach while the Modified GA-DEA have them. As it is shown in Figure 3.6, the numerical experiments indicate that the performance of the Modified GA-DEA approach is much better than the Regular GA-DEA. It seems that the Modified GA-DEA version is able to search the space in a more efficient manner. In other words, considering different GA parameters and time limits, the proposed Modified GA-DEA approach can find local frontier DMUs with a higher global efficiency score than Regular GA-DEA.

\subsubsection{An example of a large scale problem}

Next, the scale of the problem is increased by adding more facilities. This is done to gain better insight on the performance of the proposed hybrid GA-DEA to larger application. Twenty U.S. cities are considered as candidate facility locations (see Table 3.5). $2^{20}$ possible supply chain configurations exist, some of which may not be acceptable due to capacity constraints. This dissertation is interested in finding the efficient frontier solutions (or DMUs with higher efficiency scores) without evaluating all $2^{20}$ possible supply chain configurations.

\begin{tabular}{cc|cc|cc|cc}
\multicolumn{7}{c}{ Table 3.5: Potential facilities } \\
\hline $\begin{array}{c}\text { Potential } \\
\text { facilities }\end{array}$ & Location & $\begin{array}{c}\text { Potential } \\
\text { facilities }\end{array}$ & Location & Potential & Location & Potential & Location \\
facilities & \\
\hline F1 & Salem, OR & F6 & Denver, CO & F11 & Des Moines, IA & F16 & Atlanta, GA \\
F2 & Seattle, WA & F7 & Cheyenne, WY & F12 & St. Paul, MN & F17 & Columbus, OH \\
F3 & los Angeles, CA & F8 & Bismarck, ND & F13 & St. Louis, MO & F18 & Raleigh, NC \\
F4 & Boise, ID & F9 & Austin, TX & F14 & Chicago, IL & F19 & New Jersey, NY \\
F5 & Phoenix, AZ & F10 & Oklahoma City, OK & F15 & Jackson, MS & F20 & Boston, MA \\
\hline
\end{tabular}

All the parameters in the model are the same as in Section 3.4.1 except the capacity. It is assumed that the capacity of the facilities $\left(c_{l}\right.$, excluding the dummy facility) are all $c_{l}=1,207$. The resulting correlation matrix is shown in Table 3.6. 
Table 3.6: Correlation matrix

\begin{tabular}{|c|c|c|c|c|c|c|c|c|c|c|c|c|c|c|c|c|c|c|c|c|}
\hline & F1 & $\mathrm{F} 2$ & F3 & $\mathrm{F} 4$ & F5 & $\mathrm{F} 6$ & F7 & F8 & F9 & F10 & F11 & F12 & $\mathrm{F} 13$ & F14 & F15 & F16 & F17 & F18 & F19 & $\mathrm{F} 20$ \\
\hline F1 & 1 & 0.917 & 0.5 & 0.614 & 0.390 & 0.274 & 0.290 & 0.239 & 0 & 0.009 & 0.027 & 0.031 & 0 & 0 & 0 & 0 & 0 & 0 & 0 & 0 \\
\hline F2 & 0.917 & 1 & 4509 & 596 & 0.35 & 277 & 0.293 & 261 & 0 & 009 & 0.037 & 0.042 & 0 & 0 & 0 & 0 & 0 & 0 & 0 & 0 \\
\hline F3 & 0.5 & .451 & 1 & 0.565 & 0.74 & 370 & 0.374 & 148 & .127 & 115 & 0.035 & 0 & 0 & 0 & .009 & 0 & 0 & & & 0 \\
\hline $\mathrm{F} 4$ & 0.614 & 596 & 56 & 1 & 0.552 & 505 & 0.527 & 410 & 82 & 147 & 0.154 & 0.140 & .058 & .07 & 0 & c & ( & & & \\
\hline F5 & 90 & 25 & & .55 & 1 & 0.478 & 0.483 & 205 & 15 & 66 & 16 & & & & .056 & 0 & 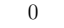 & 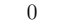 & & \\
\hline F6 & 74 & 277 & & 5 & 478 & 1 & 0.921 & 2 & & 9 & & 18 & 9 & , & 55 & 134 & . 131 & .051 & 0 & 0 \\
\hline F7 & 90 & 0 & & & & 92 & 1 & 0.605 & & & & & & & & & & & & 0 \\
\hline F8 & .239 & 0.261 & & & 0. & 0.562 & 5 & 1 & & & & & & & & & & & & 0 \\
\hline F9 & r & 0 & & & & & & & 1 & 23 & & 6 & & & & & & & & 0 \\
\hline 10 & 009 & 009 & & & 206 & 9 & J & & & 1 & 0.621 & 4 & & & & & & Y & & 04 \\
\hline F11 & 27 & 37 & . & 4 & 0.116 & 4 & 0 . & 9 & 66 & 1 & 1 & 0.765 & 0.694 & 0.71 & 0.5 & & .4 & 0.36 & 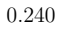 & .14 \\
\hline F12 & 031 & 0.042 & 0 & 0 & 0.057 & 0.348 & 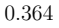 & 99 & & $x$ & 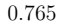 & 1 & 0.611 & 2 & 0.38 & & .4 & .35 & 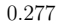 & 2 \\
\hline F1: & 0 & 0 & 0 & 058 & 0.055 & 299 & 0.281 & 259 & 56 & 77 & 0.694 & 0.611 & 1 & 0.859 & 0.667 & 0.7. & $0.7 \mathrm{C}$ & 0.576 & 402 & .293 \\
\hline $\mathrm{F} 1$ & 0 & 0 & 0 & .077 & 0 . & 280 & 0.292 & 295 & 82 & 36 & 0.713 & 0.682 & 0.859 & 1 & 0.546 & 0.6 & 0.72 & 0.591 & .459 & 0.341 \\
\hline F15 & 0 & 0 & .009 & 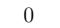 & 0.056 & 0.205 & 0.178 & 111 & 68 & 554 & 0.510 & 0.389 & 0.667 & 0.546 & 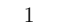 & 0.727 & .581 & 0.55 & 0.35 & 0.225 \\
\hline F16 & 0 & 0 & 0 & 0 & 0 & 134 & 0.119 & 0.092 & 0.292 & 06 & 0.469 & 0.424 & 0.711 & 0.656 & 0.727 & - & 0.776 & 0.783 & .529 & 0.386 \\
\hline $\mathrm{F}$ & 0 & 0 & 0 & 0 & 0 & 31 & 0.141 & 0 & 0.216 & 8 & 0.485 & 0.473 & 77 & 7 & 0.581 & 0.7 & & 0.8 & .594 & .449 \\
\hline $\mathrm{F}$ & 0 & 0 & 0 & 0 & 0 & 0.051 & 2 & 048 & 0.165 & 9 & 0.364 & 0.356 & .576 & .591 & 0.55 & 3 & 0.8 & a & 0.690 & 0.517 \\
\hline F19 & 0 & 0 & 0 & 0 & c & 0 & 0.008 & 0.011 & .034 & .136 & 0.240 & 0.277 & 0.402 & 9 & 0.35 & 29 & 94 & 690 & 1 & 0.756 \\
\hline F20 & 0 & 0 & 0 & 0 & 0 & 0 & 0 & 0 & 0 & 0.049 & 0.147 & 0.2 & 0.293 & 0.341 & 0.225 & 0.386 & 0.449 & 0.517 & 0.756 & 1 \\
\hline
\end{tabular}

Different population sizes and iteration limit are considered to evaluate the performance of the proposed solution approach. Table 3.7 summarizes all parameters used to configure the GA.

Table 3.7: GA parameters

\begin{tabular}{ll}
\hline parameters & values \\
\hline population size & $\{20,25,30\}$ \\
crossover rate & 1 \\
mutation rates & $\{0.1,0.2\}$ \\
scenario sub sampling ratio & 0.1 \\
initial temperature & 1 \\
reduction factor & 0.95 \\
number of iterations & $\{50,100\}$ \\
\hline
\end{tabular}

For purposes of illustration, twelve problem instances are solved considering different GA's parameters. A summary of the hybrid GA-DEA results is shown in Figure 3.7 and Figure 3.8. For example, Figure 3.7(a) shows the GA results when the population size is 20 in each iteration and the GA's process is repeated 50 times with a crossover rate equal to 1 , and a mutation rate equal to 0.1 . The first graph shows the average efficiency score of each generation (iteration). The second graph shows the number of local efficient frontier DMUs per generation. Local efficient frontier DMUs are desirable efficient frontier points (chromosomes) which are calculated after reaching the terminal condition in the GA process as below. 
Step 1: a pool of chromosomes is created considering all the chromosomes from the first generation until the last generation. For example, in Figure 3.7(a), the corresponding pool includes $20 \times 50=1000$ chromosomes.

Step 2: all the corresponding $\left[g_{1}\left(x_{i}\right),\left[g_{2}\left(x_{i}\right), g_{3}\left(x_{i}\right)\right]\right]$ are used as a DMU's input and outputs in the DEA model as previous.

Step 3: chromosomes with the highest efficiency scores are selected as local efficient frontier DMUs.

The general trend of the average efficiency scores among all the experiments is increasing. Also, as is shown, by increasing the iteration number, the total number of local efficient frontier DMUs increases. 

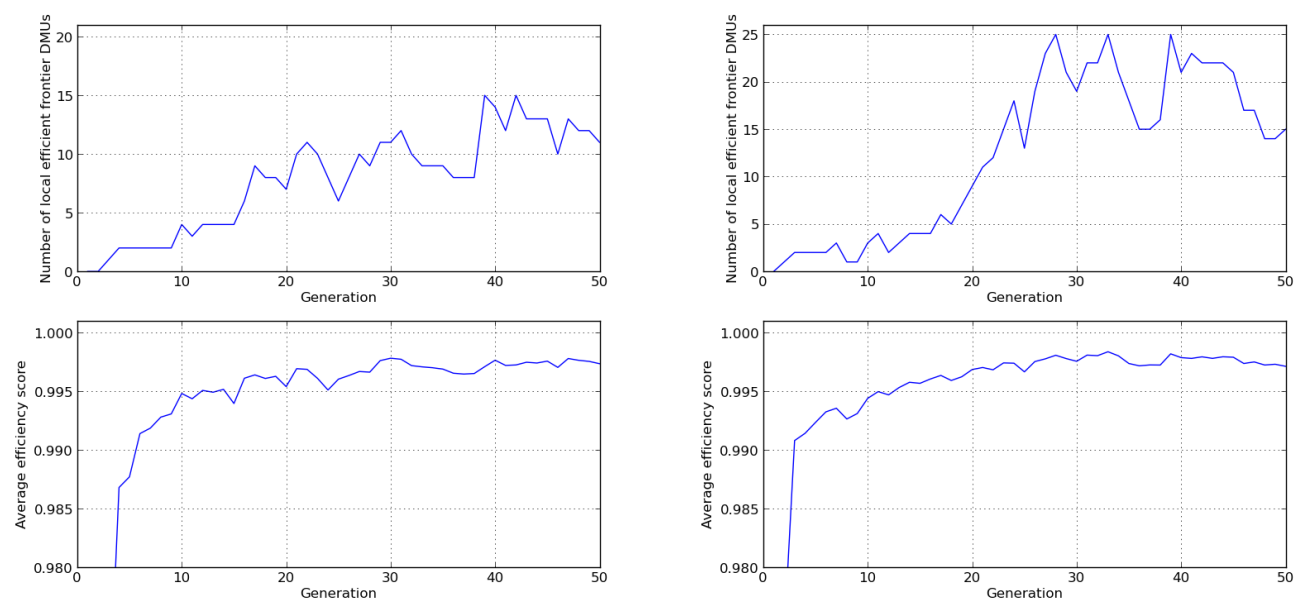

(a) $20,1,0.1$

(b) $25,1,0.1$
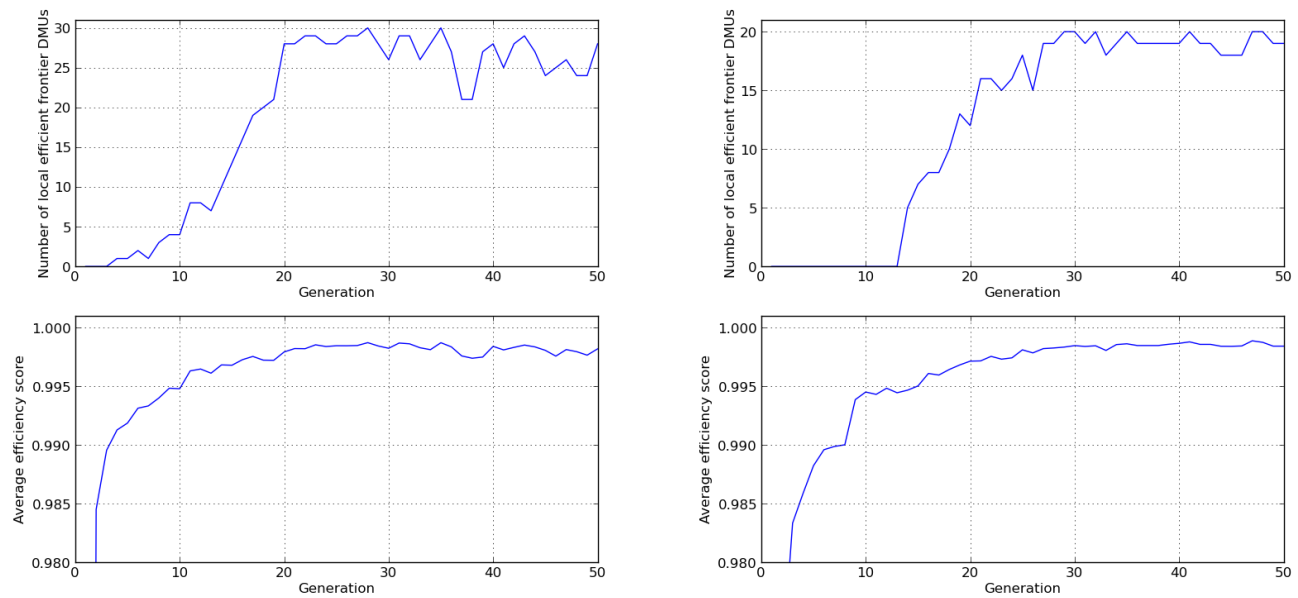

(c) $30,1,0.1$

(d) $20,1,0.2$
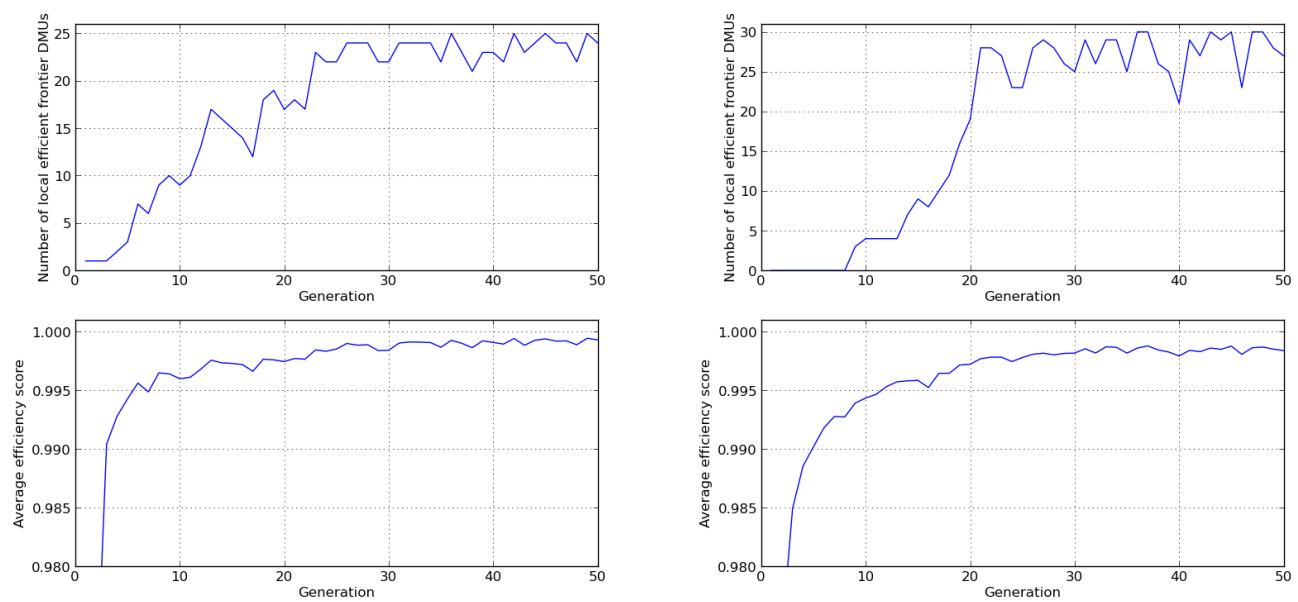

(e) $25,1,0.2$

(f) $30,1,0.2$

Figure 3.7: GA results for a given population number, crossover rate, and mutation rate where required iterations are equal to 50 . 

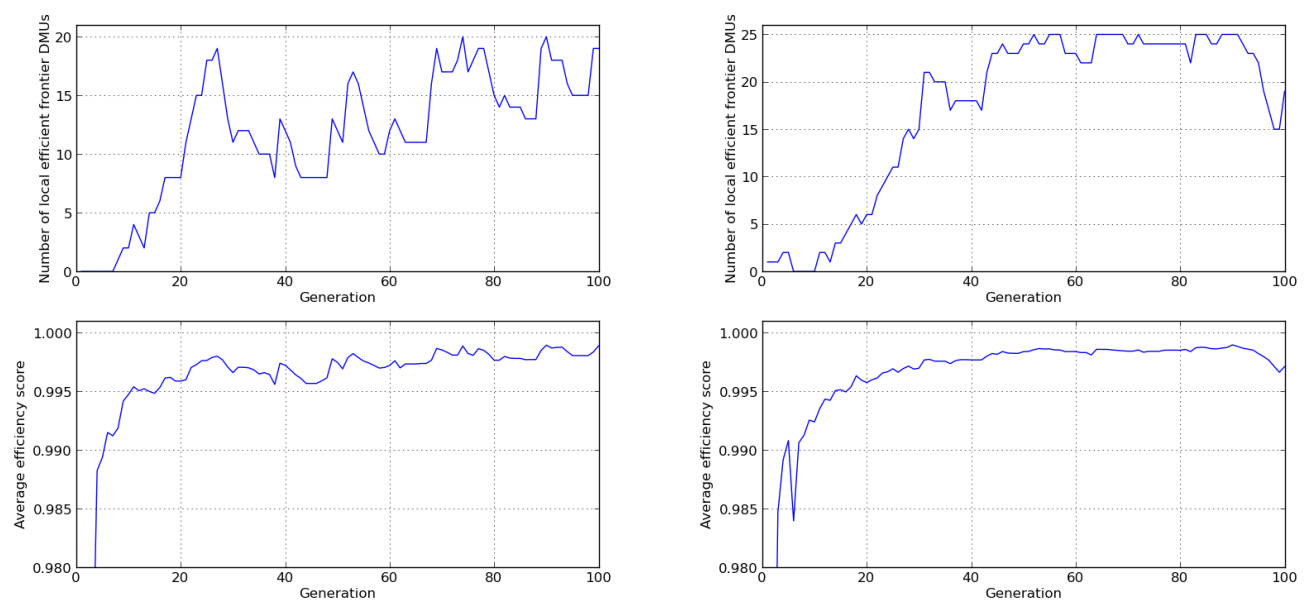

(a) $20,1,0.1$

(b) $25,1,0.1$
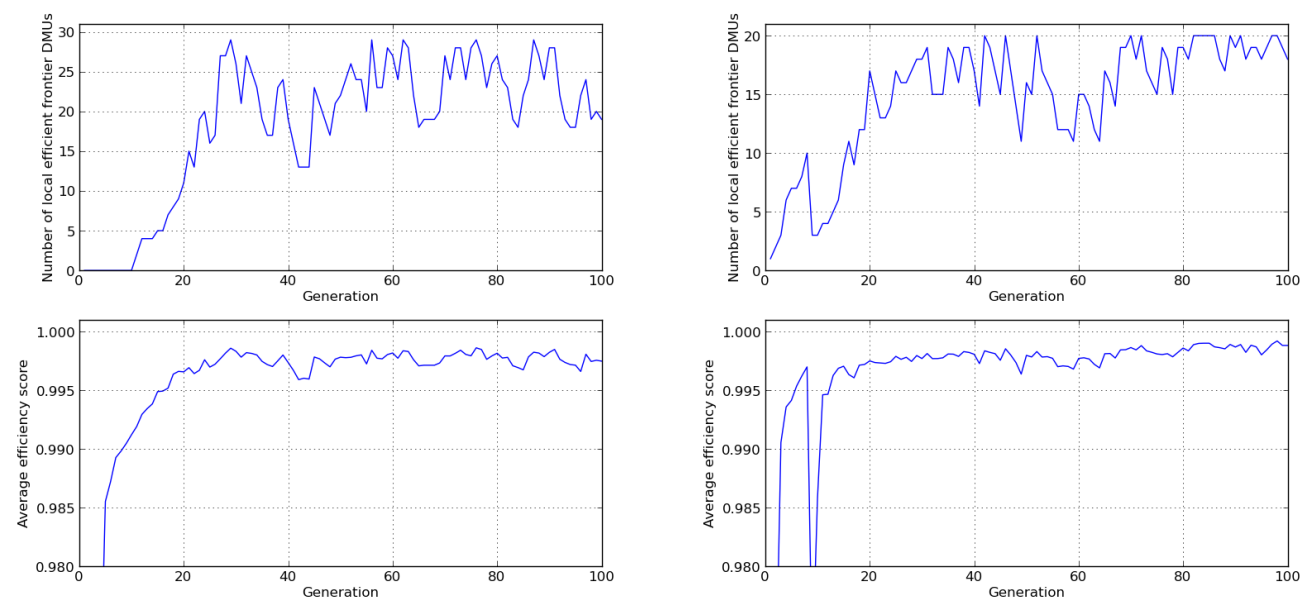

(c) $30,1,0.1$

(d) $20,1,0.2$
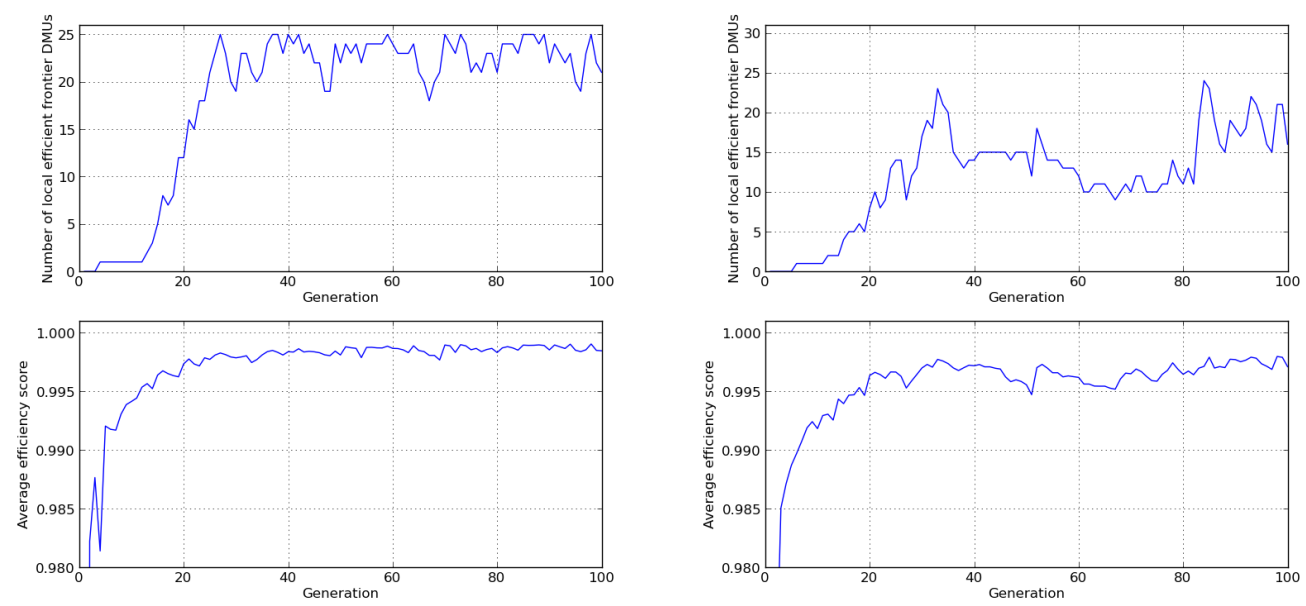

(e) $25,1,0.2$

(f) $30,1,0.2$

Figure 3.8: GA results for a given population number, crossover rate, and mutation rate where required iterations are equal to 100 . 
Analysis of results. As mentioned previously, the supply chain design objective is to identify configurations with the highest potential for consistently meeting a firm's needs at the lowest risk cost. In order to select the best supply chain configuration, it is necessary to make a trade-off between these goals, some of which may be conflicting. The aim of the proposed solution approach is to support decision-makers facing such a problem. Because of the nature of a multi-objective problem, usually no unique optimal solution is found. Therefore, a set of most preferable alternatives are provided. This way, the decision-maker needs to focus only on limited non-dominated solutions for the final choice.

For the purpose of illustration, Table 3.8 shows top five extreme results of the proposed hybrid GA-DEA in terms of $g_{1}, g_{2}, g_{3}, \theta$. For example by looking at top five non-dominated solutions $(\theta=1)$, in two configurations, 10 facilities are used and in the remaining three configurations 9 facilities are used. Opening 10 facilities needs a higher $g_{1}$ (supply chain configuration cost), but leads to less $g_{2}, g_{3}$ (disruption costs). When by opening 9 facilities, the supply chain configuration cost is less but leads to higher disruption costs. Locations of the open facilities (supply chain configuration structure) are another main factor that the decision-maker needs to consider, in addition to cost and risk of supply chain.

All top five worst solutions (lowest $\theta$ ) have 8 open facilities. These configurations lead to saving around $\$ 10,000,000$ in supply chain configuration cost (compared to non-dominated solutions with 9 facilities) but at the same time increase disruption costs by approximately $\$ 25,000,000$.

In the remaining extreme solutions, only supply chain configurations with lowest $g_{2}$ have more or less acceptable solutions, but none of the remaining extreme results lead to reasonable costs. 
Table 3.8: Extreme results

\begin{tabular}{|c|c|c|c|c|c|}
\hline & $x$ & $g_{1}$ & $g_{2}$ & $g_{3}$ & $\theta$ \\
\hline \multirow{5}{*}{ highest $\theta$} & {$[1,0,0,0,1,1,1,1,0,1,1,1,0,0,0,0,0,0,1,1]$} & $101,632,911$ & 0 & 160,987 & 1 \\
\hline & {$[1,1,0,1,0,1,1,1,0,0,0,0,0,1,1,0,0,1,0,1]$} & $101,539,452$ & 0 & 188,025 & 1 \\
\hline & {$[1,0,1,1,0,0,1,1,0,0,1,0,0,0,1,0,1,0,0,1]$} & $91,735,175$ & 579,584 & $3,592,853$ & 1 \\
\hline & {$[1,0,1,0,0,0,1,1,0,1,1,0,0,0,1,1,0,0,0,1]$} & $91,919,252$ & 489,508 & $4,054,018$ & 1 \\
\hline & {$[0,0,1,1,0,0,1,1,0,0,0,0,0,0,1,1,1,0,1,1]$} & $91,867,513$ & 454,028 & $4,530,014$ & 1 \\
\hline \multirow{5}{*}{ lowest $\theta$} & {$[0,0,0,1,0,0,1,0,1,0,0,1,1,0,1,1,1,0,0,0]$} & $81,934,826$ & $19,567,208$ & $6,519,731$ & 0.051522818 \\
\hline & {$[0,0,0,1,0,0,0,0,1,0,0,1,0,0,1,1,1,1,1,0]$} & $82,226,612$ & $19,555,138$ & $6,408,204$ & 0.057992678 \\
\hline & {$[0,0,0,0,0,0,0,1,1,0,1,1,1,0,0,1,0,1,0,1]$} & $82,151,746$ & $19,323,394$ & $6,479,176$ & 0.065143378 \\
\hline & {$[0,0,0,0,0,0,1,0,1,0,0,1,1,0,1,1,1,1,0,0]$} & $82,112,496$ & $19,243,732$ & $6,715,748$ & 0.067784557 \\
\hline & {$[0,0,0,1,0,0,1,0,1,0,1,1,0,0,1,1,0,0,0,1]$} & $82,105,073$ & $19,494,788$ & $6,355,579$ & 0.068016575 \\
\hline \multirow{5}{*}{ highest $g_{1}$} & {$[1,1,1,1,1,1,1,1,1,1,1,1,1,1,1,1,1,1,1,1]$} & $201,221,352$ & 0 & 0 & 0.517485102 \\
\hline & {$[1,1,1,0,1,1,1,1,1,1,1,0,1,0,1,1,1,1,1,1]$} & $171,487,311$ & 0 & 6,040 & 0.606665315 \\
\hline & {$[1,0,1,1,1,1,1,1,1,1,1,0,1,1,1,0,1,1,1,1]$} & $171,372,952$ & 0 & 5,509 & 0.607118199 \\
\hline & {$[1,1,1,1,1,1,1,1,1,1,1,1,1,0,0,1,1,1,1,1]$} & $171,332,100$ & 0 & 5,557 & 0.60725859 \\
\hline & {$[1,1,1,0,1,1,1,1,1,1,1,1,1,0,1,1,1,1,0,1]$} & $171,316,517$ & 0 & 14,960 & 0.606462786 \\
\hline \multirow{5}{*}{ lowest $g_{1}$} & {$[0,1,1,1,1,0,0,0,0,0,1,1,0,1,1,0,0,0,0,0]$} & $81,579,581$ & $19,897,926$ & $4,867,107$ & 0.351357194 \\
\hline & {$[0,0,1,1,1,0,0,1,0,0,0,1,0,0,1,1,1,0,0,0]$} & $81,584,088$ & $19,308,910$ & $5,033,190$ & 0.319773372 \\
\hline & {$[0,1,0,1,1,0,0,1,0,0,0,1,0,0,1,1,0,0,0,1]$} & $81,584,364$ & $19,229,248$ & $5,131,681$ & 0.301053924 \\
\hline & {$[0,0,1,1,0,0,1,1,0,0,0,1,0,0,1,1,1,0,0,0]$} & $81,584,558$ & $19,045,784$ & $5,218,585$ & 0.284537041 \\
\hline & {$[0,1,0,1,0,0,1,1,0,0,0,1,0,0,1,1,0,0,0,1]$} & $81,584,834$ & $18,966,122$ & $5,317,076$ & 0.265817819 \\
\hline \multirow{5}{*}{ highest $g_{2}$} & {$[0,0,0,1,0,1,1,0,1,1,1,1,0,1,0,0,0,0,0,0]$} & $81,790,771$ & $20,455,560$ & $5,443,570$ & 0.241168942 \\
\hline & {$[0,0,0,1,1,1,0,0,0,1,0,1,0,1,1,0,0,1,0,0]$} & $81,700,321$ & $20,441,076$ & $5,147,131$ & 0.297694592 \\
\hline & {$[0,0,0,1,1,1,0,0,0,1,0,1,0,0,0,0,1,1,1,0]$} & $82,727,684$ & $20,320,376$ & $4,565,840$ & 0.402945908 \\
\hline & {$[0,1,0,1,0,1,0,0,1,0,1,1,1,0,0,1,0,0,0,0]$} & $82,291,766$ & $20,320,376$ & $5,903,196$ & 0.153099223 \\
\hline & {$[1,0,0,1,0,1,0,0,0,1,1,0,1,0,0,0,0,1,1,0]$} & $82,924,183$ & $20,310,720$ & $4,854,071$ & 0.348097398 \\
\hline \multirow{5}{*}{ lowest $g_{2}$} & {$[0,1,1,1,0,0,0,1,1,1,0,1,0,0,1,0,0,1,1,0]$} & $101,445,962$ & 0 & 768,730 & 0.988606285 \\
\hline & {$[0,1,1,1,1,0,0,1,0,0,1,1,0,0,1,0,0,1,1,0]$} & $101,447,845$ & 0 & 743,442 & 0.989124225 \\
\hline & {$[0,1,1,1,1,0,1,1,0,0,0,1,0,0,1,0,0,1,1,0]$} & $101,448,775$ & 0 & 599,123 & 0.992175705 \\
\hline & {$[0,1,1,1,1,0,1,1,0,0,0,1,0,0,1,0,1,1,0,0]$} & $101,449,441$ & 0 & 576,511 & 0.992648727 \\
\hline & {$[0,1,1,1,1,0,0,1,0,1,0,1,0,0,1,0,0,1,1,0]$} & $101,450,699$ & 0 & 604,017 & 0.992053112 \\
\hline \multirow{5}{*}{ highest $g_{3}$} & {$[0,0,0,0,0,0,1,0,1,0,0,1,1,0,1,1,1,1,0,0]$} & $82,112,496$ & $19,243,732$ & $6,715,748$ & 0.067784557 \\
\hline & {$[0,0,0,0,0,0,1,0,1,0,0,0,1,0,1,1,1,1,0,1]$} & $82,427,779$ & $18,637,818$ & $6,532,767$ & 0.101287898 \\
\hline & {$[0,0,0,1,0,0,1,0,1,0,0,1,1,0,1,1,1,0,0,0]$} & $81,934,826$ & $19,567,208$ & $6,519,731$ & 0.051522818 \\
\hline & {$[0,0,0,0,0,0,0,1,1,0,1,1,1,0,0,1,0,1,0,1]$} & $82,151,746$ & $19,323,394$ & $6,479,176$ & 0.065143378 \\
\hline & {$[0,0,0,1,0,0,0,0,1,0,0,1,0,0,1,1,1,1,1,0]$} & $82,226,612$ & $19,555,138$ & $6,408,204$ & 0.057992678 \\
\hline \multirow{5}{*}{ lowest $g_{3}$} & {$[1,1,1,1,1,1,1,1,1,1,1,1,1,1,1,1,1,1,1,1]$} & $201,221,352$ & 0 & 0 & 0.517485102 \\
\hline & {$[1,1,1,1,1,1,1,1,1,1,1,1,1,0,1,1,1,0,0,1]$} & $171,290,547$ & 0 & 1,258 & 0.607795078 \\
\hline & {$[1,1,1,1,1,1,1,1,1,1,0,0,1,1,1,1,0,1,0,1]$} & $161,382,861$ & 0 & 1,258 & 0.645109094 \\
\hline & {$[1,1,1,1,1,1,1,1,1,0,1,0,0,1,1,0,0,1,1,1]$} & $151,341,032$ & 0 & 4,251 & 0.687606905 \\
\hline & {$[1,0,1,1,1,1,1,1,1,1,1,0,1,1,1,0,1,1,1,1]$} & $171,372,952$ & 0 & 5,509 & 0.607118199 \\
\hline
\end{tabular}

Reliable vs. unreliable solution. Table 3.9 and Figure 3.9 show the results of two different supply chain configurations in terms of $g_{1}, g_{2}$, and $g_{3}$. As it is shown in Table 3.9, the first configuration is a reliable design (i.e., a robust design which is a non-dominated solution with $\theta=1$ ) while the second configuration is an unreliable design (i.e., dominated solution with $\theta<<1$ ).

Table 3.9: Reliable vs. unreliable solution

\begin{tabular}{|c|ccc|c|}
\hline$x$ & $g_{1}$ & $g_{2}$ & $g_{3}$ & $\theta$ \\
\hline$[1,0,1,1,0,0,1,1,0,0,1,0,0,0,1,0,1,0,0,1]$ & $91,735,175$ & 579,584 & $3,592,853$ & 1 \\
\hline$[0,1,0,1,0,0,1,1,0,0,0,1,0,0,1,1,0,0,0,1]$ & $81,584,834$ & $18,966,122$ & $5,317,076$ & 0.265817819 \\
\hline
\end{tabular}


In the reliable solution, nine facilities are used and in the unreliable solution eight facilities are used. Unreliable solution has $11 \%$ lower $g_{1}$ (supply chain configuration cost) than reliable solution. However, unreliable solution leads to much higher $g_{2}$ and $g_{3}$ (disruption costs). In other words, reliable solution results in $3100 \%$ saving on $g_{2}$ and $48 \%$ saving on $g_{3}$. In addition, in this particular example, the reliable solution leads to $10 \%$ saving on the summation of costs (i.e, $\left.g_{1}+g_{2}+g_{3}\right)$ compared to unreliable solution.

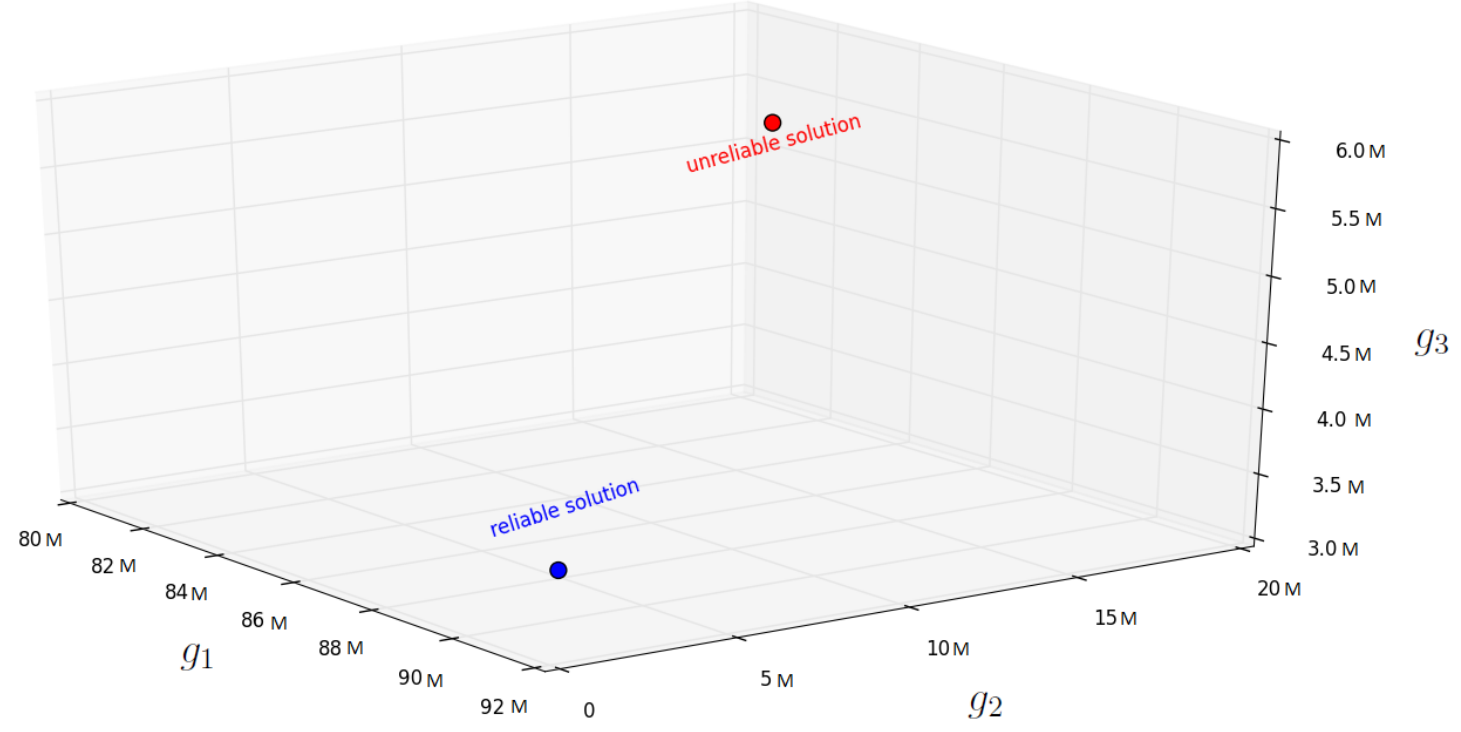

Figure 3.9: Reliable vs. unreliable solution

\subsection{Conclusions}

In this dissertation, a specific focus on the risks inherent in the supply chain was given to understand the impact of different disruptions on the cost of the supply chain. A multi-objective optimization model and solution algorithm was proposed to explore the tradeoffs between costs and risk in the supply chain. Two types of disruption (disruption in quality and/or quantity of the products), were considered and stochastic formulations were proposed attempt to capture uncertainty in the model. An efficient hybrid metaheuristic approach was proposed given some difficulties in the 
current large-scale stochastic optimization solutions. The multiple Pareto-optimal solutions were generated by using a Genetic Algorithm, in which Data Envelopment Analysis was used to calculate the fitness value of supply chain configurations. In addition, several modifications to the basic genetic algorithm were applied. For example, scenario sub-sampling was considered to evaluate offspring. Also simulated annealing approach is used to re-evaluate the acceptance of candidate chromosome. The numerical experiments indicate that these adjustment help to accelerate the GA search process and also extend the visited areas of feasible region. The findings from this dissertation can help practitioners while designing supply chains, as well as help policymakers understand the impact of different disruption mitigation strategies on cost and risk in the supply chain. 


\title{
CHAPTER 4. A DYNAMIC PROGRAMMING APPROACH FOR OPTIMAL INSPECTION SCHEDULING
}

\begin{abstract}
Inspection of suppliers is critical to ensure that only high-quality products reach the marketplace. The Food and Drug Administration (FDA) requires inspection of pharmaceutical suppliers every two years [44]. This may not be the most effective or efficient policy because of the limited number of inspection personnel, and because of differences in supplier characteristics. To this end, a dynamic programming (DP) model is proposed to determine an optimal inspection schedule for a group of suppliers. This schedule can move from an in-compliance state to an out-of-compliance state according to a known probability distribution. A supplier can be in an out-ofcompliance state due to operator error, machine error, defective raw materials, etc. Numerical experiments indicate that the proposed DP model results in significantly lower costs than the FDA's current inspection policy.
\end{abstract}

\subsection{Introduction and motivation}

In quality management, inspection is an important method for controlling and raising the level of product quality. The quality of a system can improve by considering inspection of the units received from suppliers. Especially in some cases, such as pharmaceutical suppliers, inspection is critical in ensuring that only high-quality drugs reach patients. Distributing a tainted drug would obviously have a significant impact on patients' health, even leading to death. This has actually occurred, such as the Heparin case. Heparin is a widely used anticoagulant and produced from the mucosal tissues of pigs. In 2008, at least 12 Chinese companies produced and shipped tainted Heparin throughout the world. Contaminated Heparin caused 81 deaths and several 
allergic reactions in the United States and around the world. Therefore, further testing and recalls of Heparin have occurred by the U.S. Food and Drug Administration [5]. Distribution of the contaminated Heparin has had a serious impact on the health of patients. However, there is always a high chance of a Heparin shortage which may endanger the health and safety of patients. As this example clearly shows, inspection can be an effective method to ensure that only high-quality products reach to customers when a disruption occurs.

There are several other similar cases in the food industry. For example, 1,500 people were reportedly sickened by consuming contaminated eggs [10]. Similarly in Germany in 2011, lettuce, cucumbers, and tomatoes were recalled as a result of the deaths of 31 people and the grave illness of more than 3,000 [9].

These examples indicate the danger of failures in various industries and also underscore the need for policies and interventions that help with quicker detection of quality problems and resultant corrective actions. In addition, by preventing potential failure, the disruption cost would be cut out. For example, The FDA requires inspection of pharmaceutical suppliers at least once every two years [44]. Further, the Maine Department of Transportation (MDOT) inspects all bridges every 24 months [81].

Motivated by these cases, in this dissertation, the operational inspection decision is examined for a given supply chain with multiple unreliable supply sources. Disruption directly affects the quality of products. The quality of products can be improved by considering inspection of the units received from the suppliers. Even for a robust supply chain, it is essential to optimize inspection decisions to ensure quality. A dynamic programming model is developed to indicate which set of suppliers need inspection at various periods, according to the various cost terms. In addition, an efficient approximate dynamic programming algorithm is developed to solve the model. The current FDA inspection policy is evaluated; numerical experiments in- 
dicate that proposed DP models result in significantly lower costs than the FDA's current inspection policy.

\subsubsection{Literature review}

There is a vast literature $[$ e.g., $82,83,14,84,85,86,87,88,15,89,16,90]$ on inspection in the context of production systems and supply chains. For example, Hariga and Azaiez [15] discuss about inspection schedules mechanism for an unreliable facility where a minimal repair is carried out. A non-Markovian failure process with increasing failure rate is used to formulate the problem and three cost-effective heuristic procedures are developed. Anily and Grosfeld-Nir [89] study optimal policy for a lot-sizing (batch production) and off-line inspection problem to minimize the expected total of production, inspection, and shortage costs. A finite-time partially observable Markov decision process is used to formulate the inspection problem where the failure time is geometrically distributed. They show that the optimal policy for the inspection problem has a control limit threshold structure. Chun [16] designs a Bayesian inspection procedure for the production process which is subject to random failure. The model simultaneously answers three main questions: how often to inspect; how to search; and when to stop the search. A stochastic dynamic programming approach is used to formulate the renewal-reward process as a profit-maximization model considering several cost factors. Also several ways of estimating are provided for the failure rate by Bayesian methods. Wang et al. [90] investigate an off-line inspection policy for a production batch produced from an unreliable process under a required confidence level. An approach based on an information theory of entropy is developed which selects the un-inspected unit to minimize the uncertainty of the transition unit.

A related branch of literature studies machine replacement and maintenance optimization problem. For example, McClurg and Chand [91] study parallel machine replacement problem and present a forward-time dynamic programming algorithm to 
minimizes the sum of the discounted costs for a finite horizon problem. The number of possible options is reduced by using dominance property. A forecast horizon result is presented. Childress and Durango-Cohen [92] study stochastic parallel machine replacement problem as a set of $N$ independent Markovian processes. General replacement-cost function is used and the structures of optimal policies (No-Splitting and Worse Cluster Replacement Rules) are used to reduce the computational effort to solve the problem. Guillaumot et al. [93] study joint inspection and maintenance policies for infrastructure facilities. An adaptive optimization model is performed by combining the latent MDP with the adaptive control formulations to minimize the total expected social cost of managing facilities over a finite planning horizon. The case study result shows that providing the wrong information may be less costly than providing no information about deterioration. Also, when the initial beliefs are adequate, consideration of a flexible inspection schedule leads to considerable benefits.

Additional related research has been completed by Rosenkrantz et al. [57] who study the placement of inspection stations on networks (such as transportation and communication). They use two different optimization objectives. In the first optimization problem, the objective minimizes the maximum interval between two successive inspection stations along the path. In the second category, the objective minimizes the total cost, which includes inspection cost and expected penalty cost. Polynomial time algorithms and fully polynomial approximations are presented for analyzing the results. Ozekici and Pliska [94] develop a dynamic inspection strategy to choose an inspection schedule which minimizes the expected value of the costs. Their model adjusts for medical screening and considers four kinds of costs: inspection costs; false positive costs (e.g., cost of doing the super test); true positive costs (when a defect is detected); and finally failure cost (whether resulting from unsuccessful treatment or not). The model is applied to two sample problems in medicine: post-operative periumbilical pruritis; and breast cancer. Also, for a research overview 
in inspection allocation problem, the reader is refereed to study Shetwan et al. [95].

In this dissertation, a supply chain system with multiple (i.e., two or more) unreliable supply sources is studied that have different grades of reliability, in terms of the quality of orders delivered. A supplier can be in an out-of-compliance state due to several reasons that can include operator errors, machine errors, defective raw materials, etc. It is assumed that suppliers can move from an in-compliance state to an out-of-compliance state according to a known probability distribution. Considering capacity constraint and a constrained Markov decision program (MDP), a dynamic programming model is developed to find an optimal inspection schedule for suppliers. The suppliers' last known condition and the number of periods since the last inspection are used in the model for identifying a group of the suppliers that must be inspected. Cost function includes the cost of inspection, cost of corrective actions, and the cost of missed detection of a low-quality supply from suppliers. The structural properties of the proposed DP model are then examined for a single supplier. The properties of the single supplier are then incorporated to multi suppliers problem to develop an effective approximate DP algorithm to find high-quality solutions. The numerical experiments indicate that the proposed DP model can result in significantly lower costs than the FDA's current inspection policy.

The remainder of this chapter is organized as follows. In Section 4.2, the problem description is presented and formulated as a DP model. In Section 4.3, the structural property of proposed DP is discussed in a single supplier version, including threshold policy. In Section 4.4, linear programming approach is discussed as an alternative model. An effective approximate dynamic programming algorithm is introduced in Section 4.5. Computational results are presented in Section 4.6. Finally Section 4.7 summarizes the findings. 


\subsection{Problem description and notation}

Consider a supply chain system with multiple unreliable supply sources that are subject to random failures at each time period (see Figure 4.1). If a supplier fails to produce high-quality products, it's status can be revealed (and therefore corrected) via an inspection process. Therefore, at the beginning of each time period, a decision as to whether or not to inspect a supplier or not must be made. It is assumed that the inspection decisions are made and the results are revealed at the beginning of the period. In a supply chain with $K$ suppliers, if the supplier $i$ is known to be "in compliance (IC)" at the beginning of time period $t$, it is assumed that the materials received from supplier $i$ in period $t$ are not tainted. However, it can transition into "out-of-compliance (OC)" status with a positive probability at the beginning of period $t+1$ and therefore supply low-quality material in period $t+1$ and will continue supplying the low-quality material until it is inspected.

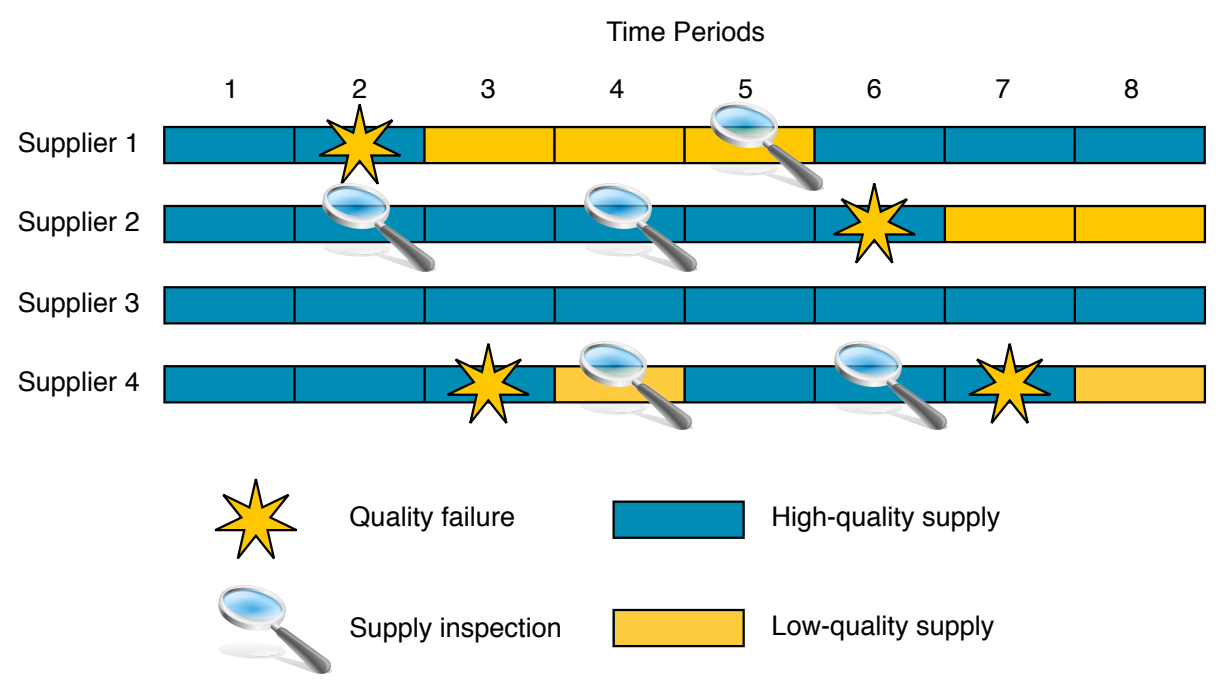

Figure 4.1: Supply Inspection

Without any inspection, a supplier that is OC will keep supplying low quality product indefinitely. On the other hand, a supplier that is IC might transition to OC status with probability $\theta_{i}$ or stay in IC status with probability $\left(1-\theta_{i}\right)$. The underlying 
Markov process of this system without the effect of decisions (i.e., inspection decision) is represented in Figure 4.2.

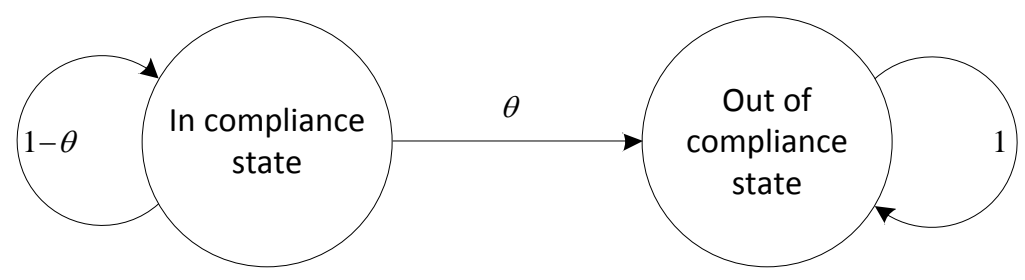

Figure 4.2: Markov process for a given supplier without inspection

The Markov process explained above is for a system without any inspection decisions. Based on the results of inspection process, however, the suppliers can be forced to complete certain corrective actions and therefore the quality of the supplied material would change. Specifically, in this model it is assumed that, if the result of inspection on supplier $i$ at the beginning of period $t$ revealed the supplier to be $\mathrm{OC}$, then the corrective actions will be performed and the material supplied in period $t+1$ will be high quality with probability one. The material supplied in period $t$, however, will be low quality and therefore it will incurs a shortage cost in period $t$. On the other hand, if the supplier is found to be IC, then no corrective actions are taken and the supplier might become OC or stay IC with probabilities $\theta_{i}$ and $\left(1-\theta_{i}\right)$, respectively. A schematic representation of the model with the inspection decisions and the corresponding costs are provided in Figure 4.3. 


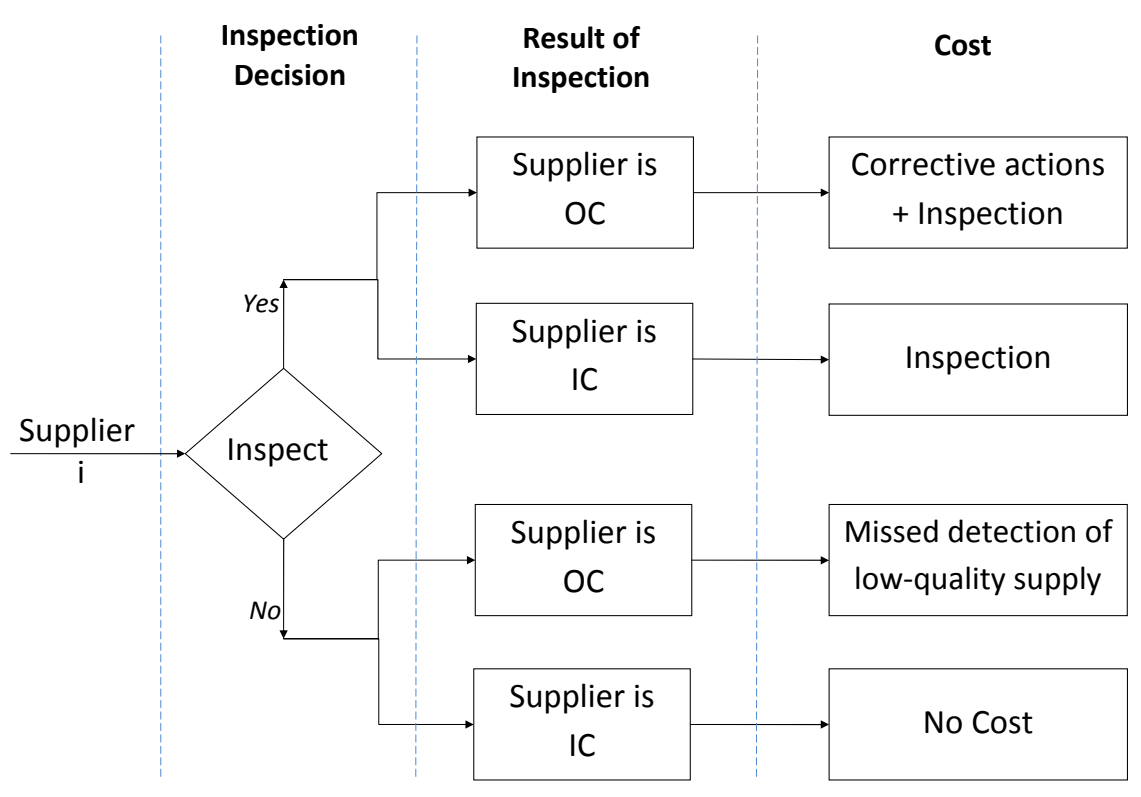

Figure 4.3: Decision tree (IC: in compliance, OC: out of compliance)

Sets and Parameters. Suppose there are products from $k$ sources, indexed by $i=1, \ldots, k$ while they are independent of each other.

- Infinite horizon Markov decision process model

- $k$ is the total number of suppliers.

- $n$ is the maximum number of inspections in a period (i.e., capacity limit).

- $\theta_{i}$ : probability that state moves from in-compliance to another in one period for supplier $i$.

- $s$ is the inspection cost of a supplier if capacity is not exceeded (assume that the inspection cost is same for all suppliers).

- $\bar{s}$ is the inspection cost of each supplier that has to be inspected beyond capacity limit (assume that the inspection cost is same for all suppliers). 
- $r_{i}$ is the cost of corrective actions to move supplier $i$ from an out-of-compliance to an in-compliance state (i.e., discard cost, cost of unsatisfied demand, and cost of legal actions).

- $h_{i}$ : cost of shipped tainted material to customers from supplier $i$ (i.e., cost of missed detection of low-quality supply).

- $\gamma$ : is the one-period discount factor (as the present value of one dollar received one period from now)

\subsubsection{Dynamic Programming Formulation}

Assume there is a limit on the number of periods that a supplier can operate without inspection. i.e., need to inspect each supplier at least once every $\rho+1$ periods. To ensure this, the capacity constraint can be relaxed but assigned a high cost if the "capacity" is exceeded.

State. In the proposed model, a state is represented by the tuple of two vectors.

$\mathbf{z}=(\mathbf{x}, \mathbf{y})=\left(\left[\begin{array}{c}x_{1}^{t} \\ x_{2}^{t} \\ \vdots \\ x_{k}^{t}\end{array}\right],\left[\begin{array}{c}y_{1}^{t} \\ y_{2}^{t} \\ \vdots \\ y_{k}^{t}\end{array}\right]\right) \mathbf{z} \in \mathbf{Z}=\left\{(\mathbf{x}, \mathbf{y}) \mid x_{i}^{t} \leq \rho+1, x_{i}^{t} \in \mathbb{N}, y_{i}^{t} \in\{0,1\} \forall i\right\}$

where

- $x_{i}^{t}$ is the number of periods past since the last inspection of supplier $i$ at time period $t$,

- $y_{i}^{t}$ is the last known condition of supplier $i$.

$y_{i}^{t}= \begin{cases}1, & \text { if the last inspection showed in compliance state at time period } t \\ 0, & \text { if the last inspection showed out of compliance state at time period } t\end{cases}$ 
Action. The action space is either to inspect the supplier or to do nothing. $a_{i}^{t}$ is the decision regarding the inspection of supplier $i$

$$
a_{i}^{t}= \begin{cases}1, & \text { if supplier } i \text { is inspected at time period } t \\ 0, & \text { otherwise. }\end{cases}
$$

Let $\mathcal{A}(\mathbf{z})$ be the set of feasible actions given state $\mathbf{z}$ defined as follows:

$$
\mathcal{A}(\mathbf{z})=\left\{\left[\begin{array}{c}
a_{1}^{t} \\
a_{2}^{t} \\
\vdots \\
a_{k}^{t}
\end{array}\right] \mid a_{i}^{t} \in\{0,1\} \text { and } a_{i}^{t}=1 \text { if } x_{i}^{t} \geqslant \rho+1\right\}
$$

State Transitions. State transition is defined by:

$$
\mathbf{z}=\left(\left[\begin{array}{c}
x_{1}^{t} \\
x_{2}^{t} \\
\vdots \\
x_{k}^{t}
\end{array}\right],\left[\begin{array}{c}
y_{1}^{t} \\
y_{2}^{t} \\
\vdots \\
y_{k}^{t}
\end{array}\right]\right) \Longrightarrow \mathbf{z}^{\prime}=\left(\left[\begin{array}{c}
x_{1}^{t+1} \\
x_{2}^{t+1} \\
\vdots \\
x_{k}^{t+1}
\end{array}\right],\left[\begin{array}{c}
y_{1}^{t+1} \\
y_{2}^{t+1} \\
\vdots \\
y_{k}^{t+1}
\end{array}\right]\right)
$$

where

$$
\begin{gathered}
x_{i}^{t+1}=\left\{\begin{array}{lll}
x_{i}^{t}+1, & \text { if } & a_{i}^{t}=0, \\
1, & \text { if } & a_{i}^{t}=1 .
\end{array}\right. \\
y_{i}^{t+1}=\left\{\begin{array}{lll}
y_{i}^{t}, & \text { if } & a_{i}^{t}=0, \\
\zeta_{i}^{t}, & \text { if } & a_{i}^{t}=1 .
\end{array}\right.
\end{gathered}
$$

where $\zeta_{i}^{t}$ is the current actual condition of supplier that would be revealed if the supplier is inspected. It is important to emphasize the distinction between $\zeta_{i}^{t}$ and 
$y_{i}^{t}$; the former represents the actual state of the supplier at the beginning of period $t$, whereas the latter denotes the last known state of the supplier which might be different from the actual state. Given the current state at time $t$, the probability distribution of $\zeta_{i}^{t}$ can be calculated as follows:

$$
\begin{aligned}
& \operatorname{Prob}\left\{\zeta_{i}^{t}=0 \mid x_{i}^{t}=1, y_{i}^{t}=1\right\}=\theta_{i} \\
& \operatorname{Prob}\left\{\zeta_{i}^{t}=1 \mid x_{i}^{t}=1, y_{i}^{t}=1\right\}=1-\theta_{i} \\
& \operatorname{Prob}\left\{\zeta_{i}^{t}=0 \mid x_{i}^{t}=1, y_{i}^{t}=0\right\}=0 \\
& \operatorname{Prob}\left\{\zeta_{i}^{t}=1 \mid x_{i}^{t}=1, y_{i}^{t}=0\right\}=1
\end{aligned}
$$

Above, $x_{i}^{t}=1, y_{i}^{t}=1$ means the supplier $i$ was inspected at the beginning of period $t-1$ and it was IC according to the result of the inspection. Therefore in period $t$, the supplier $i$ might have transitioned to an OC state with a probability $\theta_{i}$. On the other hand, the given information $\left(x_{i}^{t}=1, y_{i}^{t}=0\right)$ means the supplier $i$ was inspected at the beginning of period $t-1$ and the result was OC. Therefore, according to the assumptions, corrective actions are taken and the supplier transitioned into IC state with probability 1 in period $t$. Now, given the state at the beginning of period $t$ (i.e., $x_{i}^{t}$ and $y_{i}^{t}$ ) and the decision to inspect or not $\left(i . e ., a_{i}^{t}\right)$, the probability distribution on the state transitions in one period can be calculated as follows:

$$
\begin{aligned}
& \operatorname{Prob}\left\{x_{i}^{t+1}=1, y_{i}^{t+1}=0 \mid x_{i}^{t}=1, y_{i}^{t}=1, a_{i}^{t}=1\right\}=\theta_{i} \\
& \operatorname{Prob}\left\{x_{i}^{t+1}=1, y_{i}^{t+1}=1 \mid x_{i}^{t}=1, y_{i}^{t}=1, a_{i}^{t}=1\right\}=1-\theta_{i} \\
& \operatorname{Prob}\left\{x_{i}^{t+1}=2, y_{i}^{t+1}=1 \mid x_{i}^{t}=1, y_{i}^{t}=1, a_{i}^{t}=0\right\}=1 \\
& \operatorname{Prob}\left\{x_{i}^{t+1}=1, y_{i}^{t+1}=1 \mid x_{i}^{t}=1, y_{i}^{t}=0, a_{i}^{t}=1\right\}=1 \\
& \operatorname{Prob}\left\{x_{i}^{t+1}=2, y_{i}^{t+1}=0 \mid x_{i}^{t}=1, y_{i}^{t}=0, a_{i}^{t}=0\right\}=1
\end{aligned}
$$


Above, Equations 4.7 and 4.8 represent the probability distribution if the supplier was inspected in period $t-1$ and the result was IC and it is decided to inspect again in period $t$. Equation 4.9 states that if the supplier was inspected in period $t-1$ and the result was IC and it is decided not to inspect, the state in period $t+1$ will be $\left(x_{i}^{t+1}=2, y_{i}^{t+1}=1\right)$. According to Equation 4.10, if the supplier was inspected in period $t-1$ and the result was $\mathrm{OC}$ and if it is decided to inspect, we will find the supplier to be IC with probability one (because of the corrective actions). Finally, if the supplier was inspected in period $t-1$ and the result was OC, even though we would know that the supplier is IC in period $t+1$ we would transition to state $\left(x_{i}^{t+1}=2, y_{i}^{t+1}=0\right)$, in which the last known condition is kept at OC (See Figure 4.4 for a schematic representation of these transition probabilities). If more than one period has passed since the last inspection, the probability distribution on $\zeta_{i}^{t}$ can be calculated as follows:

$$
\begin{aligned}
& \operatorname{Prob}\left\{\zeta_{i}^{t}=0 \mid x_{i}^{t}=m, y_{i}^{t}=1\right\}=1-\left(1-\theta_{i}\right)^{m} \\
& \operatorname{Prob}\left\{\zeta_{i}^{t}=1 \mid x_{i}^{t}=m, y_{i}^{t}=1\right\}=\left(1-\theta_{i}\right)^{m} \\
& \operatorname{Prob}\left\{\zeta_{i}^{t}=0 \mid x_{i}^{t}=m, y_{i}^{t}=0\right\}=1-\left(1-\theta_{i}\right)^{m-1} \\
& \operatorname{Prob}\left\{\zeta_{i}^{t}=1 \mid x_{i}^{t}=m, y_{i}^{t}=0\right\}=\left(1-\theta_{i}\right)^{m-1}
\end{aligned}
$$

The probabilities given in Equations 4.12 and 4.13 are obtained for a supplier that is known to be IC $m$ periods ago using the Markovian process explained above. On the other hand, if the last known condition of the supplier is OC, then it is known that it will be IC in the next period with probability one. Therefore, the same Markov chain transition probabilities for $m-1$ periods can be used as given in Equations 4.14 and 4.15. Using these Equations 4.12-4.15, the state transition probabilities can be 

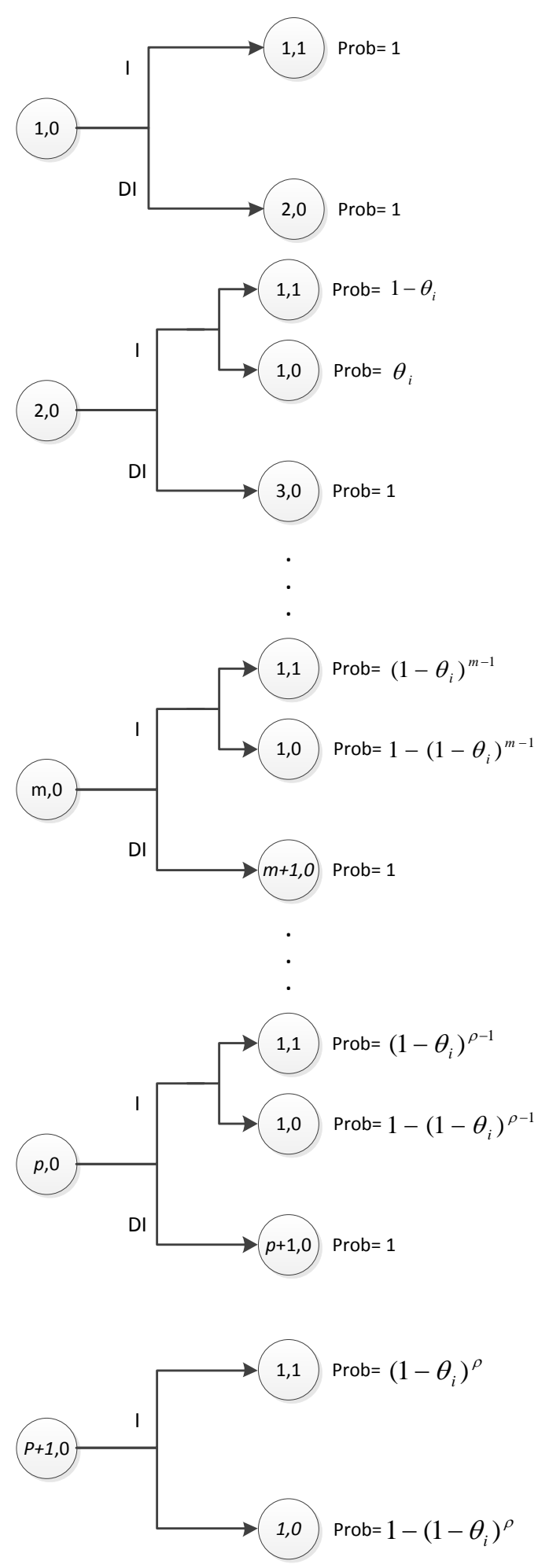
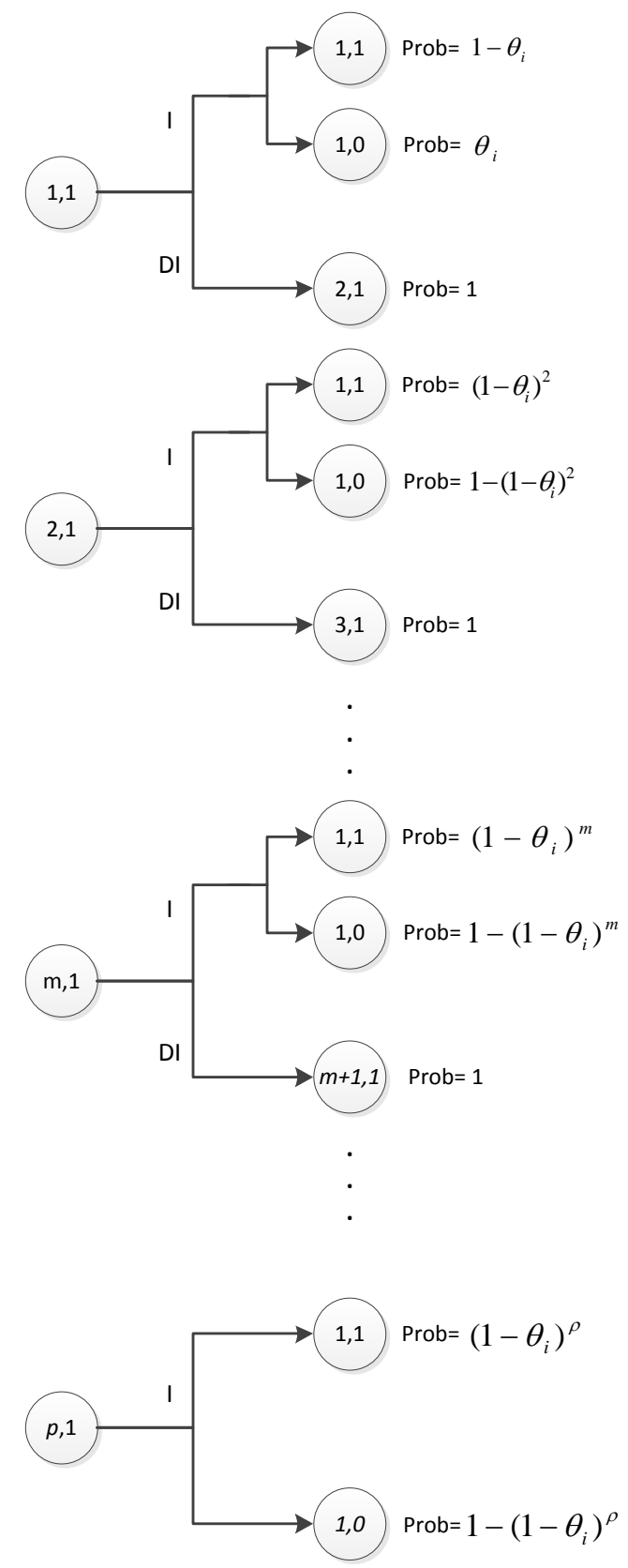

Figure 4.4: State transition diagrams 
generalized as follows:

$$
\begin{aligned}
& \operatorname{Prob}\left\{x_{i}^{t+1}=1, y_{i}^{t+1}=0 \mid x_{i}^{t}=m, y_{i}^{t}=1, a_{i}^{t}=1\right\}=1-\left(1-\theta_{i}\right)^{m} \\
& \operatorname{Prob}\left\{x_{i}^{t+1}=1, y_{i}^{t+1}=1 \mid x_{i}^{t}=m, y_{i}^{t}=1, a_{i}^{t}=1\right\}=\left(1-\theta_{i}\right)^{m} \\
& \operatorname{Prob}\left\{x_{i}^{t+1}=m+1, y_{i}^{t+1}=1 \mid x_{i}^{t}=m, y_{i}^{t}=1, a_{i}^{t}=0\right\}=1 \\
& \operatorname{Prob}\left\{x_{i}^{t+1}=1, y_{i}^{t+1}=0 \mid x_{i}^{t}=m, y_{i}^{t}=0, a_{i}^{t}=1\right\}=1-\left(1-\theta_{i}\right)^{m-1} \\
& \operatorname{Prob}\left\{x_{i}^{t+1}=1, y_{i}^{t+1}=1 \mid x_{i}^{t}=m, y_{i}^{t}=0, a_{i}^{t}=1\right\}=\left(1-\theta_{i}\right)^{m-1} \\
& \operatorname{Prob}\left\{x_{i}^{t+1}=m+1, y_{i}^{t+1}=0 \mid x_{i}^{t}=m, y_{i}^{t}=0, a_{i}^{t}=0\right\}=1
\end{aligned}
$$

Dynamic Programming Recursion. In this section, inspection process in the context of a system with multiple unreliable supply sources is examined, focusing on inter-stage coordination with capacity limits. In other words, there are restrictions in the number of available inspection personnel.

Immediate cost. Let $c(\mathbf{z}, \mathbf{a})$ be a function representing the immediate cost of action a while in state $\mathbf{z}=(\mathbf{x}, \mathbf{y})$. Immediate $\operatorname{cost} c(\mathbf{z}, \mathbf{a})$ includes the cost of inspection, cost of corrective actions, and the cost of missed detection of a low-quality supply from suppliers.

$c(\mathbf{z}, \mathbf{a})=$ Inspection cost + expected corrective action cost + expected missed detection cost

$$
\begin{aligned}
c(\mathbf{z}, \mathbf{a})= & s \cdot \min \left\{\sum_{i=1}^{k} a_{i}, n\right\}+\bar{s} \cdot \max \left\{\sum_{i=1}^{k} a_{i}-n, 0\right\}+ \\
& \sum_{i=1}^{k} a_{i} \cdot r_{i} \cdot \operatorname{Prob}\left\{\zeta_{i}=0 \mid x_{i}, y_{i}\right\}+ \\
& \sum_{i=1}^{k}\left(1-a_{i}\right) \cdot h_{i} \cdot \operatorname{Prob}\left\{\zeta_{i}=0 \mid x_{i}, y_{i}\right\}
\end{aligned}
$$

Note that in this section the time index $t$ is omitted to simplify the notation. Therefore, $\operatorname{Prob}\left\{\zeta_{i}=0 \mid x_{i}, y_{i}\right\}$ denotes the probability that the actual "current" 
state of supplier is OC given the "current" state is $\left(x_{i}, y_{i}\right)$ as defined in Equations 4.12 and 4.14 .

Let $J(\mathbf{z})$ denote the value of being in state $\mathbf{z}$. The Bellman equation can be written as

$$
\begin{aligned}
& J(\mathbf{z})=\infty \quad \text { if } x_{i}^{t}>\rho+1 \quad \text { for any } i \\
& J(\mathbf{z})=\min _{\mathbf{a} \in \mathbf{A}}\left\{c(\mathbf{z}, \mathbf{a})+\sum_{\mathbf{z}^{\prime} \in \Omega(\mathbf{z}, \mathbf{a})} \gamma \cdot \operatorname{Prob}\left\{\mathbf{z}^{\prime} \mid \mathbf{z}, \mathbf{a}\right\} \cdot J\left(\mathbf{z}^{\prime}\right)\right\}
\end{aligned}
$$

where $\Omega(\mathbf{z}, \mathbf{a})$ denotes the set of states that can be transition into given the current state $\mathbf{z}$ and action $\mathbf{a}$ and

$$
\operatorname{Prob}\left\{\mathbf{z}^{\prime} \mid \mathbf{z}, \mathbf{a}\right\}=\prod_{i=1}^{k} \operatorname{Prob}\left\{x_{i}^{\prime}, y_{i}^{\prime} \mid x_{i}, y_{i}, a_{i}\right\}
$$

Note that, since it is assumed that all suppliers are independent, the product of all the transition probabilities for individual suppliers can be used as shown in Equation 4.25 .

\subsection{Single supplier}

In this section, the structural properties of the optimal inspection policies for a single supplier version of the supply chain inspection problem are examined. The results of structural properties for a single supplier version will be used to develop an effective approximate DP algorithm to retain a fairly good solution. Consider the DP model given in Equation 4.24 for only one supplier and ignore the cost $\bar{s}$ of exceeding 
inspection capacity. Then the model simplifies to:

$$
J(x, y)=\min \left\{\begin{array}{c}
s+r \cdot \mathrm{P}\{\zeta=0 \mid x, y\}+\gamma[\mathrm{P}\{x=1, y=0 \mid x, y, a=1\} J(1,0)+ \\
\mathrm{P}\{x=1, y=1 \mid x, y, a=1\} J(1,1)], \\
h \cdot \operatorname{Prob}\{\zeta=0 \mid x, y\}+\gamma \cdot J(x+1, y)
\end{array}\right.
$$

where the first term represents the cost of inspecting and the second term is for not inspecting. More specifically:

$$
\begin{aligned}
& J(x, 0)=\min \left\{\begin{array}{l}
s+r\left(1-(1-\theta)^{x-1}\right)+\gamma\left[\left(1-(1-\theta)^{x-1}\right) J(1,0)+\right. \\
\left.(1-\theta)^{x-1} J(1,1)\right], \\
h\left(1-(1-\theta)^{x-1}\right)+\gamma \cdot J(x+1,0)
\end{array}\right. \\
& J(x, 1)=\min \left\{\begin{array}{l}
s+r\left(1-(1-\theta)^{x}\right)+\gamma\left[\left(1-(1-\theta)^{x}\right) J(1,0)+\right. \\
\left.(1-\theta)^{x} J(1,1)\right], \\
h\left(1-(1-\theta)^{x}\right)+\gamma \cdot J(x+1,1)
\end{array}\right.
\end{aligned}
$$

and the following are obtained when $x=1$

$$
\begin{aligned}
& J(1,0)=\min \{s+\gamma J(1,1), \gamma J(2,0)\} \\
& J(1,1)=\min \{s+r \theta+\gamma[\theta J(1,0)+(1-\theta) J(1,1)], h \theta+\gamma J(2,1)\}
\end{aligned}
$$

When $x=\rho$ :

$$
\begin{array}{r}
J(\rho+1,0)=s+r\left(1-(1-\theta)^{\rho}\right)+\gamma\left[\left(1-(1-\theta)^{\rho}\right) J(1,0)+(1-\theta)^{\rho} J(1,1)\right] \\
J(\rho, 1)=s+r\left(1-(1-\theta)^{\rho}\right)+\gamma\left[\left(1-(1-\theta)^{\rho}\right) J(1,0)+(1-\theta)^{\rho} J(1,1)\right]
\end{array}
$$


A limit (cap) on the maximum number of periods that a supplier can go without an inspection is set to make the model finite state space. Also, note that in this problem it is assumed if a supplier is known to be out-of-control, it will be in-control in the following period with probability one. Therefore, to make the problem consistent, the policies in states $(\rho, 1)$ and $(\rho+1,0)$ are set to be "inspect", resulting in $J(\rho+1,0)=$ $J(\rho, 1)$. Note that, having a limit (cap) $\rho$ is not a simplifying assumption as one can set $\rho$ to be a significantly large number so that it does not imply restrictions on optimal policies.

For the case of notation, two functions are defined as follows:

$$
\begin{aligned}
& f(x)=s+r\left(1-(1-\theta)^{x}\right)+\gamma\left[\left(1-(1-\theta)^{x}\right) J(1,0)+(1-\theta)^{x} J(1,1)\right] \\
& g(x)=h\left(1-(1-\theta)^{x}\right)
\end{aligned}
$$

Lemma 1. $J(x, 1)=J(x+1,0)$ for all $x=1,2, \ldots$

Proof: Proof is straightforward by induction on $x$; the number of periods since the last inspection. Note that, by construction $J(\rho+1,0)=J(\rho, 1)$. Assume the lemma is true for all $x=n+1, n+2, \ldots \rho$. Then for $x=n$ there are:

$$
\begin{gathered}
J(n+1,0)=\min \left\{\begin{array}{l}
s+r\left(1-(1-\theta)^{n}\right)+\gamma\left[\left(1-(1-\theta)^{n}\right) J(1,0)+(1-\theta)^{n} J(1,1)\right], \\
h\left(1-(1-\theta)^{n}\right)+\gamma \cdot J(n+2,0)
\end{array}\right. \\
J(n, 1)=\min \left\{\begin{array}{l}
s+r\left(1-(1-\theta)^{n}\right)+\gamma\left[\left(1-(1-\theta)^{n}\right) J(1,0)+(1-\theta)^{n} J(1,1)\right], \\
h\left(1-(1-\theta)^{n}\right)+\gamma \cdot J(n+1,1)
\end{array}\right.
\end{gathered}
$$

Since $J(n+2,0)=J(n+1,1)$ by the inductive hypothesis: $J(n+1,0)=J(n, 1)$.

Due to this lemma, it would be sufficient to prove any result either for states $(x, 0)$ or $(x, 1)$.

Lemma 2. $J(1,0) \leq J(1,1)$ 
Proof: From Equation 4.29 and Lemma 1:

$$
J(1,0) \leq \gamma J(2,0) \leq J(2,0)=J(1,1)
$$

Lemma 3. $J(1,0)=\gamma J(1,1)$

Proof: From Lemma 1, we have $J(2,0)=J(1,1)$ and therefore $s+\gamma J(1,1) \geq$ $\gamma J(2,0)$. So, from Equation 4.29 we have

$$
J(1,0)=\gamma J(2,0)=\gamma J(1,1)
$$

and the optimal decision in state $(1,0)$ is "do not inspect".

Lemma 4. $J(1,1) \leq \frac{s+r \theta}{(1-\gamma)(1+\gamma \theta)}$

Proof: Following Equation 4.32:

$$
\begin{aligned}
J(1,1) & \leq s+r \theta+\gamma[\theta J(1,0)+(1-\theta) J(1,1)] \\
& =s+r \theta+\gamma^{2} \theta J(1,1)+\gamma(1-\theta) J(1,1)
\end{aligned}
$$

where the second inequality is by Lemma 3 . With some algebra, we can easily obtain:

$$
J(1,1) \leq \frac{s+r \theta}{(1-\gamma)(1+\gamma \theta)}
$$

Lemma 5. Assume the function $f(x)$ in Equation 4.33 is defined for all $x \in \mathbf{R}$. Then the function $f(x)$ is either non-increasing and convex or non-decreasing and concave.

Proof: By definition;

$$
\begin{aligned}
f(x) & =s+r\left(1-(1-\theta)^{x}\right)+\gamma\left[\left(1-(1-\theta)^{x}\right) J(1,0)+(1-\theta)^{x} J(1,1)\right] \\
& =s+r\left(1-(1-\theta)^{x}\right)+\gamma^{2}\left(1-(1-\theta)^{x}\right) J(1,1)+\gamma(1-\theta)^{x} J(1,1)
\end{aligned}
$$


The second inequality follows from Lemma 3 . With some algebra we can easily obtain:

$$
f(x)=s+r+\gamma^{2} J(1,1)+\left[\left(\gamma-\gamma^{2}\right) J(1,1)-r\right](1-\theta)^{x}
$$

In the equation above, the only term that depends on $x$ is $(1-\theta)^{x}$ and the term in brackets is constant since $J(1,1)$ only depends only on the parameters $s, r, h, \gamma$ and $\theta$. Therefore depending on the sign of the term in brackets, which is defined by the given parameters in the problem, $f(x)$ can be either convex or concave. To show monotonicity, consider the difference:

$f(x+1)-f(x)=\left[s+r\left(1-(1-\theta)^{x+1}\right)+\gamma\left[\left(1-(1-\theta)^{x+1}\right) J(1,0)+(1-\theta)^{x+1} J(1,1)\right]\right]-$ $\left[s+r\left(1-(1-\theta)^{x}\right)+\gamma\left[\left(1-(1-\theta)^{x}\right) J(1,0)+(1-\theta)^{x} J(1,1)\right]\right]$

Again, algebraically, we can obtain;

$$
f(x+1)-f(x)=\left[\left(\gamma^{2}-\gamma\right) J(1,1)+r\right] \theta(1-\theta)^{x}
$$

Note that, the term in brackets in the above equation is the negative of the term in brackets in Equation 4.35. Therefore, if $\left(\gamma-\gamma^{2}\right) J(1,1)-r \leq 0$ then $f(x)$ is concave and non-decreasing, otherwise it is convex and non-increasing.

In this dissertation, it is tried to prove the results for as general a model as possible. Therefore, no assumptions on the cost parameters such as $s, r$ or $h$ are made. However, the values of these parameters are important to define the optimal policy of the problem. For example, if $h=0$, then there is no penalty for missed detection and therefore, there is no incentive for inspection. As a result, if $h=0$ then the optimal policy would be "do not inspect" with $J(x, y)=0$ for any feasible state $(x, y)$. Therefore, for inspection to be optimal $h$ must be "high enough". The following lemma defines a lower bound on $h$, for which the suppliers would have to be inspected in some future state in the optimal solution.

Lemma 6. Set $h>\frac{s+r \theta}{\theta} \frac{1-\gamma+\gamma \theta}{1+\gamma \theta}$ then there exists $n>0$ such that the optimal action 
is to inspect in state $(n, y)$ for $y=0$ or $y=1$.

Proof: We will show the result only for $y=1$. Assume $h>\frac{s+r \theta}{\theta} \frac{1-\gamma+\gamma \theta}{1+\gamma \theta}$ but the statement does not hold, in other word such an $n$ does not exists. Therefore, it is never optimal to inspect so the optimal actions for all $x>0$ are "do not inspect". This means;

$$
\begin{aligned}
J(1,1) & =h \theta+\gamma J(2,1) \\
& =h \theta+\gamma\left(h\left(1-(1-\theta)^{2}\right)+\gamma J(3,1)\right) \\
& =h \theta+\gamma\left(h\left(1-(1-\theta)^{2}\right)+\gamma\left(h\left(1-(1-\theta)^{3}\right)+\gamma J(4,1)\right)\right) \\
& =\ldots \\
& =h \theta+h \gamma+h \gamma^{2}+h \gamma^{3}+\ldots-h \gamma(1-\theta)^{2}-h \gamma^{2}(1-\theta)^{3}-h \gamma^{3}(1-\theta)^{4}-\ldots \\
& =h \theta+h \gamma\left(1+\gamma+\gamma^{2}+\ldots\right)-h \gamma(1-\theta)^{2}\left[1+\gamma(1-\theta)+\gamma^{2}(1-\theta)^{2}+\ldots\right] \\
& =h \theta+h \frac{\gamma}{1-\gamma}-h \gamma \frac{(1-\theta)^{2}}{1-\gamma(1-\theta)}
\end{aligned}
$$

from Equation 4.32 we have

$$
J(1,1)=\min \{s+r \theta+\gamma[\theta J(1,0)+(1-\theta) J(1,1)], h \theta+\gamma J(2,1)\}
$$

and therefore

$$
\begin{aligned}
J(1,1) & =h \theta+h \frac{\gamma}{1-\gamma}-h \gamma \frac{(1-\theta)^{2}}{1-\gamma(1-\theta)} \\
& \leq s+r \theta+\gamma[\theta J(1,0)+(1-\theta) J(1,1)] \\
& =s+r \theta+\gamma^{2} \theta J(1,1)+\gamma(1-\theta) J(1,1) \\
& =s+r \theta+\left[\gamma^{2} \theta+\gamma(1-\theta)\right]\left(h \theta+h \frac{\gamma}{1-\gamma}-h \gamma \frac{(1-\theta)^{2}}{1-\gamma(1-\theta)}\right)
\end{aligned}
$$

The third equality follows Lemma 3, the fourth equality is obtained by using $J(1,1)=$ $h \theta+h \frac{\gamma}{1-\gamma}-h \gamma \frac{(1-\theta)^{2}}{1-\gamma(1-\theta)}$. With some algebra we can easily obtain $h \leq \frac{s+r \theta}{\theta} \frac{1-\gamma+\gamma \theta}{1+\gamma \theta}$ which contradicts the initial assumption. 
In the following two lemmas, two new functions $\phi^{\prime}(x)=f(x)-g(x)$ and $\phi^{\prime \prime}(x)=$ $f(x)-g(x)-\gamma f(x+1)$ are defined and the conditions under which these functions are monotone are analyzed. These results are then going to be helpful to prove the main result.

Lemma 7. If $h>\frac{s+r \theta}{\theta}$ then $\phi^{\prime}(x)=f(x)-g(x)$ is a decreasing convex function.

Proof:

$$
\begin{gathered}
\phi^{\prime}(x)=f(x)-g(x)=s+r\left(1-(1-\theta)^{x}\right)+ \\
\gamma\left[\left(1-(1-\theta)^{x}\right) J(1,0)+(1-\theta)^{x} J(1,1)\right]-h\left(1-(1-\theta)^{x}\right)= \\
s+r+\gamma^{2} J(1,1)-h+\left[\left(\gamma-\gamma^{2}\right) J(1,1)-r+h\right](1-\theta)^{x}
\end{gathered}
$$

Above, the last equality is obtained by replacing $J(1,0)$ by $\gamma J(1,1)$. As discussed before $J(1,1)$ is a constant function the value of which depends on the parameters given in the problem. Therefore, the first 4 terms in Equation 4.37 are constant. Also, since $h>\frac{s+r \theta}{\theta}$ then $J(1,1) \geq s+r \theta$. To see this consider Equation 4.32:

$$
J(1,1)=\min \{s+r \theta+\gamma[\theta J(1,0)+(1-\theta) J(1,1)], h \theta+\gamma J(2,1)\}
$$

both of the terms in the minimization function above are greater than or equal to $s+r \theta$. Next, we consider the term in brackets:

$$
\left(\gamma-\gamma^{2}\right) J(1,1)-r+h
$$

The only negative term above is - and by assumption $h>\frac{s+r \theta}{\theta}>r$. Therefore, the term in brackets is positive and $\phi^{\prime}(x)$ is a decreasing convex function.

Lemma 8. If $h>\frac{s+r \theta}{\theta}$ then $\phi "(x)=f(x)-g(x)-\gamma f(x+1)$ is a decreasing convex function. 
Proof:

$$
\begin{aligned}
\phi^{\prime \prime}(x)= & f(x)-g(x)-\gamma f(x+1) \\
= & s+r\left(1-(1-\theta)^{x}\right)+\gamma\left[\left(1-(1-\theta)^{x}\right) J(1,0)+(1-\theta)^{x} J(1,1)\right]- \\
& h\left(1-(1-\theta)^{x}\right)- \\
& \gamma *\left(s+r\left(1-(1-\theta)^{x+1}\right)+\gamma\left[\left(1-(1-\theta)^{x+1}\right) J(1,0)+(1-\theta)^{x+1} J(1,1)\right]\right) \\
= & (1-\gamma)\left(s+r+\gamma^{2} J(1,1)\right)-h+ \\
& {[h-r(1-\gamma+\gamma \theta)+\gamma(1-\gamma)(1-\gamma+\gamma \theta) J(1,1)](1-\theta)^{x} }
\end{aligned}
$$

Again, the last equality is obtained by replacing $J(1,0)$ by $\gamma J(1,1)$. As discussed before, the first 2 terms in Equation 4.38 are constant. The terms in bracket can be written as follow:

$$
\begin{aligned}
& h-r(1-\gamma+\gamma \theta)+\gamma(1-\gamma)(1-\gamma+\gamma \theta) J(1,1)= \\
& (h-r)+r \gamma(1-\theta)+\gamma(1-\gamma)(1-\gamma(1-\theta)) J(1,1)
\end{aligned}
$$

Using the assumption $h>\frac{s+r \theta}{\theta}$ it is easy to show the terms given above are positive and therefore $\phi^{\prime \prime}(x)$ is a decreasing convex function.

Lemma 9. Assume $h \geq \frac{s+r \theta}{\theta}$. If the optimal action in state $(x, 1)$ is to inspect the supplier for $x \geq 1$, then the optimal action in state $(x+1,1)$ is also to inspect.

Proof: If it is optimal to inspect in state $(x, 1)$, then;

$$
\begin{aligned}
f(x) & \leq g(x)+\gamma J(x+1,1) \\
& =g(x)+\gamma \cdot \min \{f(x+1), g(x+1)+\gamma J(x+2,1)\} \\
& \leq g(x)+\gamma f(x+1)
\end{aligned}
$$


Using the inequality $f(x) \leq g(x)+\gamma f(x+1)$ and Lemma 7 , there is;

$$
f(x+1) \leq g(x+1)+\gamma f(x+2)
$$

and similarly,

$$
f(x+k) \leq g(x+k)+\gamma f(x+k)
$$

for any $k \in\{1,2, \ldots\}$. Iteratively combining Equations 4.41 and 4.42 for any $k$, we can easily obtain;

$$
\begin{aligned}
f(x+1) \leq & g(x+1)+\gamma f(x+2) \\
\leq & g(x+1)+\gamma g(x+2)+\gamma^{2} f(x+3) \\
\leq & g(x+1)+\gamma g(x+2)+\gamma^{2} g(x+3)+\gamma^{3} f(x+4) \\
& \vdots \\
\leq & g(x+1)+\gamma g(x+2)+\ldots+\gamma^{k-1} g(x+k)+\gamma^{k} f(x+k+1)
\end{aligned}
$$

for any $k \in\{1,2, \ldots\}$. Therefore, if it is optimal to inspect in state $(x, 1)$ then cost of inspection in period $(x+1,1)$ results in lower cost than any other alternative policy such as not inspecting for the next $k \in\{1,2, \ldots\}$ periods and then inspecting. Therefore, it is also optimal to inspect in state $(x+1,1)$.

Lemma 10. The optimal policy of a single-supplier problem is a threshold policy.

Proof: Follows from Lemma 9.

An optimal inspection decision has a simple threshold structure. An optimal policy is: "do not inspect" if the information state $\left(x_{i}, y_{i}\right)$ is behind the threshold. In addition, the optimal decision is: "inspect" if the information state is ahead of the threshold. Figure 4.5 shows an example of threshold policy for a single-supplier. Threshold structure can move to right or left by changing the parameters of the model. 


\begin{tabular}{|c|c|c|c|c|c|c|c|c|c|}
\hline OC & DI & DI & DI & DI & I & I & $\ldots$ & I & I \\
\hline IC & DI & DI & DI & I & I & I & $\ldots$ & I & \\
\hline$y$ & 1 & 2 & 3 & 4 & 5 & 6 & $\ldots$ & $\rho$ & $\rho+1$ \\
\hline
\end{tabular}

Figure 4.5: An example of threshold policy for a single-supplier (DI: Do not inspect, I: Inspect)

\subsection{Linear programming formulation of DP model}

The proposed dynamic programming formulation can be equivalently formulated as a linear programming problem as shown in Equations 4.44 and 4.45 .

$$
\begin{aligned}
\max _{J(\mathbf{z})} & \sum_{\mathbf{z}} \alpha(\mathbf{z}) \cdot J(\mathbf{z}) \\
\text { subject to } & J(\mathbf{z}) \leq c(\mathbf{z}, \mathbf{a})+\sum_{\mathbf{z}^{\prime} \in \Omega(\mathbf{z}, \mathbf{a})} \gamma \cdot \operatorname{Prob}\left\{\mathbf{z}^{\prime} \mid \mathbf{z}, \mathbf{a}\right\} \cdot J\left(\mathbf{z}^{\prime}\right) \quad \forall \mathbf{z}, \mathbf{a}(4.45)
\end{aligned}
$$

Linear programming (LP) approaches for solving dynamic programming formulation have been studied in literature $[$ e.g., 96, 97, 98]. However, the size of a state space increases exponentially when the problem size increases linearly. For instance, Table 4.1 shows the possible states and actions for single supplier.

Table 4.1: Possible states and actions for single supplier

\begin{tabular}{c|c|c}
$\#$ & possible states & possible actions \\
\hline 1 & $(1,0)$ & $(0,1)$ \\
2 & $(1,1)$ & $(0,1)$ \\
3 & $(2,0)$ & $(0,1)$ \\
4 & $(2,1)$ & $(0,1)$ \\
5 & $(3,0)$ & $(0,1)$ \\
6 & $(3,1)$ & $(0,1)$ \\
$\vdots$ & $\vdots$ & $\vdots$ \\
$2 \rho-1$ & $(\rho, 0)$ & $(0,1)$ \\
$2 \rho$ & $(\rho, 1)$ & $(1)$ \\
$2 \rho+1$ & $(\rho+1,0)$ & $(1)$ \\
\hline Totoal & $2 \rho+1$ & $4 \rho$
\end{tabular}


Therefore, considering $k$ suppliers there are:

$$
\begin{aligned}
& \text { Number of decision variables }=\text { Total number of states }=(2 \rho+1)^{k} \\
& \text { Total number of constraints }=(4 p)^{k}
\end{aligned}
$$

For example, Table 4.2 shows the number of decision variables and number of constraints where $k=\{6,7\}$ and $2 \leqslant \rho \leqslant k$.

\begin{tabular}{cc|l|l}
\multicolumn{3}{c}{ Table } & 4.2 : Problem size for a given $k, \rho$ \\
$k$ & $\rho$ & \# of decision variables & \# of constraints \\
\hline 6 & 2 & 15,625 & 262,144 \\
6 & 3 & 117,649 & $2,985,984$ \\
6 & 4 & 531,441 & $16,777,216$ \\
6 & 5 & $1,771,561$ & $64,000,000$ \\
6 & 6 & $4,826,809$ & $191,102,976$ \\
\hline 7 & 2 & 78,125 & $2,097,152$ \\
7 & 3 & 823,543 & $35,831,808$ \\
7 & 4 & $4,782,969$ & $268,435,456$ \\
7 & 5 & $19,487,171$ & $1,280,000,000$ \\
7 & 6 & $62,748,517$ & $4,586,471,424$ \\
7 & 7 & $170,859,375$ & $13,492,928,512$ \\
\hline
\end{tabular}

As can be seen in Table 4.2, there are many decision variables and constraints even given the small size of the problem. An efficient approach to handle this difficulty is to generate an approximation with relatively few variables and constraints.

\subsection{An approximate dynamic programming model}

An exact solution approach may include several hundreds of thousand decision variables and constraints. This would result in a state space too large to be solved in a reasonable amount of time by the exact algorithm (since this is an operational decision, it is assumed that a reasonable time is less than one hour). Methods based on the Decomposition (e.g. column generation, bender decomposition) and Lagrangian Relaxation techniques are not options any more due to the huge size of the problem. A simplified model, however, can greatly reduce computation time while still 
producing effective solutions. An Approximate Dynamic Programming (ADP) model is presented below that approximates the model based on the structure of the single supplier characteristics. Next, some simplification assumptions are outlined to reduce the size of the problem.

\subsubsection{Action reduction-Constraints reduction}

In the original LP (standard form) formulation, there is a constraint for each combination of $\mathbf{z}, \mathbf{a}$. However, part of the possible combinations of $\mathbf{z}, \mathbf{a}$ can be ignored using optimal action policy of single supplier version of problem. Let:

$a_{i}^{\prime}$ : Optimal action for single supplier $i$

$a_{i}^{\prime \prime}$ : Possible actions for multiple case for a given supplier $i$

Table 4.3 summarizes the four different constraint structures considered in this dissertation using single supplier results. 
Table 4.3: Action reduction process

\begin{tabular}{|c|c|}
\hline Policy & Constraint structure \\
\hline Before action reduction & $\begin{array}{l}\forall \mathbf{z} \\
\forall \mathbf{a} \quad \text { If a satisfies cap }(\rho)\end{array}$ \\
\hline Action reduction version 1 . & 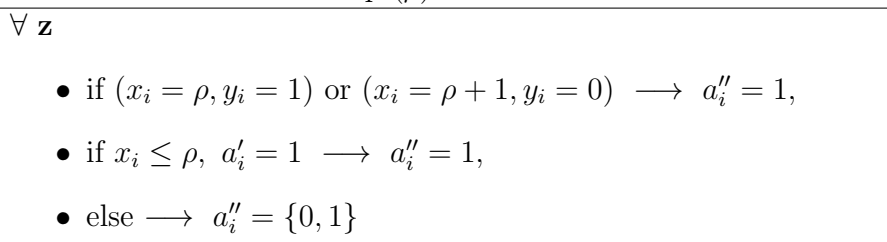 \\
\hline Action reduction version 2 . & $\begin{array}{l}\forall \mathbf{z} \\
\qquad \begin{array}{l}\text { if } \sum_{i=1}^{k}\left(a_{i}^{\prime}\right) \leq n \longrightarrow \text { only consider } \mathbf{a}^{\prime}=\left(a_{1}^{\prime}, a_{2}^{\prime}, \ldots, a_{k}^{\prime}\right), \\
\text { - if } \sum_{i=1}^{k}\left(a_{i}^{\prime}\right)>n: \\
\quad-\quad \text { if }\left(x_{i}=\rho, y_{i}=1\right) \text { or }\left(x_{i}=\rho+1, y_{i}=0\right) \longrightarrow a_{i}^{\prime \prime}=1, \\
\quad-\quad \text { else } \longrightarrow a_{i}^{\prime \prime}=\{0,1\}\end{array}\end{array}$ \\
\hline Action reduction version 3 . & $\begin{array}{l}\forall \mathbf{z} \\
\qquad \begin{array}{l}\text { if } \sum_{i=1}^{k}\left(a_{i}^{\prime}\right) \leq n \rightarrow \text { only consider } \mathbf{a}^{\prime}=\left(a_{1}^{\prime}, a_{2}^{\prime}, \ldots, a_{k}^{\prime}\right), \\
\text { - if } \sum_{i=1}^{k}\left(a_{i}^{\prime}\right)>n: \\
\quad-\quad \text { if } x_{i} \leq \rho, a_{i}^{\prime}=0 \longrightarrow a_{i}^{\prime \prime}=0, \\
\quad-\quad \text { if }\left(x_{i}=\rho, y_{i}=1\right) \text { or }\left(x_{i}=\rho+1, y_{i}=0\right) \longrightarrow a_{i}^{\prime \prime}=1, \\
\quad-\quad \text { else } \longrightarrow a_{i}^{\prime \prime}=\{0,1\}\end{array}\end{array}$ \\
\hline Action reduction version 4 & $\begin{array}{l}\forall \mathbf{z} \\
\qquad \begin{aligned} \bullet & \text { if } \\
& \sum_{i=1}^{k}\left(a_{i}^{\prime}\right) \leq n:, \\
& -\quad \text { if }\left(x_{i}=\rho, y_{i}=1\right) \text { or }\left(x_{i}=\rho+1, y_{i}=0\right) \longrightarrow a_{i}^{\prime \prime}=1, \\
& -\quad \text { if } x_{i} \leq \rho, a_{i}^{\prime}=1 \longrightarrow a_{i}^{\prime \prime}=1, \\
& -\quad \text { else } \longrightarrow a_{i}^{\prime \prime}=\{0,1\} \\
\bullet & \text { if } \sum_{i=1}^{k}\left(a_{i}^{\prime}\right)>n: \\
& -\quad \text { if } x_{i} \leq \rho, a_{i}^{\prime}=0 \longrightarrow a_{i}^{\prime \prime}=0, \\
& -\quad \text { if }\left(x_{i}=\rho, y_{i}=1\right) \text { or }\left(x_{i}=\rho+1, y_{i}=0\right) \longrightarrow a_{i}^{\prime \prime}=1, \\
& -\quad \text { else } \longrightarrow a_{i}^{\prime \prime}=\{0,1\}\end{aligned}\end{array}$ \\
\hline
\end{tabular}

In the numerical experiments, it was realized that proposed action reduction process was not strong enough to reduce a significant number of the constraints. Even only considering one action per state, the number of constraints would be equal to total number of states which is a huge number and unsolvable in large-scale applications. 


\subsubsection{State reduction-Variables reduction}

As mentioned earlier, in the proposed DP formulation, the number of state variables increases exponentially which is intractable in practical scale. Therefore, an effective approach to approximate cost-to-go function with relatively few variables is proposed. As shown in Equation 4.48, an approximation of $J(\mathbf{z})$ (i.e., $\tilde{J}(\mathbf{z})$ ) is developed similar to statistical regression method considering results of optimal value of $J$ for a single supplier.

$$
\tilde{J}(\mathbf{z})=\tilde{J}\left(\left[\begin{array}{c}
z_{1} \\
z_{2} \\
\vdots \\
z_{k}
\end{array}\right]\right)=\lambda_{1} J_{1}^{\prime}\left(z_{1}\right)+\lambda_{2} J_{2}^{\prime}\left(z_{2}\right)+\cdots+\lambda_{k} J_{k}^{\prime}\left(z_{k}\right)+\beta
$$

where, $J_{i}^{\prime}\left(z_{i}\right)$ is optimal value for single supplier $i$ and $\lambda_{i}$ is a new variable. For each supplier $i$, there are $2 \rho+1$ possible states for $z_{i}$ and therefore, $\lambda_{i}$ would be one of the $\left\{\lambda_{i, 1}\right.$ or $\lambda_{i, 2}$ or $\ldots$ or $\left.\lambda_{i, 2 \rho+1}\right\}$. following this approach there is:

$$
\text { Number of decision variables }=(2 \rho+1) k+1
$$


For example if $k=2, \rho=2$ :

$$
\begin{aligned}
& \left\{\begin{array}{c}
z_{1}=(1,0), \lambda_{1}=\lambda_{1,1} \text { or } \\
z_{1}=(1,1), \lambda_{1}=\lambda_{1,2} \text { or } \\
z_{1}=(2,0), \lambda_{1}=\lambda_{1,3} \text { or } \\
z_{1}=(2,1), \lambda_{1}=\lambda_{1,4} \text { or } \\
z_{1}=(3,0), \lambda_{1}=\lambda_{1,5}
\end{array}\right\},\left\{\begin{array}{l}
z_{2}=(1,0), \lambda_{2}=\lambda_{2,1} \text { or } \\
z_{2}=(1,1), \lambda_{2}=\lambda_{2,2} \text { or } \\
z_{2}=(2,0), \lambda_{2}=\lambda_{2,3} \text { or } \\
z_{2}=(2,1), \lambda_{2}=\lambda_{2,4} \text { or } \\
z_{2}=(3,0), \lambda_{2}=\lambda_{2,5}
\end{array}\right\} \\
& \text { if } \left.\left\{\begin{array}{c}
z_{1}=(1,0) \\
z_{2}=(1,0)
\end{array}\right\} \rightarrow \tilde{J}\left(\begin{array}{c}
z_{1} \\
z_{2}
\end{array}\right]\right)=\lambda_{1} J_{1}^{\prime}\left(z_{1}\right)+\lambda_{2} J_{2}^{\prime}\left(z_{2}\right)+\beta \\
& \text { if }\left\{\begin{array}{c}
\left([\mathbf{z})=\tilde{J}\left(\left[\begin{array}{c}
(1,0) \\
(1,0)
\end{array}\right]\right)=\lambda_{1,1} J_{1}^{\prime}(1,0)+\lambda_{2,1} J_{2}^{\prime}(1,0)+\beta(1,0)\right. \\
z_{2}=(1,1)
\end{array}\right\} \rightarrow \tilde{J}(\mathbf{z})=\tilde{J}\left(\left[\begin{array}{c}
(1,0) \\
(1,1)
\end{array}\right]\right)=\lambda_{1,1} J_{1}^{\prime}(1,0)+\lambda_{2,2} J_{2}^{\prime}(1,1)+\beta(5) \\
& \text { if }\left\{\begin{array}{c}
z_{1}=(3,0) \\
z_{2}=(3,0)
\end{array}\right\} \rightarrow \tilde{J}(\mathbf{z})=\tilde{J}\left(\left[\begin{array}{c}
(3,0) \\
(3,0)
\end{array}\right]\right)=\lambda_{1,5} J_{1}^{\prime}(3,0)+\lambda_{2,5} J_{2}^{\prime}(3,0)+\beta(1,5)
\end{aligned}
$$

if $\alpha(\mathbf{z})=1$ :

$$
\begin{aligned}
\mathrm{OF}= & \max _{\tilde{J}(\mathbf{z})} \sum_{\mathbf{z}} \tilde{J}(\mathbf{z}) \\
= & \left\{\lambda_{1,1} J_{1}^{\prime}(1,0)+\lambda_{2,1} J_{2}^{\prime}(1,0)+\beta\right\}+\left\{\lambda_{1,1} J_{1}^{\prime}(1,0)+\lambda_{2,1} J_{2}^{\prime}(1,0)+\beta\right\}+ \\
& \cdots+\left\{\lambda_{1,5} J_{1}^{\prime}(3,0)+\lambda_{2,5} J_{2}^{\prime}(3,0)+\beta\right\} \\
= & 5\left[\lambda_{1,1} J_{1}^{\prime}(1,0)+\lambda_{1,2} J_{1}^{\prime}(1,1)+\lambda_{1,3} J_{1}^{\prime}(2,0)+\lambda_{1,4} J_{1}^{\prime}(2,1)+\lambda_{1,5} J_{1}^{\prime}(3,0)\right]+ \\
& 5\left[\lambda_{2,1} J_{2}^{\prime}(1,0)+\lambda_{2,2} J_{2}^{\prime}(1,1)+\lambda_{2,3} J_{2}^{\prime}(2,0)+\lambda_{2,4} J_{2}^{\prime}(2,1)+\lambda_{2,5} J_{2}^{\prime}(3,0)\right]+ \\
& 5^{2} \beta
\end{aligned}
$$


subject to:

$$
\begin{aligned}
& \tilde{J}(\mathbf{z}) \leq c(\mathbf{z}, \mathbf{a})+\sum_{\mathbf{z}^{\prime} \in \Omega(\mathbf{z}, \mathbf{a})} \gamma \cdot \operatorname{Prob}\left\{\mathbf{z}^{\prime} \mid \mathbf{z}, \mathbf{a}\right\} \cdot \tilde{J}\left(\mathbf{z}^{\prime}\right) \quad \forall \mathbf{z}, \mathbf{a} \\
& \text { if }\left\{\begin{array}{c}
z_{1}=(1,0) \\
z_{2}=(1,0) \\
\mathbf{a}=(0,0)
\end{array}\right\} \rightarrow \tilde{J}\left(\left[\begin{array}{l}
(1,0) \\
(1,0)
\end{array}\right]\right) \leq c\left(\left[\begin{array}{l}
(1,0) \\
(1,0)
\end{array}\right],(0,0)\right)+ \\
& \gamma \cdot 1 \cdot \tilde{J}\left(\left[\begin{array}{c}
(2,0) \\
(2,0)
\end{array}\right]\right) \\
& \text { if }\left\{\begin{array}{c}
z_{1}=(1,0) \\
z_{2}=(1,0) \\
\mathbf{a}=(0,1)
\end{array}\right\} \rightarrow \tilde{J}\left(\left[\begin{array}{l}
(1,0) \\
(1,0)
\end{array}\right]\right) \leq c\left(\left[\begin{array}{l}
(1,0) \\
(1,0)
\end{array}\right],(0,1)\right)+ \\
& \gamma \cdot 1 \cdot \tilde{J}\left(\left[\begin{array}{l}
(2,0) \\
(1,1)
\end{array}\right]\right) \\
& \vdots \\
& \text { if }\left\{\begin{array}{l}
z_{1}=(3,0) \\
z_{2}=(3,0) \\
\mathbf{a}=(1,1)
\end{array}\right\} \rightarrow \tilde{J}\left(\left[\begin{array}{l}
(3,0) \\
(3,0)
\end{array}\right]\right) \leq c\left(\left[\begin{array}{l}
(3,0) \\
(3,0)
\end{array}\right],(1,1)\right)+ \\
& \gamma\left(1-\theta_{1}\right)^{2}\left(1-\theta_{2}\right)^{2} \cdot \tilde{J}\left(\left[\begin{array}{l}
(1,1) \\
(1,1)
\end{array}\right]\right)+ \\
& \gamma\left(1-\theta_{1}\right)^{2}\left(1-\left(1-\theta_{2}\right)^{2}\right) \cdot \tilde{J}\left(\left[\begin{array}{c}
(1,1) \\
(1,0)
\end{array}\right]\right)+ \\
& \gamma\left(1-\left(1-\theta_{1}\right)^{2}\right)\left(1-\theta_{2}\right)^{2} \tilde{J}\left(\left[\begin{array}{c}
(1,0) \\
(1,1)
\end{array}\right]\right)+ \\
& \gamma\left(1-\left(1-\theta_{1}\right)^{2}\right)\left(1-\left(1-\theta_{2}\right)^{2}\right) \tilde{J}\left(\left[\begin{array}{c}
(1,0) \\
(1,0)
\end{array}\right]\right)
\end{aligned}
$$


By using Equation 4.48, the previous constraints can be rewritten as follows:

$$
\begin{aligned}
& \lambda_{1,1} J_{1}^{\prime}(1,0)+\lambda_{2,1} J_{2}^{\prime}(1,0)+\beta \leq c\left(\left[\begin{array}{l}
(1,0) \\
(1,0)
\end{array}\right],(0,0)\right)+ \\
& \gamma\left(\lambda_{1,3} J_{1}^{\prime}(2,0)+\lambda_{2,3} J_{2}^{\prime}(2,0)+\beta\right) \\
& \lambda_{1,1} J_{1}^{\prime}(1,0)+\lambda_{2,1} J_{2}^{\prime}(1,0)+\beta \leq c\left(\left[\begin{array}{l}
(1,0) \\
(1,0)
\end{array}\right],(0,1)\right)+ \\
& \gamma\left(\lambda_{1,3} J_{1}^{\prime}(2,0)+\lambda_{2,1} J_{2}^{\prime}(1,1)+\beta\right) \\
& \quad \vdots \\
& \lambda_{1,5} J_{1}^{\prime}(3,0)+\lambda_{2,5} J_{2}^{\prime}(3,0)+\beta \leq c\left(\left[\begin{array}{c}
(3,0) \\
(3,0)
\end{array}\right],(1,1)\right)+ \\
& \gamma\left(1-\theta_{1}\right)^{2}\left(1-\theta_{2}\right)^{2} \cdot\left(\lambda_{1,2} J_{1}^{\prime}(1,1)+\lambda_{2,2} J_{2}^{\prime}(1,1)+\beta\right)+ \\
& \gamma\left(1-\theta_{1}\right)^{2}\left(1-\left(1-\theta_{2}\right)^{2}\right) \cdot\left(\lambda_{1,2} J_{1}^{\prime}(1,1)+\lambda_{2,1} J_{2}^{\prime}(1,0)+\beta\right)+ \\
& \gamma\left(1-\left(1-\theta_{1}\right)^{2}\right)\left(1-\theta_{2}\right)^{2} \cdot\left(\lambda_{1,1} J_{1}^{\prime}(1,0)+\lambda_{2,2} J_{2}^{\prime}(1,1)+\beta\right)+ \\
& \gamma\left(1-\left(1-\theta_{1}\right)^{2}\right)\left(1-\left(1-\theta_{2}\right)^{2}\right)\left(\lambda_{1,1} J_{1}^{\prime}(1,0)+\lambda_{2,1} J_{2}^{\prime}(1,0)+\beta\right)
\end{aligned}
$$

In the numerical experiments it was realized that $\tilde{J}(\mathbf{z})$ is a strong approximation function of $J(\mathbf{z})$. Also, using this approach, the number of the variables from polynomial form $\left((2 \rho+1)^{k}\right)$ in the original LP can be significantly reduced to the linear form $((2 \rho+1) k+1)$ in ADP. However, the number of the constraints is still huge and likely unsolvable in large-scale applications.

\subsubsection{Extreme states method - State and action reduction}

In this section, an effective approach to reduce the overall size of the problem while retaining a fairly good solution is discussed. This approach is based on use of $\tilde{J}(\mathbf{z})$ instead of $J(\mathbf{z})$, and incorporating subset of the constraints. The Extreme-states method computational procedure is implemented in the following steps. 
Step 1: Constraints reduction using subset of possible states (extreme states):

- for each supplier $i$, consider all possible states $((1,0),(1,1),(2,0),(2,1)$, $\cdots,(\rho+1,0))$ for supplier $i$, but fixed all the remaining suppliers in state $(\rho+1,0)$.

- ignore all the remaining possible states from constraints

Step 2: Constraints reduction using subset of possible actions:

- For each possible state of Step 1, action will be fixed based on the optimal action of single supplier version.

Step 3: Variables reduction using $\tilde{J}(\mathbf{z})$ instead of $J(\mathbf{z})$.

Following this approach there are:

$$
\begin{aligned}
& \text { Number of decision variables }=(2 p+1) k+1 \\
& \text { Total number of constraints }=(2 p) k+1
\end{aligned}
$$

For example if $k=2, \rho=2$ :

$$
\left(\left\{\begin{array}{l}
z_{1}=(1,0) \text { or } \\
z_{1}=(1,1) \text { or } \\
z_{1}=(2,0) \text { or } \\
z_{1}=(2,1) \text { or } \\
z_{1}=(3,0)
\end{array}\right\}, z_{2}=(3,0)\right) \text { or }\left(z_{1}=(3,0),\left\{\begin{array}{l}
z_{2}=(1,0) \text { or } \\
z_{2}=(1,1) \text { or } \\
z_{2}=(2,0) \text { or } \\
z_{2}=(2,1) \text { or } \\
z_{2}=(3,0)
\end{array}\right\}\right)
$$

In order to prevent unbounded solution, an upper bound (UB) value for $\lambda_{i, j}, \beta$ can be imposed. Initially, it is started with a large UB value and reduced iteratively until all the dual values are equal to zero (i.e., all the constraints are binding). For example, Table 4.4 shows results of Dual and Slack values in case where $k=2, n=2, \theta=$ 
$[0.15,0.15], s=200, \bar{s}=250, r=[350,350], h=[700,700], \gamma=0.95, \rho=2$.

Table 4.4: Dual and Slack values in small example

\begin{tabular}{|c|c|c|c|c|c|c|c|c|c|c|}
\hline \multirow{2}{*}{ Constraint } & \multicolumn{2}{|c|}{$\mathrm{UB}=1.4$} & \multicolumn{2}{|c|}{$\mathrm{UB}=1.2$} & \multicolumn{2}{|c|}{$\mathrm{UB}=1.0$} & \multicolumn{2}{|c|}{$\mathrm{UB}=0.8$} & \multicolumn{2}{|c|}{$\mathrm{UB}=0.6$} \\
\hline & Dual & Slack & Dual & Slack & Dual & Slack & Dual & Slack & Dual & Slack \\
\hline$Z=([1,0],[3,0]), a=(0,1)$ & 4.5 & 0 & 4.5 & 0 & 4.5 & 0 & 0 & 59.4 & 0 & 118.8 \\
\hline$Z=([1,1],[3,0]), a=(0,1)$ & 0 & 98.5 & 0 & 49.3 & 0 & 0.04 & 0 & 80.39 & 0 & 160.8 \\
\hline$Z=([2,0],[3,0]), a=(0,1)$ & 9.3 & 0 & 9.3 & 0 & 9.3 & 0 & 0 & 80.4 & 0 & 160.82 \\
\hline$Z=([2,1],[3,0]), a=(1,1)$ & 0 & 21.7 & 0 & 10.9 & 0 & 0.04 & 0 & 118.81 & 0 & 237.7 \\
\hline$Z=([3,0],[1,0]), a=(1,0)$ & 4.5 & 0 & 4.5 & 0 & 4.5 & 0 & 0 & 59.4 & 0 & 118.82 \\
\hline$Z=([3,0],[1,1]), a=(1,0)$ & 0 & 98.5 & 0 & 49.3 & 0 & 0.04 & 0 & 80.39 & 0 & 160.8 \\
\hline$Z=([3,0],[2,0]), a=(1,0)$ & 9.3 & 0 & 9.3 & 0 & 9.3 & 0 & 0 & 80.4 & 0 & 160.8 \\
\hline$Z=([3,0],[2,1]), a=(1,1)$ & 0 & 21.7 & 0 & 10.9 & 0 & 0.04 & 0 & 118.81 & 0 & 237.7 \\
\hline$Z=([3,0],[3,0]), a=(1,1)$ & 0 & 281.17 & 0 & 140.65 & 0 & 0.14 & 0 & 118.81 & 0 & 237.67 \\
\hline
\end{tabular}

As it is shown in 4.4, when $\mathrm{UB} \geq 1.0$, there is at least one dual value unequal to zero (or there is at least one slack value equal to zero). However, when $\mathrm{UB} \leq 0.8$, all the dual values are equal to zero (or all the slack values are unequal to zero). The range $[0.8,1]$ can be further analyzed to further refine the range for UB. In this example, the range [0.9998, 0.9999] has the best upper-bound value.

The best UB can be calculated without any optimization. In other words, the standard LP version of the Extreme-states model can be expressed in matrix form as $\max \left\{c^{T} \mathbf{x} \mid A \mathbf{x} \leq b, \mathbf{x} \leq \mathrm{UB} \cdot 1\right\}$ where $x=\left[\lambda_{1}, \lambda_{2}, \cdots, \lambda_{k}, \beta\right]^{T}$, and $A$ is a matrix of coefficients, and $b$ is right hand side of the constraints. Due to the fact that $A(\mathrm{UB} .1) \leq b$ and this a maximization problem, the best $\mathrm{UB}$ would be the highest value of UB where all inequalities are satisfied.

In the numerical experiments, it was realized that by using this approximation approach the value of $J(\mathbf{z})$ can be estimated within an acceptable gap difference.

\subsubsection{An extended of the Extreme-states method}

In this section, the approximation approach based on the Extreme-states method is extended and another approach to reduce gap difference is discussed. The Extremestates method is used as a base model and then additional constraints are added. These constraints should have certain characteristics. The following steps show the 
details of extended of the Extreme-states scenario approach.

Step 1: Consider Extreme-states model: $\max \left\{c^{T} \mathbf{x} \mid A \mathbf{x} \leq b, \mathbf{x} \leq \mathrm{UB} \cdot 1\right\}$

- find the best UB for Extreme-states model,

- $\mathrm{UB} \rightarrow \mathrm{UB}+\varepsilon$ where, $\varepsilon$ is a really small positive number.

- consider updated UB as a parameter in the model and solve the model

Step 2: $\forall c \in\{$ Constraints set $\}:$ If $\exists c \mid c \cdot$ slack $=0$

- consider all the $\mathbf{z}$ included in the constraint $c$,

- for each possible $\mathbf{z}$, consider optimal a from single supplier version.

Step 3: Add all corresponding constraints $(\mathbf{z}, \mathbf{a})$ to the $\{$ Constraints set $\}$.

- find the best UB for updated model,

$-\mathrm{UB} \rightarrow \mathrm{UB}+\varepsilon$ where, $\varepsilon$ is a really small positive number.

- consider updated UB in the updated model and solve the model.

Step 4: move to the Step 2.

Step 5: Stop if $\nexists c \mid c \cdot$ slack $=0$.

In other words, this approach uses all the benefits of Extreme-states method and tries to incorporate a part of the most effective remaining constraints to the model. The numerical experiments indicate that by using this approximation approach the better estimation of $J(\mathbf{z})$ and/or better action policy could be found.

\subsection{Numerical Results}

In this section, the computational results are summarized. A workstation with two 3.066 GHz Quad-Core Intel Xeon processors and 32 GB memory was used to run all the experiments. The Original-LP model, and approximation procedures were 
implemented in the Python language. The Gurobi Optimizer 5.1.0 was used to solve all the optimization problems.

Table 4.5 shows the comparison results of exact method vs. two proposed approximation methods. The exact method is unable to solve the problem in acceptable time when $k=5, \rho \geq 5$ while, both of approximation approaches can deliver high quality results very fast (in the experiments of Table 4.5: $\theta_{i}=0.15, s=\$ 1000, \bar{s}=$ $\left.\$ 1500, n=\lfloor k / 2\rfloor, r_{i}=\$ 2000, h_{i} \in[\$ 1500, \$ 4000], \gamma=0.9\right)$. The average absolute difference (aad) between $J^{*}(\mathbf{z}), \tilde{J}(\mathbf{z})$ is also shown. In other words,

$$
a a d=\sum_{i=1}^{(2 \rho+1)^{k}}\left|J^{*}\left(\mathbf{z}_{i}\right)-\tilde{J}\left(\mathbf{z}_{i}\right)\right| /(2 \rho+1)^{k}
$$

where, $J^{*}\left(\mathbf{z}_{i}\right), \tilde{J}\left(\mathbf{z}_{i}\right)$ are calculated based on exact and approximation approaches, respectively.

Table 4.5: Exact method vs. Approximation method

\begin{tabular}{|cc|c|cc|cc|}
\hline & & Exact method & \multicolumn{4}{|c|}{ Approximation method } \\
\cline { 2 - 7 } & Original-LP & \multicolumn{2}{|c|}{ Extreme-states method } & \multicolumn{2}{|c|}{ Extended extreme-states method } \\
\hline 2 & $\rho$ & time (sec) & time (sec) & aad (\%) & time (sec) & aad (\%) \\
2 & 2 & 0.14 & 0.07 & $3.52 \%$ & 0.31 & $3.55 \%$ \\
2 & 4 & 0.17 & 0.12 & $0.44 \%$ & 0.32 & $0.46 \%$ \\
2 & 5 & 0.25 & 0.12 & $0.40 \%$ & 0.23 & $0.40 \%$ \\
2 & 6 & 0.31 & 0.12 & $0.40 \%$ & 0.24 & $0.40 \%$ \\
\hline 3 & 2 & 0.46 & 0.14 & $0.47 \%$ & 0.27 & $0.47 \%$ \\
3 & 3 & 0.37 & 0.12 & $4.75 \%$ & 0.35 & $8.15 \%$ \\
3 & 4 & 0.88 & 0.12 & $10.44 \%$ & 0.35 & $1.22 \%$ \\
3 & 5 & 3.12 & 0.14 & $9.66 \%$ & 0.40 & $1.08 \%$ \\
3 & 6 & 6.61 & 0.21 & $8.91 \%$ & 0.71 & $1.15 \%$ \\
\hline 4 & 2 & 2.55 & 0.23 & $8.17 \%$ & 0.81 & $1.35 \%$ \\
4 & 3 & 16.69 & 0.22 & $7.39 \%$ & 0.42 & $2.17 \%$ \\
4 & 4 & 64.88 & 0.25 & $7.33 \%$ & $0.4 \%$ & $0.26 \%$ \\
4 & 5 & 245.94 & 0.33 & $6.70 \%$ & 0.77 & $0.28 \%$ \\
4 & 6 & 823.31 & 0.33 & $6.18 \%$ & 0.85 & $0.38 \%$ \\
\hline 5 & 2 & 38.58 & 0.22 & $9.24 \%$ & 0.67 & $0.51 \%$ \\
5 & 3 & 679 & 0.3 & $12.20 \%$ & 0.80 & $4.73 \%$ \\
5 & 4 & 7531.91 & 0.41 & $11 \%$ & 1.04 & $0.58 \%$ \\
5 & 5 & 58738.25 & 0.71 & $10 \%$ & 1.13 & $0.67 \%$ \\
5 & 6 & - & 0.75 & - & 1.42 & $0.85 \%$ \\
\hline
\end{tabular}


As it is shown in Table 4.5, the performance of both proposed approximation approaches is acceptable. However, the performance of the extended Extreme-states method is better than the Extreme-states method. The extended Extreme-states approximation approach can estimate the value of $J^{*}(\mathbf{z})$ with very low gap difference (less than $2 \%$ gap in most of cases).

Two proposed approximation approaches are exercised to provide insights about how key parameters $\left(\bar{s}, r_{i}, h_{i}\right)$ impact the obtained solutions. In this regard, initially, it is assumed that $k=4, n=2, \theta_{i}=0.15, s=\$ 1000, \bar{s}=\$ 1500, r_{i}=\$ 2000, h=$ $[\$ 1500, \$ 2500, \$ 3000, \$ 3000], \quad \gamma=0.9$. By using these parameters, both proposed approximation approaches are solved to make inspection action decision. First, all the parameters are fixed as the mentioned values above except for the value of $\bar{s}$. The value of $\bar{s}$ is changed from $\$ 1,500$ to $\$ 3,000$ (i.e., double of initial value) and then, both proposed approximation approaches are solved. Table 4.6 shows comparison between both approximation approaches considering aad.

Table 4.6: Comparison between approximation approaches changing $\bar{s}$

\begin{tabular}{|cc|cc|cc|}
\hline & & \multicolumn{2}{|c|}{ Extreme-states method } & \multicolumn{2}{c|}{ Extended extreme-states method } \\
\cline { 3 - 6 } & $\bar{s}=\$ 1500$ & $\bar{s}=\$ 3000$ & $\bar{s}=\$ 1500$ & $\bar{s}=\$ 3000$ \\
\hline $\mathrm{k}$ & $\rho$ & $\operatorname{aad}(\%)$ & $\operatorname{aad}(\%)$ & $\operatorname{aad}(\%)$ & $\operatorname{aad}(\%)$ \\
\hline 4 & 2 & $7.39 \%$ & $34.99 \%$ & $2.17 \%$ & $2.95 \%$ \\
4 & 3 & $8.04 \%$ & $32.51 \%$ & $0.26 \%$ & $0.59 \%$ \\
4 & 4 & $7.33 \%$ & $29.92 \%$ & $0.28 \%$ & $0.43 \%$ \\
4 & 5 & $6.70 \%$ & $27.83 \%$ & $0.38 \%$ & $0.48 \%$ \\
4 & 6 & $6.18 \%$ & $26.15 \%$ & $0.51 \%$ & $0.60 \%$ \\
\hline
\end{tabular}

As shown in 4.6, almost optimal policy can be found by extended extreme state method. In other words, this approach utilizes capacity very efficiently and there is not much required inspection beyond the capacity limit. Therefore, by increasing $\bar{s}$ from $\$ 1,500$ to $\$ 3,000$, there is not any big differences in the aad value (i.e., this approach is not sensitive in to changes of $\bar{s}$ ). In contrast, extreme state method is not very efficient and its output is very sensitive to changes of $\bar{s}$. 
Same procedure is used for analyzing the effect of $r, h$ on the performance of approximation approaches. Table 4.7 and Table 4.8 summarize the effect of $r$ and $s$ on the results of two proposed approximation approaches. In Table 4.8, it is assumed that $h_{1}=[\$ 1500, \$ 2500, \$ 3000, \$ 3000]$, and $h_{2}=[\$ 3000, \$ 5000, \$ 6000, \$ 6000]$. The results of numerical experiments indicate that, almost optimal policy can be found by extended extreme state method and this approximation approach is not sensitive to parameters. In contrast, extreme state method is less efficient and more sensitive to parameters compared to extended extreme state method.

Table 4.7: Comparison between approximation approaches changing $r$

\begin{tabular}{|cc|cc|cc|}
\hline & & \multicolumn{2}{|c|}{ Extreme-states method } & \multicolumn{2}{c|}{ Extended extreme-states method } \\
\cline { 2 - 6 } & & $r_{i}=\$ 2000$ & $r_{i}=\$ 4000$ & $r_{i}=\$ 2000$ & $r_{i}=\$ 4000$ \\
\hline $\mathrm{k}$ & $\rho$ & $\operatorname{aad}(\%)$ & $\operatorname{aad}(\%)$ & $\operatorname{aad}(\%)$ & $\operatorname{aad}(\%)$ \\
\hline 4 & 2 & $7.39 \%$ & $5.39 \%$ & $2.17 \%$ & $1.85 \%$ \\
4 & 3 & $8.04 \%$ & $5.76 \%$ & $0.26 \%$ & $0.15 \%$ \\
4 & 4 & $7.33 \%$ & $5.09 \%$ & $0.28 \%$ & $0.14 \%$ \\
4 & 5 & $6.70 \%$ & $4.58 \%$ & $0.38 \%$ & $0.18 \%$ \\
4 & 6 & $6.18 \%$ & $4.21 \%$ & $0.51 \%$ & $0.21 \%$ \\
\hline
\end{tabular}

Table 4.8: Comparison between approximation approaches changing $h$

\begin{tabular}{|cc|cc|cc|}
\hline & & \multicolumn{2}{|c|}{ Extreme-states method } & \multicolumn{2}{c|}{ Extended extreme-states method } \\
\cline { 3 - 6 } & & $h=h_{1}$ & $h=h_{2}$ & $h=h_{1}$ & $h=h_{2}$ \\
\hline $\mathrm{k}$ & $\rho$ & $\operatorname{aad}(\%)$ & $\operatorname{aad}(\%)$ & $\operatorname{aad}(\%)$ & $\operatorname{aad}(\%)$ \\
\hline 4 & 2 & $7.39 \%$ & $8.33 \%$ & $2.17 \%$ & $0.61 \%$ \\
4 & 3 & $8.04 \%$ & $7.37 \%$ & $0.26 \%$ & $0.62 \%$ \\
4 & 4 & $7.33 \%$ & $6.47 \%$ & $0.28 \%$ & $0.86 \%$ \\
4 & 5 & $6.70 \%$ & $5.74 \%$ & $0.38 \%$ & $1.10 \%$ \\
4 & 6 & $6.18 \%$ & $5.19 \%$ & $0.51 \%$ & $1.28 \%$ \\
\hline
\end{tabular}

Simulation results. A simulation model is provided to compare the performance of inspection policy obtained from the proposed approximate DP algorithm with other Baseline policies. The baseline policy considers periodically inspection of suppliers without considering capacity, underling supply chain structure, and stochasticity. In other words, the Baseline policy requires the inspection of suppliers every $t$ periods $(t \in\{2,3,4,5,6\})$. 
Approximately similar baseline policy is being used by several organizations. For example, The FDA requires inspection of pharmaceutical suppliers at least once every two years [44]. In addition, the Maine Department of Transportation (MDOT) inspects all bridges every 24 months [81].

Further, the simulation computational procedure is implemented as follows:

Step 1: Initialization $(\mathrm{t}=1)$ : suppose $S_{A}=$ actual-state, $S_{D P}=$ DP-state

$$
\begin{aligned}
& t=1 \quad t=1
\end{aligned}
$$

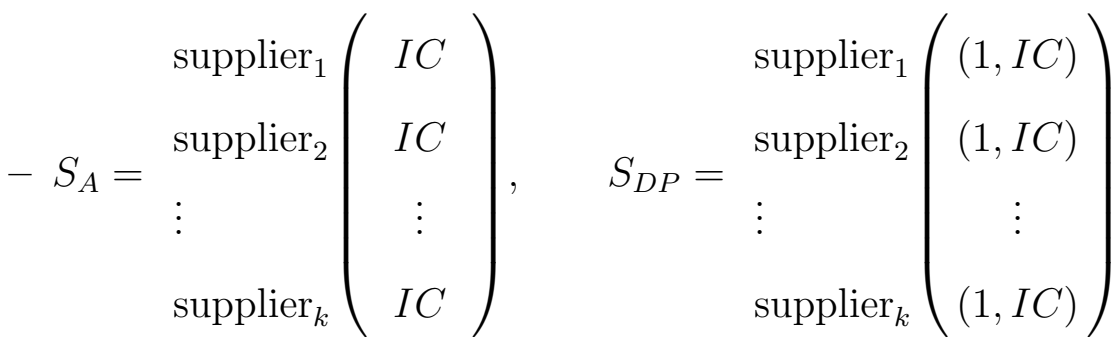

Step 2: Iteration: $\forall t \in\{2,3, \cdots$, Number of periods $\}$ :

- randomly sample $S_{A}$

$*$ if $\operatorname{Rand}() \leqq \theta_{i}: S_{A}(i)=\mathrm{OC} \quad \forall i \in\{1,2, \cdots, k\}$

* else: $S_{A}(i)=\mathrm{IC}$.

- $O A=$ Optimal-Action $\left(S_{D P}\right)$, update $S_{D P}$,

- calculate immediate cost $\left(S_{A}, O A\right)$

$-t=t+1$

Step 3: End: calculate the present value and return it.

Table 4.9 shows the comparison results of Approximation method vs. Baseline policy. In order to get the most accurate results, each simulation run is repeated 1,000 times and average present value of 1,000 independent simulation runs, is used for comparison (in the experiments, $\theta_{i}=0.15, s=\$ 1000, \bar{s}=\$ 1500, n \cong\lfloor k / 2\rfloor, r_{i}=\$ 2000, h_{i} \in$ $[\$ 1500, \$ 4000], \gamma=0.9)$. 
Table 4.9: Simulation results: Approximation method vs. Baseline policy

\begin{tabular}{|c|c|c|c|c|c|c|c|c|}
\hline \multirow[b]{2}{*}{$\mathrm{k}$} & \multirow[b]{2}{*}{$\rho$} & \multirow[b]{2}{*}{$\begin{array}{l}\text { Number } \\
\text { of periods }\end{array}$} & Aproximation & \multicolumn{5}{|c|}{ Baseline policy } \\
\hline & & & $\begin{array}{l}\text { Extended } \\
\text { extreme-states }\end{array}$ & $\begin{array}{l}\text { Inspect every } \\
2 \text { periods }\end{array}$ & $\begin{array}{l}\text { Inspect every } \\
3 \text { periods }\end{array}$ & $\begin{array}{l}\text { Inspect every } \\
4 \text { periods }\end{array}$ & $\begin{array}{l}\text { Inspect every } \\
5 \text { periods }\end{array}$ & $\begin{array}{l}\text { Inspect every } \\
6 \text { periods }\end{array}$ \\
\hline 2 & 2 & 1000 & $\$ 17,229$ & $\$ 21,043$ & $\$ 18,702$ & $\$ 18,181$ & $\$ 18,675$ & $\$ 18,914$ \\
\hline 2 & 3 & 1000 & $\$ 16,211$ & $\$ 21,043$ & $\$ 18,702$ & $\$ 18,181$ & $\$ 18,675$ & $\$ 18,914$ \\
\hline 2 & 4 & 1000 & $\$ 15,098$ & $\$ 21,043$ & $\$ 18,702$ & $\$ 18,181$ & $\$ 18,675$ & $\$ 18,914$ \\
\hline 2 & 5 & 1000 & $\$ 14,715$ & $\$ 21,043$ & $\$ 18,702$ & $\$ 18,181$ & $\$ 18,675$ & $\$ 18,914$ \\
\hline 2 & 6 & 1000 & $\$ 14,758$ & $\$ 21,043$ & $\$ 18,702$ & $\$ 18,181$ & $\$ 18,675$ & $\$ 18,914$ \\
\hline 3 & 2 & 1000 & $\$ 28,456$ & $\$ 33,522$ & $\$ 30,098$ & $\$ 29,708$ & $\$ 30,451$ & $\$ 31,271$ \\
\hline 3 & 3 & 1000 & $\$ 24,812$ & $\$ 33,522$ & $\$ 30,098$ & $\$ 29,708$ & $\$ 30,451$ & $\$ 31,271$ \\
\hline 3 & 4 & 1000 & $\$ 24,138$ & $\$ 33,522$ & $\$ 30,098$ & $\$ 29,708$ & $\$ 30,451$ & $\$ 31,271$ \\
\hline 3 & 5 & 1000 & $\$ 23,793$ & $\$ 33,522$ & $\$ 30,098$ & $\$ 29,708$ & $\$ 30,451$ & $\$ 31,271$ \\
\hline 3 & 6 & 1000 & $\$ 24,101$ & $\$ 33,522$ & $\$ 30,098$ & $\$ 29,708$ & $\$ 30,451$ & $\$ 31,271$ \\
\hline 4 & 2 & 1000 & $\$ 35,781$ & $\$ 43,584$ & $\$ 40,093$ & $\$ 40,355$ & $\$ 40,995$ & $\$ 43,532$ \\
\hline 4 & 3 & 1000 & $\$ 33,453$ & $\$ 43,584$ & $\$ 40,093$ & $\$ 40,355$ & $\$ 40,995$ & $\$ 43,532$ \\
\hline 4 & 4 & 1000 & $\$ 33,000$ & $\$ 43,584$ & $\$ 40,093$ & $\$ 40,355$ & $\$ 40,995$ & $\$ 43,532$ \\
\hline 4 & 5 & 1000 & $\$ 33,204$ & $\$ 43,584$ & $\$ 40,093$ & $\$ 40,355$ & $\$ 40,995$ & $\$ 43,532$ \\
\hline 4 & 6 & 1000 & $\$ 32,990$ & $\$ 43,584$ & $\$ 40,093$ & $\$ 40,355$ & $\$ 40,995$ & $\$ 43,532$ \\
\hline 5 & 2 & 1000 & $\$ 47,965$ & $\$ 56,607$ & $\$ 52,574$ & $\$ 53,085$ & $\$ 55,772$ & $\$ 58,059$ \\
\hline 5 & 3 & 1000 & $\$ 42,974$ & $\$ 56,607$ & $\$ 52,574$ & $\$ 53,085$ & $\$ 55,772$ & $\$ 58,059$ \\
\hline 5 & 4 & 1000 & $\$ 42,368$ & $\$ 56,607$ & $\$ 52,574$ & $\$ 53,085$ & $\$ 55,772$ & $\$ 58,059$ \\
\hline 5 & 5 & 1000 & $\$ 42,384$ & $\$ 56,607$ & $\$ 52,574$ & $\$ 53,085$ & $\$ 55,772$ & $\$ 58,059$ \\
\hline 5 & 6 & 1000 & $\$ 42,346$ & $\$ 56,607$ & $\$ 52,574$ & $\$ 53,085$ & $\$ 55,772$ & $\$ 58,059$ \\
\hline 6 & 2 & 1000 & $\$ 54,087$ & $\$ 65,701$ & $\$ 60,323$ & $\$ 60,711$ & $\$ 62,834$ & $\$ 66,052$ \\
\hline 6 & 3 & 1000 & $\$ 49,459$ & $\$ 65,701$ & $\$ 60,323$ & $\$ 60,711$ & $\$ 62,834$ & $\$ 66,052$ \\
\hline 6 & 4 & 1000 & $\$ 49,454$ & $\$ 65,701$ & $\$ 60,323$ & $\$ 60,711$ & $\$ 62,834$ & $\$ 66,052$ \\
\hline 6 & 5 & 1000 & $\$ 49,253$ & $\$ 65,701$ & $\$ 60,323$ & $\$ 60,711$ & $\$ 62,834$ & $\$ 66,052$ \\
\hline 6 & 6 & 1000 & $\$ 49,386$ & $\$ 65,701$ & $\$ 60,323$ & $\$ 60,711$ & $\$ 62,834$ & $\$ 66,052$ \\
\hline 7 & 2 & 1000 & $\$ 65,563$ & $\$ 78,124$ & $\$ 71,318$ & $\$ 71,567$ & $\$ 74,148$ & $\$ 76,409$ \\
\hline 7 & 3 & 1000 & $\$ 58,380$ & $\$ 78,124$ & $\$ 71,318$ & $\$ 71,567$ & $\$ 74,148$ & $\$ 76,409$ \\
\hline 7 & 4 & 1000 & $\$ 57,278$ & $\$ 78,124$ & $\$ 71,318$ & $\$ 71,567$ & $\$ 74,148$ & $\$ 76,409$ \\
\hline 7 & 5 & 1000 & $\$ 64,882$ & $\$ 78,124$ & $\$ 71,318$ & $\$ 71,567$ & $\$ 74,148$ & $\$ 76,409$ \\
\hline 7 & 6 & 1000 & $\$ 62,234$ & $\$ 78,124$ & $\$ 71,318$ & $\$ 71,567$ & $\$ 74,148$ & $\$ 76,409$ \\
\hline 8 & 2 & 1000 & $\$ 74,074$ & $\$ 87,409$ & $\$ 80,979$ & $\$ 81,821$ & $\$ 84,526$ & $\$ 89,441$ \\
\hline 8 & 3 & 1000 & $\$ 73,992$ & $\$ 87,409$ & $\$ 80,979$ & $\$ 81,821$ & $\$ 84,526$ & $\$ 89,441$ \\
\hline 8 & 4 & 1000 & $\$ 73,158$ & $\$ 87,409$ & $\$ 80,979$ & $\$ 81,821$ & $\$ 84,526$ & $\$ 89,441$ \\
\hline 8 & 5 & 1000 & $\$ 66,805$ & $\$ 87,409$ & $\$ 80,979$ & $\$ 81,821$ & $\$ 84,526$ & $\$ 89,441$ \\
\hline 8 & 6 & 1000 & $\$ 66,752$ & $\$ 87,409$ & $\$ 80,979$ & $\$ 81,821$ & $\$ 84,526$ & $\$ 89,441$ \\
\hline 9 & 2 & 100 & $\$ 87,015$ & $\$ 97,395$ & $\$ 90,870$ & $\$ 91,310$ & $\$ 95,892$ & $\$ 100,354$ \\
\hline 9 & 3 & 100 & $\$ 76,273$ & $\$ 97,395$ & $\$ 90,870$ & $\$ 91,310$ & $\$ 95,892$ & $\$ 100,354$ \\
\hline 9 & 4 & 100 & $\$ 75,829$ & $\$ 97,395$ & $\$ 90,870$ & $\$ 91,310$ & $\$ 95,892$ & $\$ 100,354$ \\
\hline 9 & 5 & 100 & $\$ 75,337$ & $\$ 97,395$ & $\$ 90,870$ & $\$ 91,310$ & $\$ 95,892$ & $\$ 100,354$ \\
\hline 9 & 6 & 100 & $\$ 75,625$ & $\$ 97,395$ & $\$ 90,870$ & $\$ 91,310$ & $\$ 95,892$ & $\$ 100,354$ \\
\hline 10 & 2 & 100 & $\$ 89,689$ & $\$ 109,346$ & $\$ 100,499$ & $\$ 100,553$ & $\$ 104,636$ & $\$ 109,009$ \\
\hline 10 & 3 & 100 & $\$ 82,507$ & $\$ 109,346$ & $\$ 100,499$ & $\$ 100,553$ & $\$ 104,636$ & $\$ 109,009$ \\
\hline 10 & 4 & 100 & $\$ 81,574$ & $\$ 109,346$ & $\$ 100,499$ & $\$ 100,553$ & $\$ 104,636$ & $\$ 109,009$ \\
\hline 10 & 5 & 100 & $\$ 81,683$ & $\$ 109,346$ & $\$ 100,499$ & $\$ 100,553$ & $\$ 104,636$ & $\$ 109,009$ \\
\hline 10 & 6 & 100 & $\$ 82,041$ & $\$ 109,346$ & $\$ 100,499$ & $\$ 100,553$ & $\$ 104,636$ & $\$ 109,009$ \\
\hline
\end{tabular}

As it is shown in Table 4.9, in all the experiments the performance of proposed approximation approach (extended Extreme-states method) is better than Baseline policy. The extended Extreme-states approximation approach has almost 20\% lower cost than Baseline policy. 


\subsection{Conclusions}

In this chapter a dynamic programming model to determine the suppliers' inspection strategy in a supply chain is proposed. A multi-period system was considered where there was a capacity limit for inspection. The objective was to minimize the inspection cost, the expected corrective action cost, and the expected missed detection cost simultaneously.

The structural properties of the proposed DP model for a single supplier were examined. The properties of the single supplier were incorporated to multi suppliers problem to develop an effective approximate DP algorithm to find high-quality solutions. The numerical experiments indicate that the proposed model entails a lower cost than the FDA's current inspection policy. 


\section{CHAPTER 5. SUMMARY AND CONCLUDING REMARKS}

This dissertation, examines how supply chains should be designed to mitigate the effects of supplier failures. It is explored how integrating inspection policy decisions into facility location and supply chain design can impact the solutions. In addressing the risk of supplier unreliability in the supply chain, three optimization models and algorithms were proposed. In Chapter 2 and Chapter 3 of this dissertation, a supply chain design that is both reliable and cost-efficient is sought. In Chapter 4, the operational inspection decision for a given supply chain with multiple unreliable supply sources is examined.

Further, in chapter 2, a comprehensive two-stage stochastic programming model to design a robust supply chain is developed. Unreliable supplier sourcing was considered in two-tiered with correlated failures, and the option to inspect. A method based on improved Integer L-shaped method was used to solve the model. This model was exercised to provide insights about how key parameters (capacity, correlation, inspection) impact on the obtained solutions and the expected total cost of solutions. This dissertation naturally leads to a number future research extensions. In this dissertation, it was assumed that when tainted material is detected, it is discarded. In the motivating application, this must occur as pharmaceutical and food products cannot typically be reworked or fixed once discovered. However, for other types of products such as electronics, faulty material can be reworked to return it to working order prior to shipping it to a customer. This can be considered in future research. As explained, the solution methodology used for this problem is not tailored solve the large-scale instances of problem. This is an area of future research that should be considered. Another direction for future research is to examine the effect of disruption on connectivity in the network. 
In addition, in Chapter 3, the single objective version of the problem is extended to a multi-objective stochastic model to explore the tradeoffs between costs and risk. Also, two types of disruption (quality and availability) have been considered in the model. A hybrid genetic algorithm is then developed to solve the multi-objective stochastic model. In this algorithm, data envelopment analysis was used to calculate the fitness value of supply chain configurations. The hybrid approach removes dominating individuals and yields to desirable efficient frontiers faster than methods based on only genetic algorithm. A number of future research extensions of the current study may be conducted. First, in this dissertation, a supply chain design that is robust against supply side disruption is sought. However, another important point is demand uncertainty and its effect on supplier selection and risk. Second, several different DEA models exist in the literature and may be implemented. Nevertheless, the numerical experiments indicated that most of them are not effective enough to distinguish truly efficient design points from inefficient ones. Also, in this dissertation one input and two outputs for the DEA were considered. However, it is believed that the performance of the proposed solution approach would be much better in case there are more number of inputs and/or outputs in the DEA.

Finally, in Chapter 4, operational inspection decisions were investigated in multiperiod supply chain with multiple unreliable supply sources. A dynamic programming (DP) model is performed to minimize the total inspection and corrective costs as well as the cost of disruption caused by missed detection in the supply chain. Also, an approximate DP algorithm is developed to identify high-quality solutions. The current FDA inspection policy was evaluated; numerical experiments indicated that proposed DP models result in significantly lower costs than the FDA's current inspection policy. This dissertation leads to a number of future research possibilities. The proposed approximate DP may be improved by incorporating approaches based on the constraints generation. In addition, in the numerical experiments, the performance of 
proposed approximate DP algorithm with Baseline policy were compared. Also, it was assumed that Baseline policy requires the inspection of suppliers every $t$ periods. However, it is possible that suppliers have different $t$ considering their characteristics. Thus, there is a need to evaluate and compare these types of policies with proposed approximate DP algorithm to better understand the performance of approximate DP algorithm.

Overall, the findings from this dissertation can help practitioners while designing supply chains, as well as helping policymakers to understand the impact of different disruption mitigation strategies on cost and risk in the supply chain. 


\section{BIBLIOGRAPHY}

[1] P. Chang, A. Bayhaqi, and B. Z. Yuhua. Concepts and trends in global supply, global value and global production chains. Technical Report 1, Asia-Pacific Economic Cooperation Policy Support Unit, May 2012.

[2] P. P. Singh. Japan supply chain break down to hurt global production, bbc news. http://www.bbc.co.uk/news/12858580, March 252011.

[3] N. Bunkley. Japan's automakers expect more delays, the new york times. http://www.nytimes.com/2011/03/19/business/global/19auto.html?_r=1, March 182011.

[4] G. Harris. U.s. identifies tainted heparin in 11 countries, April 2008. URL http: //www. nytimes. com/2008/04/22/health/22iht-22fda.12210515.html.

[5] H. Gardiner. U.s. identfies tainted heparin in 11 countries. The New York Times, April 22 2008. URL http://www.nytimes.com/2008/04/22/health/policy/ $22 \mathrm{fda} . \mathrm{html}$.

[6] When the chain breaks: being too lean and mean is a dangerous thing, June 2006. URL http://www. economist. com/node/7032258.

[7] S. Lohr. Stress test for the global supply chain, March 2011. URL http://www . nytimes . com/2011/03/20/business/20supply.html?_r=1\&pagewanted=all.

[8] Japan and the global supply chain: Broken links, March 2011. URL http: //www . economist. com/node/18486015.

[9] E. coli death toll rises to 31; sprouts traced to trash in home, June 2011. URL http://www. cnn.com/2011/WORLD/europe/06/10/europe.e.coli/ index.html?hpt=hp_bn6. 
[10] Egg contamination and recalls, September 2010. URL http://topics .nytimes . com/top/reference/timestopics/subjects/e/eggs/contamination_and_ recalls/index.html.

[11] B. Wassener. Chinese heparin supplier posts strong trading debut, May 2010. URL http://www. nytimes. com/2010/05/07/business/global/07drug.html.

[12] L. V. Snyder. Facility location under uncertainty: A review. IIE Transactions, 38:547-564, 2006.

[13] J. Wechsler. Fda's inspection program under scrutiny: The heparin safety crisis puts a spotlight on manufacturing processes and regulatory oversight. BioPharm International, 21(4), 2008.

[14] J. C. David, D. D. Yao, and S. Zheng. Optimal replenishment and rework with multiple unreliable supply sources. Operations Research, 49(3):430-443, 2001.

[15] M. Hariga and N. Azaiez. Heuristic procedures for the single facility inspection problem with minimal repair and increasing failure rate. The Journal of the Operational Research Society, 57(9):1081-1088, 2006.

[16] Y. H. Chun. Bayesian inspection model for the production process subject to a random failure. IIE Transactions, 42(4):304-316, 2010.

[17] P. D. Berger, A. Gerstenfeld, and A. Z. Zeng. How many suppliers are best? a decision-analysis approach. Omega, 32(1):9-15, 2004.

[18] A. J. Ruiz-Torres and F. Mahmoodi. The optimal number of suppliers considering the costs of individual supplier failures. Omega, 35(1):104-115, 2007.

[19] T. Sawik. Selection of supply portfolio under disruption risks. Omega, 39(2): 194-208, 2011. 
[20] R. Gaonkar and N. Viswanadham. A conceptual and analytical framework for the management of risk in supply chains. In IEEE International Conference on Robotics and Automation, volume 3, pages 2699-2704, 2004.

[21] E. Iakovou, D. Vlachos, and A. Xanthopoulos. An analytical methodological framework for the optimal design of resilient supply chains. International Journal of Logistics Economics and Globalisation, 1(1):1-20, 2007.

[22] H. Frank. Optimum locations on a graph with probabilistic demands. Operations Research, 14(3):409-421, 1966.

[23] M. S. Daskin. Application of an expected covering model to emergency medical service system design. Decision Sciences, 13(3):416-439, 1982.

[24] M. S. Daskin. A maximum expected covering location model: formulation, properties and heuristic solution. Transportation Science, 17(1):48-70, 1983.

[25] M. O. Ball and F. L. Lin. A reliability model applied to emergency service vehicle location. Operations Research, 41:18-36, 1993.

[26] C. Revelle and K. Hogan. The maximum availability location problem. Transportation Science, 23(3):192-200, 1989.

[27] M. Y. Carson and R. Batta. Locating an ambulance on the amherst campus of the state university of new york at buffalo. Interfaces, 20(5):43-49, 1990.

[28] L. V. Snyder and M. S. Daskin. Reliability models for facility location: The expected failure cost case. Transportation Science, 39(3):400-416, 2005.

[29] T. Cui, Y. Ouyang, and Z. M. Shen. Reliable facility location design under the tisk of disruptions. Operations Research, 58:998-1011, 2010.

[30] D. Gade and E. A. Pohl. Sample average approximation applied to the capacitated facilities location problem with unreliable facilities. Proceedings of the 
Institution of Mechanical Engineers, Journal of Risk and Reliability, 223(4):259$269,2009$.

[31] P. Peng, L. V. Snyder, A. Lim, and Z. Liu. Reliable logistics networks design with facility disruptions. Transportation Research Part B: Methodological, 45(8): 1190-1211, 2011.

[32] S. Lee. On solving unreliable planar location problems. Computers $\mathscr{E}$ Operations Research, 28(4):329-344, 2001.

[33] M. Bundschuh, D. Klabjan, and D. Thurston. Modeling robust and reliable supply chains. Optimization, Online e-Print:1-32, 2003.

[34] C. K. Y. Lin and R. C. W. Kwok. Multi-objective metaheuristics for a locationrouting problem with multiple use of vehicles on real data and simulated data. European Journal of Operational Research, 175(3):1833-1849, 2006.

[35] R. Caballero, M. Gonzalez, F. M. Guerrero, J. Molina, and C. Paralera. Solving a multiobjective location routing problem with a metaheuristic based on tabu search. application to a real case in andalusia. European Journal of Operational Research, 177(3):1751-1763, 2007.

[36] H. Yildiz, M. P. Johnson, and S. Roehrig. Planning for meals on wheels: Algorithms and application. Journal of Operational Research, 2012.

[37] H. Yildiz, R. Ravi, and W. Fairey. Integrated optimization of customer and supplier logistics at robert bosch llc. European Journal of Operational Research, 207(1):456-464, 2010.

[38] S. J. Erlebacher and R. D. Meller. The interaction of location and inventory in designing distribution systems. IIE Transactions, 32(2):155-166, 2000. 
[39] M. S. Daskin, C. R. Coullard, and Z. M. Shen. An inventory-location model: formulation, solution algorithm and computational results. Annals of Operations Research, 110(1):83-106, 2002.

[40] H. Min, V. Jayaraman, and R. Srivastava. Combined location-routing problems: A synthesis and future research directions. European Journal of Operational Research, 108(1):1-15, 1998.

[41] G. Nagy and S. Salhi. Location-routing: Issues, models and methods. European Journal of Operational Research, 177(2):649-672, 2007.

[42] X. Li and Y. Ouyang. A continuum approximation approach to reliable facility location design under correlated probabilistic disruptions. Transportation Research Part B, 44(4):535-548, 2010. ISSN 0191-2615.

[43] F. Liberatore, M. P. Scaparra, and M. S. Daskin. Hedging against disruptions with ripple effects in location analysis. Omega, 40(1):21-30, 2012. ISSN 03050483.

[44] R. Klimberg, C. Revelle, and J. Cohon. Improving the effectiveness of fda drug inspection. Operations research, 40(5):845-855, 1992.

[45] B. F. Qaqish. A family of multivariate binary distributions for simulating correlated binary variables with specified marginal means and correlations. Biometrika, 90(2):455-463, 2003.

[46] G. Laporte and F. V. Louveaux. The integer l-shaped method for stochastic integer programs with complete recourse. Operations Research Letters, 13(3): 133-142, 1993.

[47] R. M. V. Slyke and R. Wets. L-shaped linear programs with applications to 
optimal control and stochastic programming. SIAM Journal on Applied Mathematics, 17(4):638-663, 1969.

[48] S. Sen, J. Higle, and L. Ntaimo. A summary and illustration of disjunctive decomposition with set convexification. In Network Interdiction and Stochastic Integer Programming, volume 22, pages 105-125. Springer, 2003.

[49] S. Sen and J. L. Higle. The c3 theorem and a d2 algorithm for large scale stochastic mixed-integer programming: Set convexification. Mathematical Programming, 104(1):1-20, 2005.

[50] L. Ntaimo and M. W. Tanner. Computations with disjunctive cuts for two-stage stochastic mixed 0-1 integer programs. Journal of Global Optimization, 41(3): 365-384, 2008.

[51] L. Ntaimo. Disjunctive decomposition for two-stage stochastic mixed-binary programs with random recourse. Operations Research, 58(1):229-243, 2010.

[52] L. Ntaimo and S. Sen. A comparative study of decomposition algorithms for stochastic combinatorial optimization. Computational Optimization and Applications, 40(3):299-319, 2008.

[53] L. V. Snyder and M. S. Daskin. Models for reliable supply chain network design. In Reliability and Vulnerability in Critical Infrastructure: A Quantitative Geographic Perspective. Forthcoming, pages 257-289. Springer, 2006.

[54] E. O. Afoakwa. Melamine contamination of infant formula in china: The causes, food safety issues and public health implications. African Journal of Food, Agriculture, Nutrition and Development, 8(4), 2008.

[55] Courts compound pain of chinas tainted milk, October 162008. 
URL http://www.nytimes.com/2008/10/17/world/asia/17milk.html? pagewanted=all\&_r=0.

[56] Tainted milk scandal impacts over three dozen countries, October 20 2008. URL http://worldfocus.org/blog/2008/10/20/ tainted-milk-scandal-impacts-over-three-dozen-countries/2020/.

[57] D. J. Rosenkrantz, G. K. Tayi, and S. S. Ravi. Algorithms for path-based placement of inspection stations on networks. Journal on Computing, 12(2):136-149, 2000.

[58] A. Azaron, K. N. Brown, S. A. Tarim, and M. Modarres. A multi-objective stochastic programming approach for supply chain design considering risk. Int. J. Production Economics, 116:129-138, 2008.

[59] G. Guillen, F. D. Mele, M. J. Bagajewicz, A. Espuna, and L. Puigjaner. Multiobjective supply chain design under uncertainty. Chemical Engineering Science, 60:1535-1553, 2005.

[60] Z. Liu and A. Nagurney. Supply chain outsourcing under exchange rate risk and competition. Omega, 39:539549, 2011.

[61] R. B. Franca, E. C. Jones, C. N. Richards, and J. P. Carlson. Multi-objective stochastic supply chain modeling to evaluate tradeoffs between profit and quality. Int J. Production Economics, 127:292-299, 2010.

[62] E. H. Sabri and B. M. Beamon. A multi-objective approach to simultaneous strategic and operational planning in supply chain design. Omega, 28:581-598, 2000.

[63] T. C. Matisziw, A. T. Murray, and T. H. Grubesic. Strategic network restoration. Networks and Spatial Economics, 10(3):345-361, 2010. 
[64] L. V. Snyder, M. P. Scaparra, M. S. Daskin, and R. L. Church. Planning for disruptions in supply chain networks. In TutORials in Operations Research. INFORMS, pages 234-257, 2006.

[65] L. Qi, Z. M. Shen, and L. V. Snyder. A continuous-review inventory model with disruptions at both supplier and retailer. Production and Operations Management, 18(5):516-532, 2009.

[66] L. Qi, Z. M. Shen, and L. V. Snyder. The effect of supply disruptions on supply chain design decisions. Transportation Science, 44(2):274-289, 2010.

[67] T. C. Matisziw, T. H. Grubesic, and J. Guo. Robustness elasticity in complex networks. PLoS ONE, 7(7):e39788, 2012.

[68] V. Serrano, M. Alvarado, and C. A. Coello Coello. Optimization to manage supply chain disruptions using the nsga-ii. In Theoretical Advances and Applications of Fuzzy Logic and Soft Computing, volume 42 of Advances in Intelligent and Soft Computing, pages 476-485, 2007.

[69] L. Bianchi, M. Dorigo, L. M. Gambardella, and W. J. Gutjahr. A survey on metaheuristics for stochastic combinatorial optimization. Natural Computing, 8 (2):239-287, 2009 .

[70] D. E. Goldberg. Genetic algorithms in search, optimization and machine learning. Addison-Wesley Longman, Inc., New York, 1989.

[71] W. W. Cooper, L. M. Seiford, and J. Zhu. Handbook on Data Envelopment Analysis, chapter Data Envelopment Analysis: History, Models and Interpretations. Taylor \& Francis US, 2004.

[72] L. M. Seiford and J. Zhu. Modeling undesirable factors in efficiency evaluation. European Journal of Operational Research, 142:16-20, 2002. 
[73] A. Hadi-Vencheh, R. Kazemi-Matin, and M. Tavassoli-Kajani. Undesirable factors in efficiency measurement. Applied Mathematics and Computation, 163: $547-552,2005$.

[74] Y. Bian. Efficiency evaluation with undesirable factors based on dea. In Wireless Communications, Networking and Mobile Computing, 2008. WiCOM '08. 4th International Conference on, pages 1-5, oct. 2008. doi: 10.1109/WiCom.2008. 1270.

[75] Y. B. Yun, H. Nakayama, T. Tanino, and M. Arakawa. Generation of efficient frontiers in multi-objective optimization problems by generalized data envelopment analysis. European Journal of Operational Research, 129:586-595, 2001.

[76] G. Whittaker, R. Confesor Jr, S. M. Griffith, R. Fare, S. Grosskopf, J. J. Steiner, G. W. Mueller-Warrant, and G. M. Banowetz. A hybrid genetic algorithm for multiobjective problems with activity analysis-based local search. European Journal of Operational Research, 193:195-203, 2009.

[77] Z. Li, H. Liao, and D. W. Coit. A two-stage approach for multi-objective decision making with applications to system reliability optimization. Reliability Engineering and System Safety, 94:1585-1592, 2009.

[78] D. T. Wang, L. F. Ochoa, and G. P. Harrison. Modified ga and data envelopment analysis for multistage distribution network expansion planning under uncertainty. IEEE Transactions on Power Systems, 26(2):897-904, 2011.

[79] R. C. Lin, M. Y. Sir, and K. S. Pasupathy. Multi-objective simulation optimization using data envelopment analysis and genetic algorithm: Specific application to determining optimal resource levels in surgical services. Omega, 41(5):881-892, 2013. 
[80] R. Fare and S. Grosskopf. Modeling undesirable factors in efficiency evaluation: Comment. European Journal of Operational Research, 157:242-245, 2004.

[81] Bridge inspection, posting, and responsibility, August 2011. URL http://www. maine.gov/mdot/publicbridges/bipr.htm.

[82] G. Rabinowitz and H. Emmons. Optimal and heuristic inspection schedules for multistage production systems. IIE Transactions, 29(12):1063-1071, 1997.

[83] D. D. Yao and S. Zheng. Sequential inspection under capacity constraints. Operations Research, 47(3):410-421, 1999.

[84] Y. R. Shiau. Inspection resource assignment in a multistage manufacturing system with an inspection error model. Journal of Production Research, 40(8): 1787-1806, 2002.

[85] K. Kogan and T. Raz. Optimal allocation of inspection effort over a finite planning horizon. IIE Transactions, 34(6):515-527, 2002.

[86] H. Emmons and G. Rabinowitz. Inspection allocation for multistage deteriorating production systems. IIE Transactions, 34(12):1031-1041, 2002.

[87] S. H. Sheu, Y. C. Chen, W. Y. Wang, and N. H. Shin. Economic optimization of off-line inspection with inspection errors. The Journal of the Operational Research Society, 54(8):888-895, 2003.

[88] W. Jang and J. G. Shanthikumar. Sequential process control under capacity constraints. European Journal of Operational Research, 155:695-714, 2004.

[89] S. Anily and A. Grosfeld-Nir. An optimal lot-sizing and offline inspection policy in the case of nonrigid demand. Operations Research, 54(2):311-323, 2006. 
[90] C. H. Wang, N. H. Shih, and W. C. Tsai. Utilizing the information theory of entropy to solve an off-line inspection problem. 4OR: A Quarterly Journal of Operations Research, 9(4):391-401, 2011.

[91] T. McClurg and S. Chand. A parallel machine replacement model. Naval Research Logistics, 49(3):275-287, 2002.

[92] S. Childress and P. Durango-Cohen. On parallel machine replacement problems with general replacement cost functions and stochastic deterioration. Naval Research Logistics, 52(5):409-419, 2005.

[93] V. M. Guillaumot, P. L. Durango-Cohen, and S. M. Madanat. Adaptive optimization of infrastructure maintenance and inspection decisions under performance model uncertainty. Journal of Infrastructure Systems, 9(4):133-139, 2003.

[94] S. Ozekici and S. R. Pliska. Optimal scheduling of inspections: A delayed markov model with false positives and negatives. Operations Research, 39(2):261-273, 1991.

[95] A. G. Shetwan, V. I. Vitanov, and B. Tjahjono. Allocation of quality control stations in multistage manufacturing systems. Computers $\& \mathcal{G}$ Industrial Engineering, 60(4):473-484, 2011.

[96] F. d'Epenoux. A probabilistic production and inventory problem. Management Science, 10(1):98-108, 1963.

[97] D. P. De Farias and B. Van Roy. The linear programming approach to approximate dynamic programming. Operations Research, 51(6):850-865, 2003.

[98] A. S. Manne. Linear programming and sequential decisions. Management Science, 6(3):259-267, 1960. 


\section{VITA}

Mahmood Pariazar completed his PhD in Industrial and Manufacturing Systems Engineering at the University of Missouri-Columbia. His focus areas are in dynamic and stochastic programming, large-scale optimization, and network design, with the emphasis on their applications in industrial projects. He completed his master's degree in Industrial Engineering department at Amirkabir University of Technology in 2007, Tehran, Iran. He received his bachelor's degree in Industrial Engineering

department at K.N.Toosi University of Technology in 2005, Tehran, Iran. He has also worked with companies such as MoDOT, and $\mathrm{CNH}$ on supply chain improvement projects. 